\title{
A micromechanics-based non-local damage to crack transition framework for porous elastoplastic solids
}

\author{
Julien Leclerc ${ }^{\mathrm{a}}$, Van-Dung Nguyen ${ }^{\mathrm{a}}$, Thomas Pardoen ${ }^{\mathrm{b}}$, Ludovic Noels ${ }^{\mathrm{a}}$, \\ ${ }^{a}$ Computational $\& 3$ Multiscale Mechanics of Materials, University of Liège, Belgium \\ ${ }^{b}$ Institute of Mechanics, Materials and Civil Engineering, UCLouvain, Belgium
}

\begin{abstract}
The failure process of ductile porous materials is simulated by representing the damage nucleation, growth and coalescence stages up to crack initiation and propagation using a physically-based constitutive model. In particular, a non-local damage to crack transition framework is developed to predict the fracture under various loading conditions while minimising case-dependent calibration process. The formulation is based on a discontinuous Galerkin method, making it computationally efficient and scalable. The initial stable damage process is simulated using an implicit non-local damage model ensuring solution uniqueness beyond the onset of softening relying on the Gurson-Tvergaard-Needleman (GTN) model. Once the coalescence criterion is satisfied, which can physically arise before or during the softening stage, a cohesive band is introduced. Within the cohesive band, a void coalescencebased governing law is solved, accounting for the stress triaxiality state and material history, in order to capture the near crack tip failure process in a micromechanically sound way. Two coalescence models are then successively considered and compared. First, with a view to model verification towards literature results, a numerical coalescence model detects crack initiation at loss of ellipticity of a local model, and the crack opening is governed by ad-hoc parameters of the GTN model. Alternatively, the Thomason criterion is used to detect crack nucleation during the softening stage while the Thomason coalescence model governs the crack opening process. This latter model is able to reproduce slant and cup-cone failure modes in plane-strain and axisymmetric specimens, respectively.
\end{abstract}

Keywords: Ductile fracture, Cohesive band model, Damage to crack transition, Discontinuous Galerkin, Porous plasticity

\section{Introduction and motivations}

Accurate numerical predictions of ductile failure under complex loading conditions involve many yet unsolved challenges. The ductile damage process usually starts with the nucleation of voids or microcracks which add to the pre-existing porosity resulting from

Email address: 1.noels ulg.ac.be (Ludovic Noels) 
processing. The porosity then grows by plastic deformation. The process ends with strain and damage localisation due to shear banding or to microscopic void coalescence (Tekoğlu et al., 2015). The coalescence stage leads to the initiation and propagation of a macroscopic crack, and ultimately to material failure.

Several approaches have been developed to model the fracture process. The damage mechanisms can be represented by the continuous evolution of internal or damage variables. The continuous damage models (CDM) allow the description of a large range of degradation processes using a classical finite element (FE) formulation. The simplest approaches consist in using the progressive accumulation of damage to determine when failure occurs (Johnson and Cook, 1985, e.g.). In phenomenological CDM approaches, the damage growth induces the material properties degradation until failure (Lemaitre, 1986). The material degradation can also be represented by a micromechanics-based model, which follows the microstructure changes (Gurson, 1977). Among this last category, the Gurson (1977) model family has been developed since the seventies to take into account in a physically motivated way the microscopic porosity evolution in metals, see the complete reviews by Pineau et al. (2016); Benzerga et al. (2016); Besson (2010); Pardoen et al. (2010). The model derivation implies an associated plastic flow and also gives an evolution law for the void volume fraction parameter $f_{\mathrm{V}}$. The model was modified over the years to improve the accuracy of void growth predictions and to represent coalescence and final fracture using phenomenological arguments, as in the so-called Gurson-Tvergaard-Needleman (GTN) model (Tvergaard and Needleman, 1984). In particular, an effective porosity was introduced in the yield surface near fracture to generate realistic ductilities (Tvergaard and Needleman, 1984).

However, as shown by physical observations and by FE cell simulations by Koplik and Needleman (1988), the coalescence is more than just an abrupt void growth acceleration: the latter is rather a consequence of the former. Indeed, after a relatively diffuse plasticity and damage development stage, material behaviour suddenly changes into a localised deformation state. Plastic flow also shifts direction. All these changes indicate the onset of a new deformation mechanism. Therefore, even if a proper calibration of the GTN model can crudely reproduce the coalescence stage, a different model is more suitable to describe and to predict this physical process (Brocks et al., 1995; Benzerga and Leblond, 2010; Besson, 2010). In this context, different coalescence modes compete depending on the loading conditions and the micromechanical structure as recently studied by Pineau et al. (2016); Cortese et al. (2016); Liu et al. (2016); Brünig et al. (2018); Roth et al. (2018). The most investigated mode is by internal necking: the onset of coalescence is reached when plastic flow localises inside the intervoid ligament oriented normal to the main loading direction. The model of Thomason (1985a,b) provides a criterion based on the plastic limit-load of the intervoid ligament under tensile-dominated stress. This approach was then completed with evolution laws for the geometrical parameters of voids by Pardoen and Hutchinson (2000) and Benzerga (2002), and a fully analytical expression of the criterion was derived by Benzerga and Leblond (2014). Furthermore, by rewriting the criterion for arbitrary orientation, Pardoen and Hutchinson (2000) and Benzerga et al. (2004) derived a yield surface for the coalescence stage to be used in combination to a void growth surface. However, shear stress effects are not accounted for by the Thomason model. To address this issue, Tekoğlu et al. 
(2012) and Torki et al. (2017) have extended the Thomason approach combining shear and tensile loading. Reddi et al. (2019) have obtained similar results by using the multi-surface yield criterion developed by Keralavarma and Chockalingam (2016); Keralavarma (2017). Nguyen et al. (Submitted) have also introduced a multi-surface approach accounting for void growth, internal necking and shearing mechanisms in a non-local context to avoid loss of solution uniqueness. In addition, Torki (2019) unified both void growth and coalescence criteria under tensile and shear conditions in a unique but complex yield function. Besides, Leblond and Mottet (2008) have provided an approximate solution of the problem by substituting the central voided region of the RVE by a sandwich made of von Mises and porous layers.

However, whatever the considered (local) continuous damage models, they are intrinsically mesh-dependent during the softening regime (Bažant et al., 1984; Peerlings et al., 1996; Reusch et al., 2003b). Indeed, at this point, governing equations loose their ellipticity. Damage tends then to spuriously localise in a vanishingly thick volume. Regularisation methods are therefore needed to overcome this issue like phase field approaches (Miehe et al., 2016a,b; de Borst and Verhoosel, 2016; Shen et al., 2019; Aldakheel et al., 2018) or non-local models (Peerlings et al., 2001; Nguyen et al., 2015). The phase field approach consists in replacing crack surface by a regularised or diffusive crack field while non-local models give up the local action principle and substitute some local internal variables by their non-local averaged counterparts. A similar framework can be recovered by the means of a micromorphic approach (Forest, 2009; Aldakheel, 2017).

Another approach to predict failure is to materialise all the damage process itself by crack propagation. One of the most popular methods is the cohesive zone model (CZM), in which a traction-separation law (TSL) determines the progressive loss of load-carrying capacity during the crack opening (Hillerborg et al., 1976). If the crack path is not a priori known, such discontinuities can be inserted through the elements by mesh enrichment (XFEM) (Moës et al., 1999, e.g.) or by element enrichment (EFEM) (Linder and Armero, 2007, e.g.). Both crack insertion methods generate arbitrary crack path through the FE mesh, once a crack direction criterion is provided, which is not a trivial task in 3D. However, they require important modifications in the finite element formulation, contrarily to the use of cohesive interface elements that are inserted between volume elements. These cohesive interface elements can be inserted during the simulation, for example by node splitting or re-meshing as done by Camacho and Ortiz (1996). In this case, the cohesive model is qualified as extrinsic. Unfortunately, these modifications of the mesh topology limit the method scalability, unless a graph-based internal structure is used (Mota et al., 2008; Paulino et al., 2008). Conversely, these elements can be intrinsically present at the beginning of the simulation. In this case, a (high) initial cohesive loading stiffness is necessary. However, this introduces mesh dependency and results in an inconsistent scheme which is only practical for a priori known crack paths (Xu and $\mathrm{Lu}, 2013)$. To overcome these problems, a scalable and consistent method is to introduce extrinsic cohesive zones through a discontinuous Galerkin (DG) framework (Mergheim et al., 2004; Radovitzky et al., 2011; Prechtel et al., 2011; Becker and Noels, 2012; Wu et al., 2014). It is based on the concept of weakly ensuring interelement continuity by adding consistency and penalty terms. Therefore, as discontinuities 
are inherently present between elements in the DG formulation, the DG-constraints are released at crack initiation and replaced by the cohesive relationship. By this way, a large number of cracks can be inserted and the propagation can be modelled without scalability or consistency issues as demonstrated by Radovitzky et al. (2011).

In the literature, the fracture mechanics and the continuous damage mechanics are used to simulate ductile fracture. However, despite the capability of the CZM to introduce discontinuities and crack surfaces, it concentrates all the damage process at the crack tip and is unable to accurately represent the first stage of homogeneously distributed damage process. Using only continuous damage models entails highly distorted elements in highly damaged zones around the real crack surface, hurting numerical convergence (Mediavilla et al., 2005). Moreover, non-local damage models, used to eliminate mesh-dependency of their local counterparts, induce spurious damage extension in the last failure stage: damage artificially spreads out from the fictive crack path due to intense and concentrated straining (Geers et al., 1998). This effect can be limited by regularisation techniques as by choosing wisely the diffused quantity, using a variable non-local length (Geers et al., 1998; Poh and Sun, 2016; Vandoren and Simone, 2018) or by phase field approaches. However, this does not solve the problem of highly distorted elements.

Neither continuous nor discontinuous approaches are able to describe alone the whole process, which has motivated the development of non-local damage models with a crack transition to take advantage of the complementarity of the CDM and CZM approaches (Wu et al., 2014). In a such CDM/CZM framework, a non-local CDM simulates the initial homogeneous damage stage and its localisation. Then, the crack propagation is modelled using a CZM until complete failure, and can be inserted with any appropriate crack insertion technique, such as remeshing (Cuvilliez et al., 2012), XFEM (Wang and Waisman, 2018), EFEM, DG (Wu et al., 2014; Aduloju and Truster, 2019), etc. This transition between non-local models and cohesive zone methods is possible since both approaches can be made theoretically equivalent. Indeed, as shown by numerous studies (Cazes et al., 2009, 2010; Wu et al., 2014; Leclerc et al., 2018, e.g.), the CDM/CZM transition framework is consistent if the total dissipated energy during the entire fracture process corresponds to the physicallybased one. Therefore, at its insertion, the CZM has to dissipate the amount of energy not yet dissipated in the damage process as represented by the non-local model. This energetic quantity can be determined from a reference case in terms of damage (Comi et al., 2006), or effective stress value (Wu et al., 2014). Scheyvaerts et al. (2010) have proposed a micromechanics-based model to predict this final failure energy and unloading ramp.

However, as pointed out by Wu et al. (2014), a classical CZM approach does not include the influence of in-plane stress components, and thus does not correctly represent pressure and triaxiality, or Lode variable effects. These latter effects are essential for accurate predictions of ductile failure unless the CZM is inserted at a very high level of damage near complete failure, which is not always realistic from a physical point of view. Therefore, in order to include these effects, a simple method is to define CZM key parameters directly dependent on stress triaxiality (Siegmund and Brocks, 2000). But the determination and the use of such a relationship rise the complex question of its calibration in terms of the material history. A more general and natural way is to redefine a $3 \mathrm{D}$ stress state at the interface 
and deduce from it the cohesive behaviour. All the degradation process after localisation is therefore assumed to take place inside a thin band around the crack surfaces. It can be computed through the coupling with an energetic interface (Esmaeili et al., 2017). A 3D state can be recovered through multi-scale analyses: in Bosco et al. (2015), the cohesive behaviour is extracted from a representative volume element. This damaging band can be of finite thickness and introduced by an embedded weak discontinuity through the elements (Huespe et al., 2009, 2012). This cohesive band can also be concentrated at the crack surfaces (Remmers et al., 2013) and introduced by an extrinsic cohesive zone model (Leclerc et al., 2018).

This latest method, so-called the cohesive band model (CBM), has the advantages of (i) requiring relatively few modifications of the cohesive model approach compared to the multi-scale or the finite band method; (ii) reusing the underlying damage model; and (iii) following on-the-fly the local stress state and material history. It only introduces one extra parameter: the cohesive band thickness $h_{\mathrm{b}}$ which controls the total dissipated energy. The effect of $h_{\mathrm{b}}$ has been studied by Leclerc et al. (2018) in the case of elasticity in a small strain setting. In particular, it has been shown that this fictitious thickness can be evaluated from the knowledge of the critical energy release rate $G_{\mathrm{c}}$.

Based on all these previous considerations, the objective of this paper is to develop a non-local damage to crack transition suitable to ductile failure. In particular, the framework should be able to predict the failure under various loading conditions while minimising case-dependent calibration. Emphasis is brought here on the methodology adapted to porous plasticity and its numerical aspects. The method is requested to be consistent, mesh-independent, scalable and computationally efficient, and applicable in 3D. Besides, the developed methodology should be versatile enough to account for different coalescence criteria and coalescence models in order to represent the crack initiation and propagation.

To achieve this goal, a damage to crack transition is developed for ductile materials. The framework includes an implicit non-local damage model (Peerlings et al., 1996; Reusch et al., 2003b,a) and a cohesive band model (Remmers et al., 2013) inside a DG-formulation (Radovitzky et al., 2011). Although such a damage to crack transition has been developed in a previous work in the context of small strain elastic-damage behaviour (Leclerc et al., 2018), the development of such a methodology for ductile materials requires to address several challenges since (i) the formulation should be stated in a finite-strain setting; (ii) the damage to crack transition should be driven by micro-mechanics-based consideration while it was purely phenomenological in the elastic-damage case; and (iii) the monolithic resolution of the combined non-local damage-cohesive band model requires the evaluation in a closed form of the weak formulation and material models tangent operators.

In this work, the initial diffuse damage state is simulated using an implicit non-local damage model without mesh-dependency beyond the onset of softening. This is of particular importance when considering a physically-based crack initiation criterion which can be met in the strain softening stage, as it will be shown in this paper. Once this crack insertion criterion is satisfied, an extrinsic cohesive band is inserted between volume elements using the discontinuous Galerkin framework, hence modelling crack initiation. The cohesive band model allows the determination on-the-fly of the cohesive behaviour while 
taking into account the current local 3D stress state and the material history. As the framework is intended to be applied to ductile elastoplastic solids such as most ductile metals or some polymers showing cavitations, a porous plastic model is chosen as constitutive material model (Besson, 2009). It includes the description of the void growth and coalescence steps, but also the nucleation model developed by Chu and Needleman (1980) and the shearinduced growth term proposed by Nahshon and Hutchinson (2008). Two coalescence models are then successively considered. On the one hand, with a view to the model verification with the analyses by Huespe et al. (2012), a numerical coalescence model is used to detect crack initiation at loss of ellipticity of a local model, whilst the crack opening is governed by an adequate calibration of the Gurson model parameters. On the other hand, following the work by Pardoen and Hutchinson (2000), a physically-based Thomason (1985a,b) criterion is used as crack insertion criterion, and the coalescence model is used as governing law of the CBM to model the crack opening.

The outline of the paper is as follows. The non-local porous elastoplastic model is first presented in Section 2. Afterwards, the damage to crack transition is introduced with the cohesive band model in Section 3. In particular, the questions of the crack insertion criterion and the cohesive band thickness are addressed. The implementation and monolithic resolution of the current framework is detailed in Section 4. The weak form is deduced from the strong form of equations and is then discretised following the DG-formulation. The numerical time integration is also discussed. Finally, Section 5 is devoted to illustrating the framework capability to correctly represent the ductile fracture process. In particular, the results obtained by a local form of the porous elastoplastic model and by introducing a crack at loss of ellipticity are shown to be in good agreement with the results by Huespe et al. (2012). Then, we show that when considering the non-local porous plasticity model combined to the Thomason criterion and the Thomason coalescence model to govern respectively the crack initiation and propagation, the framework is able to reproduce slant and cup-cone failure modes in plane-strain specimens and axisymmetric bars, smooth and notched ones, respectively. These complex failure patterns driven by shearing are herein captured, and by Huespe et al. (2012) as well, because of the shear stress state obtained by the free surface creation during crack propagation. Without the introduction of the crack, these patterns do not appear with a purely CDM model unless shear necking is considered in the yield surface as suggested by Reddi et al. (2019); Nguyen et al. (Submitted).

\section{Non-local micromechanics for porous plasticity}

In this section, the non-local continuum damage mechanics framework applied to porous plasticity is developed, combining the formulations of Reusch et al. (2003b,a) and Besson (2009). Cracks are ignored in this description and the body is assumed to be continuous as it is during the early damage stage. At first, the governing equations of the mechanical problem is provided. Then, a hyperelastic non-local porous plastic model is presented before being particularised to the considered deformation mechanisms. 


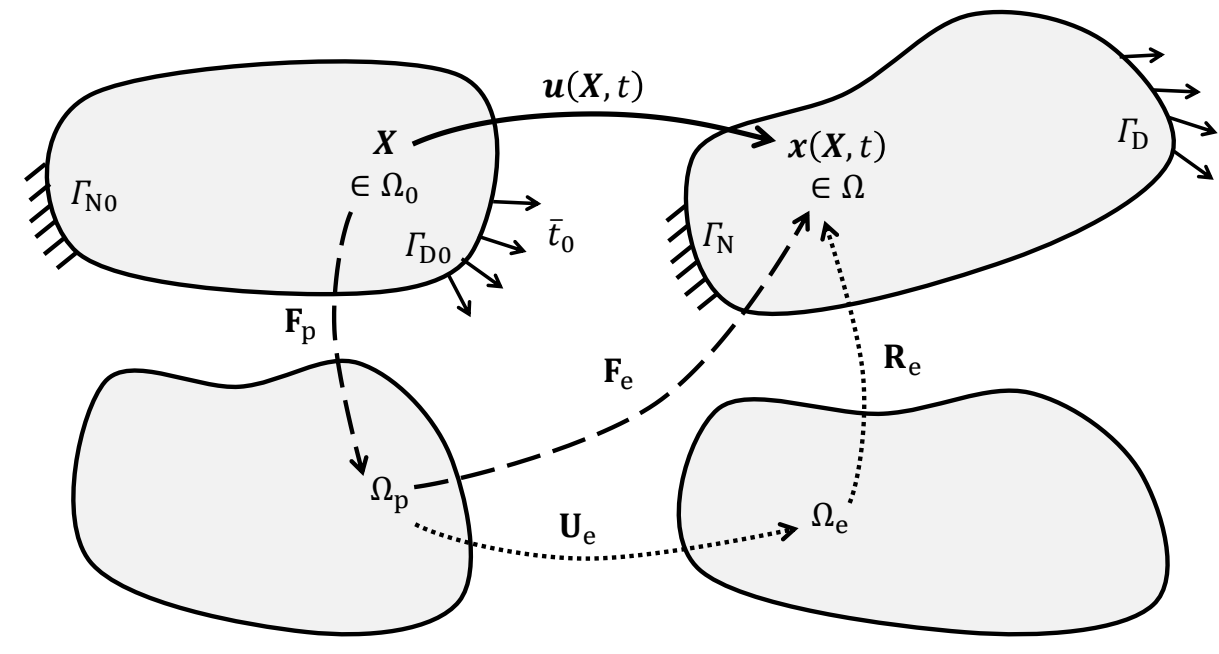

Figure 1: A material body in the reference configuration $\Omega_{0}$ (left) and in the current configuration $\Omega$ (right). The body deformation mapping is defined by $\boldsymbol{u}(\boldsymbol{X}, t)$ and the corresponding deformation gradient $\mathbf{F}$ between both configurations is decomposed into a plastic part $\mathbf{F}^{\mathrm{p}}$ followed by an elastic part $\mathbf{F}^{\mathrm{e}}$. The elastic part is also split in a symmetric stretch part $\mathbf{U}^{\mathrm{e}}$ and a rotational part $\mathbf{R}^{\mathrm{e}}$.

\subsection{Non-local problem statement}

Consider a material body $\Omega_{0} \subset \mathcal{R}^{3}$ in the reference configuration at time $t=0$ and its boundary surface $\Gamma_{0}$ as shown on Fig. 1 . The external surface $\Gamma_{0}$ is divided into a Dirichlet part $\Gamma_{\mathrm{D} 0}$ where displacements are prescribed, and a Neumann part $\Gamma_{\mathrm{N} 0}$ where the traction force is prescribed. This surface partition satisfies $\Gamma_{\mathrm{D} 0} \cap \Gamma_{\mathrm{N} 0}=\Gamma_{0}$ and $\Gamma_{\mathrm{D} 0} \cup \Gamma_{\mathrm{N} 0}=\emptyset$. $\Omega$ and $\Gamma$ denote respectively the counterparts of $\Omega_{0}$ and $\Gamma_{0}$ in the current configuration at a given time $t>0$. During this time evolution, the displacement of a material particle initially at the position $\boldsymbol{X} \in \Omega_{0}$ to the current spatial position $\boldsymbol{x}(\boldsymbol{X}, t) \in \Omega$ is defined by $\boldsymbol{u}(\boldsymbol{X}, t)=\boldsymbol{x}(\boldsymbol{X}, t)-\boldsymbol{X}$. The derivative of the current spatial position in terms of the material configuration, or in other words, the deformation gradient, is

$$
\mathbf{F}=\frac{\partial \boldsymbol{x}}{\partial \boldsymbol{X}}=\mathbf{I}+\frac{\partial \boldsymbol{u}}{\partial \boldsymbol{X}}
$$

with its Jacobian $J=\operatorname{det} \mathbf{F}>0$, and $\mathbf{I}$ the second-order identity tensor.

The linear momentum conservation, under large deformation setting over the body $\Omega$ with respect to the reference configuration, is given by

$$
\rho_{0} \ddot{\boldsymbol{u}}=\nabla_{0} \cdot \mathbf{P}^{\mathrm{T}}+\boldsymbol{b}_{0} \text { on } \Omega_{0},
$$

where $\rho_{0}$ is the initial mass density, $\nabla_{0}$ is the gradient operator with respect to the reference configuration, $\mathbf{P}$ the first Piola-Kirchhoff (PK1) stress tensor and $\boldsymbol{b}_{0}$ is the volumetric external force vector.

Boundary conditions are applied on the reference surface: prescribed displacement $\overline{\boldsymbol{u}}$ and traction surface $\overline{\boldsymbol{t}}_{0}$ are respectively applied on the Dirichlet boundary $\Gamma_{\mathrm{D} 0}$ and on the 
Neumann boundary $\Gamma_{\mathrm{N} 0}$ :

$$
\begin{array}{ll}
\boldsymbol{u}=\overline{\boldsymbol{u}} & \text { on } \Gamma_{\mathrm{D} 0}, \text { and } \\
\mathbf{P} \cdot \boldsymbol{N}=\overline{\boldsymbol{t}_{0}} & \text { on } \Gamma_{\mathrm{N} 0},
\end{array}
$$

with $\boldsymbol{N}$ the unit surface normal in the reference configuration and $\overline{\boldsymbol{t}_{0}}$ defined in the current configuration per unit surface in the reference configuration.

The boundary value problem defined by Eqs. (2) and (3) is completed by a material constitutive law which defines the evolution of the stress tensor $\mathbf{P}$ at time $t$ as

$$
\mathbf{P}(t)=\mathbf{P}\left(\mathbf{F}(t), \tilde{Z}(t) ; \boldsymbol{Z}\left(t^{\prime}\right), t^{\prime} \in[0, t]\right),
$$

in terms of the actual deformation gradient $\mathbf{F}$ and in terms of a set of local internal variables $\boldsymbol{Z}$ and, potentially, a set of non-local $\tilde{Z}$ internal variables representative of all the material history and path-dependence.

Non-local variables are introduced to avoid mesh-dependency. An implicit non-local gradient approach (Peerlings et al., 1996), equivalent to a micromorphic approach (Forest, 2009; Aldakheel, 2017), is preferred as it is truly non-local in contrast to an explicit form, as demonstrated by Peerlings et al. (2001). Moreover, the treatment of complex geometries and boundaries is here more simple and convenient than with an equivalent integral approach. Practically, some local variables $Z_{i} \in \boldsymbol{Z}$ are replaced by their non-local counterpart $\tilde{Z}_{i} \in \tilde{Z}$, following

$$
\dot{\tilde{Z}}_{i}-\nabla_{0} \cdot\left(\mathrm{C}_{1 i} \cdot \nabla_{0} \dot{\tilde{Z}}_{i}\right)=\dot{Z}_{i} \text { on } \Omega_{0},
$$

where $\mathbf{C}_{1 i}$ is a semi-positive definite symmetric tensor associated to the non-local variable $\tilde{Z}_{i}$. In an isotropic case, it reduces to $l_{\mathrm{c}}^{2} \mathbf{I} ; l_{\mathrm{c}}$ being the associated characteristic diffusion length. Using $\tilde{Z}_{i}$ or its time derivative in Eq. (5) is analogous since the diffusion equation is linear. However, this latter formulation avoids numerical instabilities due to evolving boundary conditions during the crack propagation presented in Section 3. Furthermore, the local model is recovered when the characteristic length vanishes.

The differential equation (5) is completed by a natural boundary condition:

$$
\left(\mathrm{C}_{\mathrm{l} i} \cdot \nabla_{0} \dot{\tilde{Z}}_{i}\right) \cdot \boldsymbol{N}=0 \text { on } \Gamma_{0}
$$

\subsection{Hyperelastic-based elasto-plastic material model}

No assumption has been made yet on the material behaviour (4). First, as shown on Fig. 1, a classical multiplicative decomposition of the deformation gradient (1) for elasto-plastic materials is assumed

$$
\mathbf{F}=\mathbf{F}^{\mathrm{e}} \cdot \mathbf{F}^{\mathrm{p}},
$$

with $\mathbf{F}^{\mathrm{e}}$ the elastic part of $\mathbf{F}$ and $\mathbf{F}^{\mathrm{p}}$, the plastic one. The tensor $\mathbf{F}^{\mathrm{e}}$ can be separated into a rotational part $\mathbf{R}^{\mathrm{e}}$ and a symmetric part $\mathbf{U}^{\mathrm{e}}$ as $\mathbf{F}^{\mathrm{e}}=\mathbf{R}^{\mathrm{e}} \cdot \mathbf{U}^{\mathrm{e}}$. The corresponding Jacobian terms are defined by

$$
J=\operatorname{det} \mathbf{F}, J^{\mathrm{p}}=\operatorname{det} \mathbf{F}^{\mathrm{p}}, \text { and } J^{\mathrm{e}}=\operatorname{det} \mathbf{F}^{\mathrm{e}},
$$


and the corresponding right Cauchy strain tensors read

$$
\mathbf{C}=\mathbf{F}^{\mathrm{T}} \cdot \mathbf{F}, \mathbf{C}^{\mathrm{p}}=\mathbf{F}^{\mathrm{p} T} \cdot \mathbf{F}^{\mathrm{p}} \text {, and } \mathbf{C}^{\mathrm{e}}=\mathbf{F}^{\mathrm{eT}} \cdot \mathbf{F}^{\mathrm{e}} \text {. }
$$

The macro-mechanical response of the material, represented by the first Piola-Kirchhoff stress tensor $\mathbf{P}$, is determined by postulating the existence of a thermodynamic potential in terms of the conjugate strain tensor F. Assuming an isothermal rate-independent hyperelastic behaviour, the related free energy $\psi$ can be written under the form $\psi\left(\mathbf{C}^{\mathrm{e}}, \boldsymbol{Z}\right)^{1}$.

From the Clausius-Duhem inequality, the evolution of the free energy is bounded by

$$
\mathbf{P}: \dot{\mathbf{F}}-\dot{\psi}\left(\mathbf{C}^{\mathrm{e}}, \boldsymbol{Z}\right) \geq 0,
$$

where the equality is obtained in case of reversible elastic increments. This last equation is developed by using Eq. (7) and the form of $\psi$ as

$$
\mathbf{P}:\left(\dot{\mathbf{F}}^{\mathrm{e}} \cdot \mathbf{F}^{\mathrm{p}}\right)-\frac{\partial \psi}{\partial \mathbf{C}^{\mathrm{e}}}: \dot{\mathbf{C}}^{\mathrm{e}}+\mathbf{P}:\left(\mathbf{F}^{\mathrm{e}} \cdot \dot{\mathbf{F}}^{\mathrm{p}}\right)-\sum_{k} \frac{\partial \psi}{\partial Z_{k}} \dot{Z}_{k} \geq 0,
$$

or, after developing $\dot{\mathbf{C}}$, as

$$
\left(\mathbf{P}-2 \mathbf{F}^{\mathrm{e}} \cdot \frac{\partial \psi}{\partial \mathbf{C}^{\mathrm{e}}} \cdot \mathbf{F}^{\mathrm{p}-\mathrm{T}}\right):\left(\dot{\mathbf{F}}^{\mathrm{e}} \cdot \mathbf{F}^{\mathrm{p}}\right)+\mathbf{P}:\left(\mathbf{F}^{\mathrm{e}} \cdot \dot{\mathbf{F}}^{\mathrm{p}}\right)-\sum_{k} \frac{\partial \psi}{\partial Z_{k}} \dot{Z}_{k} \geq 0 .
$$

Assuming an arbitrary elastic reversible increment, the inequality becomes

$$
\left(\mathbf{P}-2 \mathbf{F}^{\mathrm{e}} \cdot \frac{\partial \psi}{\partial \mathbf{C}^{\mathrm{e}}} \cdot \mathbf{F}^{\mathrm{p}-\mathrm{T}}\right):\left(\dot{\mathbf{F}}^{\mathrm{e}} \cdot \mathbf{F}^{\mathrm{p}}\right)=0, \forall \dot{\mathbf{F}^{\mathrm{e}}}
$$

The PK1 stress is therefore defined by

$$
\mathbf{P}=2 \mathbf{F}^{\mathrm{e}} \cdot \frac{\partial \psi}{\partial \mathbf{C}^{\mathrm{e}}} \cdot \mathbf{F}^{\mathrm{p}-\mathrm{T}}
$$

and the Clausius-Duhem inequality (10) reduces to

$$
\mathbf{P}: \mathbf{F}^{\mathrm{e}} \cdot \dot{\mathbf{F}}^{\mathrm{p}}-\sum_{k} \frac{\partial \psi}{\partial Z_{k}} \dot{Z}_{k} \geq 0
$$

Using a logarithmic strain measure, i.e.,

$$
\mathbf{E}=\frac{1}{2} \ln \mathbf{C}, \mathbf{E}^{\mathrm{e}}=\frac{1}{2} \ln \mathbf{C}^{\mathrm{e}}, \text { and } \mathbf{E}^{\mathrm{p}}=\frac{1}{2} \ln \mathbf{C}^{\mathrm{p}},
$$

\footnotetext{
${ }^{1} \mathrm{~A}$ thermodynamic formulation accounting for the non-local variable has been derived by Nguyen et al. (Submitted) starting from the generalised micromorphic framework suggested by Forest (2009) but is omitted herein for conciseness
} 
the following isotropic quadratic potential is chosen

$$
\psi\left(\mathbf{C}^{\mathrm{e}}\right)=\frac{K}{2}\left[\operatorname{tr}\left(\mathbf{E}^{\mathrm{e}}\right)\right]^{2}+G\left(\mathbf{E}^{\mathrm{e}}\right)^{\operatorname{dev}}:\left(\mathbf{E}^{\mathrm{e}}\right)^{\operatorname{dev}},
$$

where $K=\frac{E}{3(1-2 \nu)}$ and $G=\frac{E}{2(1+\nu)}$ are the bulk and shear moduli of the material, based on the Young's modulus $E$ and the Poisson ratio $\nu$. Finally, applying the definition of $\psi(17)$ to Eq. (14) gives

$$
\mathbf{P}=K \operatorname{tr}\left(\mathbf{E}^{\mathrm{e}}\right) \mathbf{F}^{-\mathrm{T}}+2 G \mathbf{F}^{\mathrm{e}} \cdot \mathbf{C}^{\mathrm{e}-1} \cdot\left(\mathbf{E}^{\mathrm{e}}\right)^{\mathrm{dev}} \cdot \mathbf{F}^{\mathrm{p}-\mathrm{T}} .
$$

The Cauchy stress tensor $\boldsymbol{\sigma}$ is linked to the PK1 tensor by the classical relation

$$
\mathbf{P}=J \boldsymbol{\sigma} \cdot \mathbf{F}^{-\mathrm{T}}=\boldsymbol{\kappa} \cdot \mathbf{F}^{-\mathrm{T}},
$$

introducing $\boldsymbol{\kappa}$ as the Kirchhoff stress tensor. This latter is obtained from the rewriting of Eq. (18):

$$
\boldsymbol{\kappa}=K \operatorname{tr}\left(\mathbf{E}^{\mathrm{e}}\right) \mathbf{I}+2 G \mathbf{F}^{\mathrm{e}} \cdot \mathbf{C}^{\mathrm{e}-1} \cdot\left(\mathbf{E}^{\mathrm{e}}\right)^{\mathrm{dev}} \cdot \mathbf{F}^{\mathrm{eT}} .
$$

Because $\mathbf{U}^{\mathrm{e}}$ and $\mathbf{E}^{\mathrm{e}}$ commute, one has

$$
\boldsymbol{\kappa}=\mathbf{R}^{\mathrm{e}} \cdot(\underbrace{K \operatorname{tr}\left(\mathbf{E}^{\mathrm{e}}\right)}_{p^{\prime}} \mathbf{I}+\underbrace{2 G\left(\mathbf{E}^{\mathrm{e}}\right)^{\mathrm{dev}}}_{\boldsymbol{\tau}^{\mathrm{dev}}}) \cdot \mathbf{R}^{\mathrm{eT}}=\mathbf{R}^{\mathrm{e}} \cdot \boldsymbol{\tau} \cdot \mathbf{R}^{\mathrm{eT}},
$$

introducing $p^{\prime}$ as the pressure, $\boldsymbol{\tau}^{\text {dev }}$ as the deviatoric part of the corotational Kirchhoff stress tensor $\boldsymbol{\tau}$. The equivalent stress $\tau^{\mathrm{eq}}$ is defined as $\tau^{\mathrm{eq}}=\sqrt{\frac{3}{2} \boldsymbol{\tau}^{\mathrm{dev}}: \boldsymbol{\tau}^{\mathrm{dev}}}$.

\subsection{Porous elasto-plastic material model}

The elastic domain in stress space is limited by a yield function $\phi$. As already announced in the introduction, two modes of plastic deformation compete. Plastic flow can be diffuse in the matrix: it is then described by the Gurson model. Conversely, it can be localised between voids during the coalescence phase, it is described by the GTN model (i.e. GTN in the accelerated void growth phase) or by the Thomason model. By combining both modes of plastic flow, a full porous model is finally obtained.

\subsubsection{Generalities}

The description of the damage mechanism at the microscale is similar to Besson (2009): defects are assimilated as a periodic arrangement of spherical voids that stay spherical during the entire process and grow inside a J2-plastic matrix. A representative volume consists in a cuboid cell with a void at its centre as depicted on Fig. 2. Its geometrical state is fully described by 3 parameters: the porosity $f_{\mathrm{V}}$, the relative ligament ratio $\chi$ and the cell aspect ratio $\lambda$. The porosity $f_{\mathrm{V}}$ corresponds to the void volume fraction while the ligament ratio $\chi$ is the ratio of the void diameter to the distance between neighbouring voids. Although more complex and accurate microscopic descriptions exist, this one is chosen because of its simplicity. 


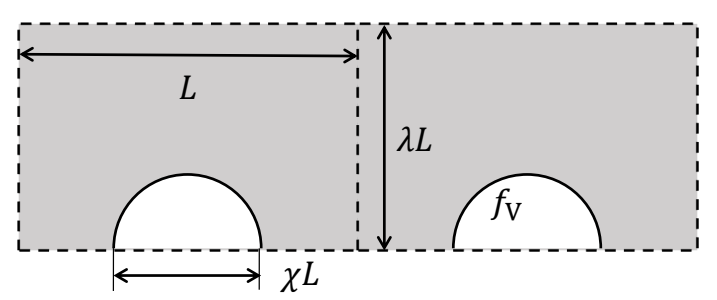

Figure 2: A representative volume cell and its geometrical description defined by the porosity $f_{\mathrm{V}}$, the cell aspect ratio $\lambda$ and the ligament ratio $\chi$.

Yield surface and plastic flow. Whatever the selected plasticity model, the yield condition $\phi \leqslant 0$ is here expressed in terms of the corotational Kirchhoff stress $\boldsymbol{\tau}$ instead of the classical Kirchhoff stress $\boldsymbol{\kappa}$. As the rate of the plastic flow is assumed to be irrotational, this change does not modify the plasticity model while it simplifies its implementation thanks to the straightforward relation between the stress tensor $\boldsymbol{\tau}$ and the logarithmic strain tensor $\mathbf{E}^{\mathrm{e}}$. The yield criterion depends also on the matrix yield stress. At this level, a classical J2plastic model is assumed with an isotropic hardening curve $\tau_{\mathrm{Y}}(\hat{p})$ in terms of the equivalent matrix plastic strain measure $\hat{p}$. The yield surface is also dependent on the microscopic state, represented by some effective variables $\breve{Z}$. The definition of the effective variables allows the transition from a non-local framework governed by $\tilde{Z}$ to a local one governed by $\boldsymbol{Z}$ without discontinuity. In particular, this set sums up the cumulative damage evolution during the homogeneous non-local damage process and, afterwards, during the local crack propagation.

The macroscopic yield criterion, based on stress invariants, is therefore written under the mathematical form

$$
\phi=\phi\left(\tau^{\mathrm{eq}}, p^{\prime}, \tau_{\mathrm{Y}}, \breve{Z}\right) \leqslant 0 .
$$

If the yield criterion (22) is satisfied, plastic flow occurs, following an associated flow rule. As the plastic flow is irrotational,

$$
\dot{\mathbf{F}^{\mathrm{p}}} \cdot \mathbf{F}^{\mathrm{p}-1}=\dot{\gamma} \mathbf{N}^{\mathrm{p}}=\dot{\gamma} \frac{\partial \phi}{\partial \boldsymbol{\tau}}
$$

where $\dot{\gamma} \geqslant 0$ is the macro-scale plastic multiplier associated with the plastic normal $\mathbf{N}^{\mathrm{p}}$. Since $\phi$ depends only on the first and second invariants of the stress tensor, the normal $\mathbf{N}^{\mathrm{p}}$ can be separated into a volumetric (or pressure) and a deviatoric part by stating $N^{\mathrm{d}}=\frac{\partial \phi}{\partial \tau^{\mathrm{eq}}}$ and $N^{\mathrm{q}}=\frac{\partial \phi}{\partial p^{\prime}}$ following

$$
\dot{\gamma} \mathbf{N}^{\mathrm{p}}=\dot{\gamma} \frac{\partial \phi}{\partial \tau^{\mathrm{eq}}} \frac{\partial \tau^{\mathrm{eq}}}{\partial \boldsymbol{\tau}}+\dot{\gamma} \frac{\partial \phi}{\partial p^{\prime}} \frac{\partial p^{\prime}}{\partial \boldsymbol{\tau}}=\dot{\gamma} N^{\mathrm{d}} \frac{3 \boldsymbol{\tau}^{\mathrm{dev}}}{2 \tau^{\mathrm{eq}}}+\dot{\gamma} N^{\mathrm{q}} \frac{1}{3} \mathbf{I}=\dot{\hat{d}} \frac{3 \boldsymbol{\tau}^{\mathrm{dev}}}{2 \tau^{\mathrm{eq}}}+\dot{\hat{q}} \frac{1}{3} \mathbf{I}
$$

where $\dot{\hat{d}}$ and $\dot{\hat{q}}$ respectively stand for $\dot{\gamma} N^{\mathrm{d}}$ and for $\dot{\gamma} N^{\mathrm{q}}$. 
Non-local variables. Concerning the introduction of the non-locality, the model focuses on diffusing one variable: $\tilde{Z}=\tilde{f}_{\mathrm{V}}$, as previously proposed by Reusch et al. (2003b,a). The related effective variable involved in the yield condition $(22), \breve{f}_{\mathrm{V}}$, is simply obtained through

$$
\dot{\vec{f}}=\dot{\tilde{V}}
$$

as long as the non-local model is used. Otherwise, its evolution is linked to the local porosity growth (see Section 3 for more details). The porosity is foreseen because this key parameter plays a key role in the entire damage and failure process. Among all other possibilities, Nguyen et al. (Submitted) adopted three non-local variables corresponding to the three plastic variables $(\hat{d}, \hat{q}, \hat{p})$ in order to capture void growth, coalescence by necking or shearing in a mesh-independent way. Another possible choice could be to diffuse the couple $\left(f_{\mathrm{V}}, \chi\right)$. Thereby, each mechanism will have its non-local key parameter associated with a corresponding non-local length. However, in this paper, mesh-dependency during the coalescence stage, linked to $\chi$, is already avoided by introducing a cohesive band model.

Internal variables evolution. The macroscopic deformation induces a microscopic structure modification and therefore, an evolution of the internal variables. The (local) porosity evolution $\dot{f}_{\mathrm{V}}$ is governed by different contributions all linked to the plastic flow

$$
\dot{f}_{\mathrm{V}}=\dot{f}_{\mathrm{V} \text { nucl }}+\dot{f}_{\mathrm{V} \text { growth }}+\dot{f}_{\mathrm{V}_{\text {shear }}} .
$$

Newly nucleated porosities $\Delta \dot{f}_{\mathrm{V} \text { nucl }}$ add to the already present porosity. This nucleation results from precipitate debonding or cracking during plastic flow. Among several existing models, a strain-controlled relation, presented by Chu and Needleman (1980), is used here: $\dot{f}_{\mathrm{V} \text { nucl }}=A_{\mathrm{n}}(\hat{p}) \dot{\hat{p}}$. Plastic flow also induces the growth of the voids $\dot{f}_{\mathrm{V} \text { growth }}$. As the macroscopic plastic flow is not purely deviatoric while the matrix volume is left unchanged by mass conservation, macroscopic plastic volume change induces a porosity growth rate $\dot{f}_{\mathrm{V} \text { growth }}=\left(1-f_{\mathrm{V}}\right) \operatorname{tr}\left(\dot{\gamma} \mathbf{N}^{\mathrm{p}}\right)$. Furthermore, the term $\dot{f}_{\mathrm{V}_{\text {shear }}}$ results from the extended Gurson model proposed by Nahshon and Hutchinson (2008). It corresponds to an apparent or effective void growth due to a shear-induced shape change with

$$
\dot{f}_{\mathrm{V}_{\text {shear }}}=B_{\mathrm{n}}(\zeta) \frac{\boldsymbol{\tau}^{\mathrm{dev}}: \dot{\gamma} \mathbf{N}^{\mathrm{p}}}{\tau^{\mathrm{eq}}} f_{\mathrm{V}}
$$

In this expression, $B_{\mathrm{n}}(\zeta)=k_{\omega}\left(1-\zeta^{2}\right)$ is a growth coefficient, controlled by the shear damage coefficient $k_{\omega}$, that vanishes under axisymmetric stress state and which is maximal under a combination of pure shear stress with an hydrostatic contribution. Nielsen and Tvergaard (2010) enhanced the coefficient by including a dependence on the triaxiality. Dunand and Mohr (2011) called $\zeta\left(\boldsymbol{\tau}^{\mathrm{dev}}\right)$ the normalised third invariant

$$
\zeta=\frac{27 \operatorname{det} \tau^{\operatorname{dev}}}{2 \tau^{\mathrm{eq} 3}} .
$$

The equivalent matrix plastic strain measure $\hat{p}$ is obtained by considering the energetic equivalence between both micro and macroscales:

$$
\left(1-f_{\mathrm{V}_{0}}\right) \tau_{\mathrm{Y}} \dot{\hat{p}}=\boldsymbol{\tau}: \dot{\gamma} \mathbf{N}^{\mathrm{p}}
$$


where $f_{\mathrm{V}_{0}}$, the initial porosity, is used since Eq. (29) is stated here with respect to the reference volume ${ }^{2}$.

The ligament size ratio $\chi$ is determined, following Besson (2009), from

$$
\chi=\left[\frac{3}{2} \breve{f}_{\mathrm{V}} \lambda\right]^{\frac{1}{3}}
$$

where $\breve{f}_{\mathrm{V}}$ is the effective porosity and the evolution of $\lambda$ is given by $\dot{\lambda}=\kappa \lambda \dot{\hat{p}}$. By integration, one thus has

$$
\chi=\left[\frac{3}{2} \breve{f}_{\mathrm{V}} \lambda_{0}\right]^{\frac{1}{3}} \exp \left(\frac{1}{3} \kappa \hat{p}\right)
$$

where $\lambda_{0}$ is the initial value of $\lambda$, the ratio of the void spacing arrangement, and where $\kappa$ is a fitting parameter (Besson, 2009).

The relationship (22) now needs to be particularised to the different considered porous plastic models while the features and equations common to each one have been presented.

\subsubsection{The Gurson micro-mechanical growth porous plastic model}

The void growth phase is described by the Gurson model for spherical voids (Tvergaard and Needleman, 1984). The corresponding yield surface $\phi_{\mathrm{G}}$ is given by

$$
\phi_{\mathrm{G}}\left(\tau^{\mathrm{eq}}, p^{\prime}, \tau_{\mathrm{Y}}, \breve{f}_{\mathrm{V}}\right)=\left(\frac{\tau^{\mathrm{eq}}}{\tau_{\mathrm{Y}}}\right)^{2}+2 \breve{f}_{\mathrm{V}} q_{1} \cosh \left(\frac{3 q_{2} p^{\prime}}{2 \tau_{\mathrm{Y}}}\right)-q_{3}^{2}\left(\breve{f}_{\mathrm{V}}\right)^{2}-1 \leqslant 0,
$$

where $q_{1}, q_{2}$ and $q_{3}$ are the Gurson parameters. Initially added by Tvergaard (1981), these parameters are determined to better fit cell experiments (Faleskog et al., 1998) and heuristically include several effects (e.g. strain hardening, void shape).

\subsubsection{Phenomenological coalescence porous plastic model}

After accumulation of plastic deformation and void growth, plastic flow localises between matrix voids, indicating the onset of void coalescence. It results in a significant change of the growth rate and overall deformation behaviour. This process can be described here by a phenomenological approach. In this case, the Gurson yield surface (32) is modified by a corrected porosity $f_{\mathrm{V}}^{\star}$ (Tvergaard and Needleman, 1984; Reusch et al., 2003b) once the coalescence criterion is met. The new coalescence yield surface is written

$$
\phi_{\mathrm{GTN}}=\phi_{\mathrm{G}}\left(\tau^{\mathrm{eq}}, p^{\prime}, \tau_{\mathrm{Y}}, f_{\mathrm{V}}^{\star}\right) \leqslant 0,
$$

where the corrected effective porosity $f_{\mathrm{V}}^{\star}$ is modified after the onset of coalescence by

$$
f_{\mathrm{V}}^{\star}\left(\breve{f}_{\mathrm{V}}\right)= \begin{cases}\breve{f}_{\mathrm{V}} & \text { if } \breve{f}_{\mathrm{V}} \leqslant f_{\mathrm{V}_{\mathrm{C}}} ; \\ f_{\mathrm{V}_{\mathrm{C}}}+\underbrace{f_{\mathrm{V}_{\mathrm{f}}}^{\star}-f_{\mathrm{V}_{\mathrm{C}}}}_{\delta_{\mathrm{GTN}}}\left(\breve{f}_{\mathrm{V}}-f_{\mathrm{V}_{\mathrm{C}}}\right) & \text { if } f_{\mathrm{V}_{\mathrm{C}}}<\breve{f}_{\mathrm{V}} \leqslant f_{\mathrm{V}_{\mathrm{f}}}\end{cases}
$$

\footnotetext{
${ }^{2}$ Rigorously speaking, this relation should also consider the nucleated porosity framed in the reference configuration, but this contribution is neglected herein.
} 
In Eq. (34), $f_{\mathrm{V}_{\mathrm{C}}}$ corresponds to the porosity value at the onset of coalescence. The value is fixed in advance and can either be considered as a material parameter (Tvergaard and Needleman, 1984) or correspond to the value predicted by a more physically-based coalescence criterion as the Thomason model (Zhang et al., 2000; Guzmán et al., 2018). After the onset of coalescence, the effective porosity growth rate $\dot{f}_{\mathrm{V}}^{\star}$ increases due to the acceleration rate $\delta_{\mathrm{GTN}}$. The porosity growth continues until the porosity $f_{\mathrm{V}_{\mathrm{f}}}$ at final failure, and $f_{\mathrm{V}_{\mathrm{f}}}^{\star}$ the corresponding corrected value, are reached. This coalescence model, besides the coalescence criterion, is defined either by $\delta_{\mathrm{GTN}}$ or by $f_{\mathrm{V}_{\mathrm{f}}}$. The last remaining parameter, $f_{\mathrm{V}_{\mathrm{f}}}^{\star}$, corresponds to a vanishing yield surface, i.e. $f_{\mathrm{V}}^{\star}\left(f_{\mathrm{V}_{\mathrm{f}}}\right)=f_{\mathrm{V}_{\mathrm{f}}}^{\star}=\frac{q_{1}-\sqrt{q_{1}^{2}-q_{3}^{2}}}{q_{3}^{2}}$.

\subsubsection{The Thomason micro-mechanical coalescence porous plastic model}

Another possibility to describe the coalescence behaviour after its onset is to use a yield surface, representative of the plastic flow process during coalescence. According to the Thomason model (Thomason, 1985a,b; Benzerga and Leblond, 2014), plastic flow occurs inside voids ligaments when the coalescence stress $C_{\mathrm{T}}^{\phi} \tau_{\mathrm{Y}}$, linked to the stress concentration factor $C_{\mathrm{T}}^{\phi}$ (due to void configuration) and the matrix flow stress $\tau_{\mathrm{Y}}$ inside the localisation zone, is reached (Besson, 2009); i.e., assuming a traction along direction $z z$, the yield surface reads

$$
\tau_{z z} \leqslant C_{\mathrm{T}}^{\phi}(\chi) \tau_{\mathrm{Y}}
$$

Equation (35) can be rewritten for any arbitrary orientation (Pardoen and Hutchinson, 2000) in terms of the corotational Kirchhoff stress as

$$
\phi_{\mathrm{T}^{+}}=\frac{2}{3} \tau^{\mathrm{eq}}+p^{\prime}-C_{\mathrm{T}}^{\phi}(\chi) \tau_{\mathrm{Y}} \leqslant 0 .
$$

Conversely, plastic flow can also occur in compression, resulting in a supplementary yield surface

$$
\phi_{\mathrm{T}^{-}}=\frac{2}{3} \tau^{\mathrm{eq}}-p^{\prime}-C_{\mathrm{T}}^{\phi}(\chi) \tau_{\mathrm{Y}} \leqslant 0 .
$$

In order to ensure a smooth yield surface and remove vertices, Besson (2009) used an interpolation between both $\phi_{\mathrm{T}^{+}}$and $\phi_{\mathrm{T}^{-}}$, leading finally to

$$
\phi_{\mathrm{T}}\left(\tau^{\mathrm{eq}}, p^{\prime}, \tau_{\mathrm{Y}}, \chi\right)=[\underbrace{\left(\frac{2}{3} \tau^{\mathrm{eq}}+p^{\prime}\right)^{n}}_{\tau_{+}}+\underbrace{\left(\frac{2}{3} \tau^{\mathrm{eq}}-p^{\prime}\right)^{n}}_{\tau_{-}}]^{1 / n}-C_{\mathrm{T}}^{\phi}(\chi) \tau_{\mathrm{Y}} \leqslant 0,
$$

with $n$ a sufficiently high even integer interpolation exponent to stay close to the original surfaces.

The concentration factor $C_{\mathrm{T}}^{\phi}$ is given in terms of the ligament size ratio $\chi$,

$$
C_{\mathrm{T}}^{\phi}(\chi)=\left(1-\chi^{2}\right)\left[\alpha(1 / \chi-1)^{2}+\beta \sqrt{1 / \chi}\right],
$$

where the parameters $\alpha$ and $\beta$ depend on the strain-hardening exponent $h_{\exp }$ and are respectively equal to $0.1+0.217 h_{\exp }+4.83 h_{\exp }^{2}$ and 1.24 for $0 \leqslant h_{\exp } \leqslant 0.3$ as calibrated by Pardoen and Hutchinson (2000). 


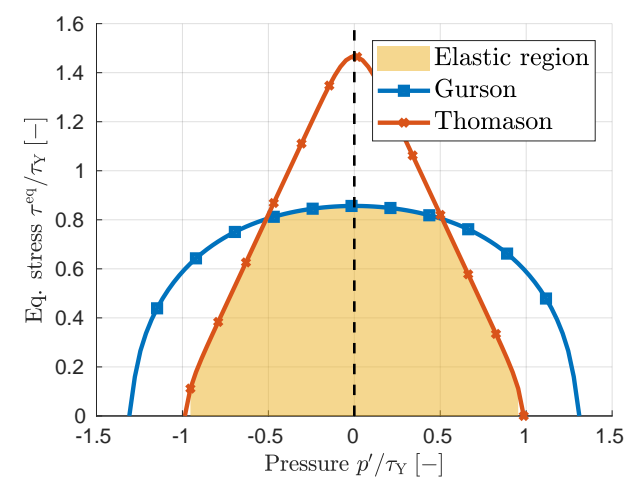

Figure 3: The two-yield surface model combining the Gurson yield surface $\phi_{\mathrm{G}}$ and the Thomason yield model $\phi_{\mathrm{T}}$ in the $\left(p^{\prime}, \tau^{\mathrm{eq}}\right)$ space.

\subsubsection{A complete porous elastoplastic model: the Gurson-Thomason model}

The full porous model is obtained when both diffuse and localised mechanisms are taken into account. First, the "growth" mode and its corresponding yield surface (32) is active. Afterwards, the coalescence mode (with the yield surface (38)) becomes dominant. During the transition, both modes can be active by considering a two-surface model: at this point, the effective elastic region results in their intersection as shown on Fig. 3. The yield condition can be summarised by

$$
\phi_{\mathrm{GT}}=\max \left(\phi_{\mathrm{G}}, \phi_{\mathrm{T}}\right) \leqslant 0 .
$$

A particular treatment has to be applied to remove surface vertices and to ensure a unique and continuous $\mathbf{N}^{\mathrm{P}}$ between iterations for convergence purpose. Therefore, the management of the intersection the yield surfaces can be done by interpolation (as similarly done for $\phi_{\mathrm{T}}$ ) as suggested by Nguyen et al. (Submitted), or by using a cone of normals. A simpler choice is made here: the current active yield surface is selected at the end of the previous time step. This choice is justified because the coalescence surface shrinks faster than the void growth one. Once the coalescence is activated, oscillations between both strain modes are mainly limited to the onset stage.

\subsection{Numerical integration of the constitutive law}

The constitutive material law is integrated numerically following a backward Euler scheme. Before being adapted to the local model in Section 2.4.4, the methodology is first presented in the context of the non-local model, i.e. $\breve{Z}=\tilde{Z}$ or in this case $\breve{f}_{\mathrm{V}}=\tilde{f}_{\mathrm{V}}$. The objective is therefore to compute the stress state and the local internal variables at time $t^{n+1}$ (i.e. $\left.\left(\mathbf{P}^{n+1}, f_{\mathrm{V}}^{n+1}\right)\right)$ from the known state at the previous discrete time step $t^{n}$ and from the actual deformation gradient $\mathbf{F}^{n+1}$ and non-local variable $\tilde{f}_{\mathrm{V}}^{n+1}$.

The procedure is summarised in Fig. 4. Using Eqs. (18) to (21), the problem is solved in terms of the $\boldsymbol{\tau}$ stress and the plastic deformation $\mathbf{F}^{\mathrm{p}}$. The time integration on the interval $\left[t^{n}, t^{n+1}\right]$ is done using a predictor-corrector scheme similar to the one described by Cuitino 
and Ortiz (1992). At first, the strain increment is assumed to be purely elastic (Section 2.4.1). If this predictor stress is outside the yield surface, a plastic flow occurs: a plastic correction is therefore applied following the implicit radial return-like algorithm presented in Section 2.4.2. Section 2.4.3 provides in details the derivation of the material tangent operators. If a solution cannot be found, a classical linear sub-stepping scheme is used to solve the problem on smaller intervals $\left[t^{n^{\prime}}, t^{n^{\prime}+1}\right] \subset\left[t^{n}, t^{n+1}\right]$. In the following lines, the superscript $n+1$ used to refer to the variables evaluated at this time is omitted for readability.

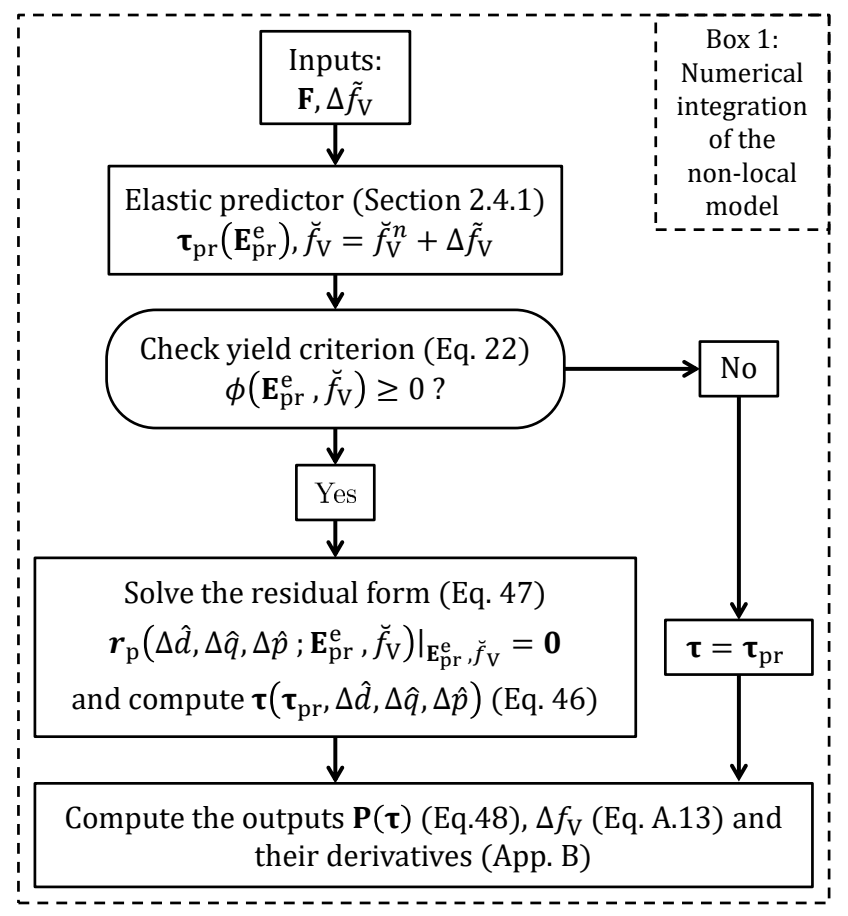

Figure 4: Numerical integration procedure of the non-local constitutive porous-plastic model (material model level).

\subsubsection{Elastic predictor}

During the predictor step, the plastic deformation gradient is kept unchanged $\mathbf{F}_{\mathrm{pr}}^{\mathrm{p}}{ }^{n+1}=$ $\mathbf{F}^{\mathrm{p}^{n}}$ as well as the other variables linked to plasticity. The stress state is computed following Eqs. (7) to (21), leading to the elastic predictor of the deformation gradient

$$
\mathbf{F}_{\mathrm{pr}}^{\mathrm{e}}=\mathbf{F} \cdot \mathbf{F}_{\mathrm{pr}}^{\mathrm{p}-1}
$$

and the strain predictor tensors

$$
\mathbf{C}_{\mathrm{pr}}^{\mathrm{e}}=\mathbf{F}_{\mathrm{pr}}^{\mathrm{e}}{ }^{\mathrm{T}} \cdot \mathbf{F}_{\mathrm{pr}}^{\mathrm{e}} \text { and } \mathbf{E}_{\mathrm{pr}}^{\mathrm{e}}=\frac{1}{2} \ln \left(\mathbf{C}_{\mathrm{pr}}^{\mathrm{e}}\right) \text {. }
$$

The predictor stress components then read

$$
\tau_{\mathrm{pr}}^{\mathrm{dev}}=2 G\left(\mathbf{E}_{\mathrm{pr}}^{\mathrm{e}}\right)^{\text {dev }} \underset{16}{\text { and }} p_{\mathrm{pr}}^{\prime}=K \operatorname{tr}\left(\mathbf{E}_{\mathrm{pr}}^{\mathrm{e}}\right) .
$$




\subsubsection{Plastic corrector}

The yield condition (22) is assessed using the predictor state (43). If the criterion is satisfied, the elastic predictor state corresponds to the final one: $\mathbf{F}^{\mathrm{p}}=\mathbf{F}_{\mathrm{pr}}^{\mathrm{p}}$ and $\boldsymbol{\tau}=\boldsymbol{\tau}_{\mathrm{pr}}$. Otherwise, a plastic correction is needed. Following the detailed formulation presented in Appendix A, the current plastic deformation gradient reads

$$
\mathbf{F}^{\mathrm{p}}=\exp \left(\Delta \gamma \mathbf{N}^{\mathrm{p}}\right) \cdot \mathbf{F}_{\mathrm{pr}}^{\mathrm{p}} .
$$

Using the flow decomposition (24), the plastic increment is given by

$$
\Delta \gamma \mathbf{N}^{\mathrm{p}}=\Delta \hat{d} \frac{3 \tau^{\mathrm{dev}}}{2 \tau^{\mathrm{eq}}}+\Delta \hat{q} \frac{1}{3} \mathbf{I}
$$

where $\Delta \hat{d}$ is the increment of the deviatoric macroscopic plastic flow and $\Delta \hat{q}$ of the macroscopic volumetric part. The corrected stress state after plastic flow (see Appendix A.1 for more details) then reads

$$
\tau^{\mathrm{eq}}=\tau_{\mathrm{pr}}^{\mathrm{eq}}-3 G \Delta \hat{d}, \boldsymbol{\tau}^{\mathrm{dev}}=\frac{\tau_{\mathrm{pr}}^{\mathrm{eq}}-3 G \Delta \hat{d}}{\tau_{\mathrm{pr}}^{\mathrm{eq}}}\left(\boldsymbol{\tau}_{\mathrm{pr}}\right)^{\mathrm{dev}}, \text { and } p^{\prime}=p_{\mathrm{pr}}^{\prime}-K \Delta \hat{q} .
$$

Using Eqs. (45)-(46), the corrector step can be summarised as finding, for a given couple $\left(\mathbf{E}_{\mathrm{pr}}^{\mathrm{e}}, \tilde{f}_{\mathrm{V}}\right)$, the macroscopic plastic increments $(\Delta \hat{d}, \Delta \hat{q})$ and the microscopic one $\Delta \hat{p}$ that simultaneously satisfy the yield condition (22), the normality rule (23), the equivalence of the plastic power between scales (29), and the evolution laws of the internal variables, or

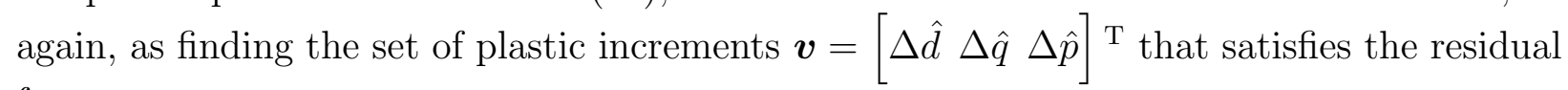
form

$$
\left.\boldsymbol{r}_{\mathrm{p}}\left(\Delta \hat{d}, \Delta \hat{q}, \Delta \hat{p} ; \mathbf{E}_{\mathrm{pr}}^{\mathrm{e}}, \tilde{f}_{\mathrm{V}}\right)\right|_{\mathbf{E}_{\mathrm{pr}}^{\mathrm{e}}, \tilde{f}_{\mathrm{V}}}=\mathbf{0}
$$

which is solved using an iterative Newton-Raphson procedure (see Appendix A.2 for more details). Once the stress state $\boldsymbol{\tau}$ and the plastic strain $\mathbf{F}^{\mathrm{p}}$ are obtained, $\mathbf{P}$ is finally recovered from Eq. (18):

$$
\mathbf{P}=\mathbf{F}^{\mathrm{e}} \cdot\left(\boldsymbol{\tau}: \mathcal{L}^{\mathrm{e}}\right) \cdot \mathbf{F}^{\mathrm{p}-\mathrm{T}},
$$

with $\mathcal{L}^{\mathrm{e}}=\frac{\partial \ln \mathbf{C}^{\mathrm{e}}}{\partial \mathbf{C}^{\mathrm{e}}}$ dependent on the approximation involved when computing the function $\ln \mathbf{C}^{\mathrm{e}}$.

\subsubsection{Material linearisation}

The constitutive model needs to provide the material tangent operators. Practically, these are obtained by linearising the output of the material law $\left(\mathbf{P}, f_{\mathrm{V}}\right)$, respectively given by Eqs. (48) and (26) (see the integral form in Appendix A.2) in terms of the input $\left(\mathbf{F}, \tilde{f}_{\mathrm{V}}\right)$. Using the chain rule and tensorial calculus, the problem can be re-expressed in terms of the derivatives of the plastic increments $\boldsymbol{v}$. In this purpose, the residual vector (47) is linearised in terms of the predictor strain $\mathbf{E}_{\mathrm{pr}}^{\mathrm{e}}$ and of the porosity $\tilde{f}_{\mathrm{V}}$ :

$$
\dot{\boldsymbol{r}_{\mathrm{p}}}\left(\boldsymbol{v} ; \mathbf{E}_{\mathrm{pr}}^{\mathrm{e}}, \tilde{f}_{\mathrm{V}}\right)=\mathbf{0}=\frac{\partial \boldsymbol{r}_{\mathrm{p}}}{\partial \boldsymbol{v}} \dot{\boldsymbol{v}}+\frac{\partial \boldsymbol{r}_{\mathrm{p}}}{\partial \mathbf{E}_{\mathrm{pr}}^{\mathrm{e}}}: \dot{\mathbf{E}}_{\mathrm{pr}}^{\mathrm{e}}+\frac{\partial \boldsymbol{r}_{\mathrm{p}}}{\partial \tilde{f}_{\mathrm{V}}} \dot{\tilde{f}}_{\mathrm{V}}=\mathbf{0}
$$


from which the derivatives can be easily extracted following the definition of the Jacobian matrix $\mathbf{J}_{\boldsymbol{v}}=\frac{\partial \boldsymbol{r}_{\mathrm{p}}}{\partial \boldsymbol{v}}$ explicited in Appendix A.2, with

$$
\frac{\partial v_{i}}{\partial \mathbf{E}_{\mathrm{pr}}^{\mathrm{e}}}=-J_{v_{i j}}^{-1} \frac{\partial r_{\mathrm{p}_{j}}}{\partial \mathbf{E}_{\mathrm{pr}}^{\mathrm{e}}} \text { and } \frac{\partial v_{i}}{\partial \tilde{f}_{\mathrm{V}}}=-J_{v i j}^{-1} \cdot \frac{\partial r_{\mathrm{p}_{j}}}{\partial \tilde{f}_{\mathrm{V}}} .
$$

The derivatives in terms of the predictor strain can then be replaced in terms of the deformation gradient, see Appendix B for more details.

\subsubsection{Local form of the porous model}

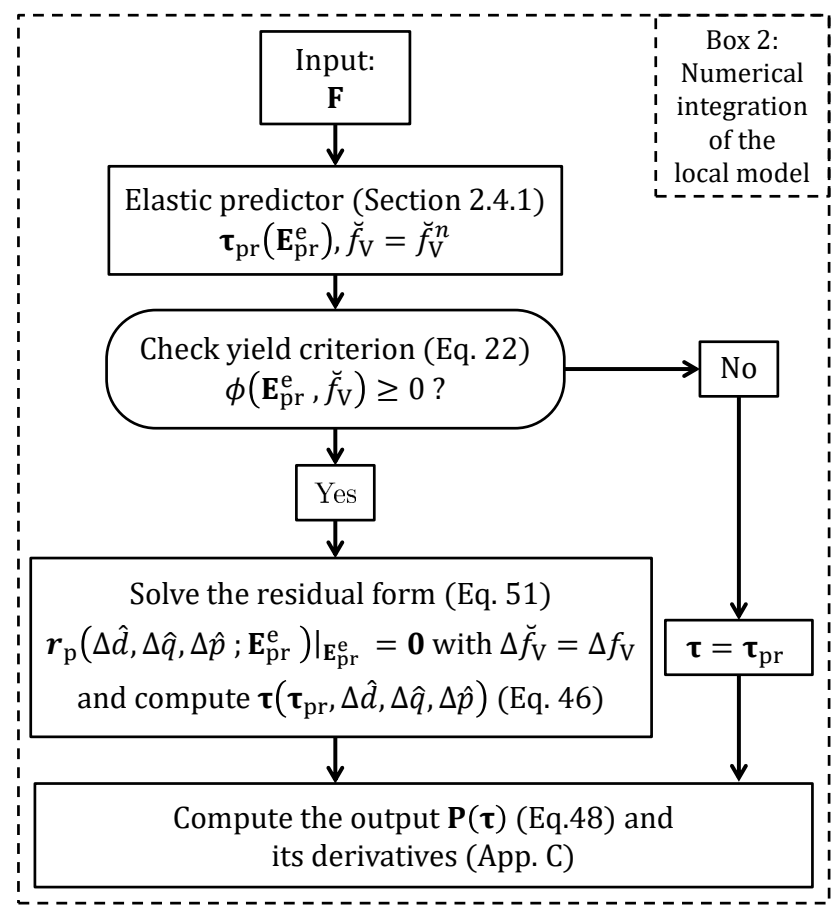

Figure 5: Numerical integration procedure of the local version of the constitutive porous-plastic model (material model level).

The results of Sections 2.4.2 and 2.4.3 are now adapted under a local form, i.e. $\breve{Z}=\boldsymbol{Z}$ or in this case $\breve{f}_{\mathrm{V}}=f_{\mathrm{V}}$. The resulting procedure is summarised in Fig. 5. Henceforth, the effective variables increments are no longer dependent on the variations of the non-local values, and the stress state $\mathbf{P}^{n+1}$ is computed from the deformation gradient only. The residual form, from which the set of plastic increments $\boldsymbol{v}$ is obtained, is slightly modified

$$
\left.\boldsymbol{r}_{\mathrm{p}}\left(\Delta \hat{d}, \Delta \hat{q}, \Delta \hat{p} ; \mathbf{E}_{\mathrm{pr}}^{\mathrm{e}}\right)\right|_{\mathbf{E}_{\mathrm{pr}}^{\mathrm{e}}}=\mathbf{0}
$$

as well as the corresponding Jacobian matrix, both being detailed in Appendix C. The material tangent operator, $\frac{\partial \mathbf{P}}{\partial \mathbf{F}}$, is consequently reformulated following the same procedure as in Section 2.4.3, see Appendix C.2 for more details. 


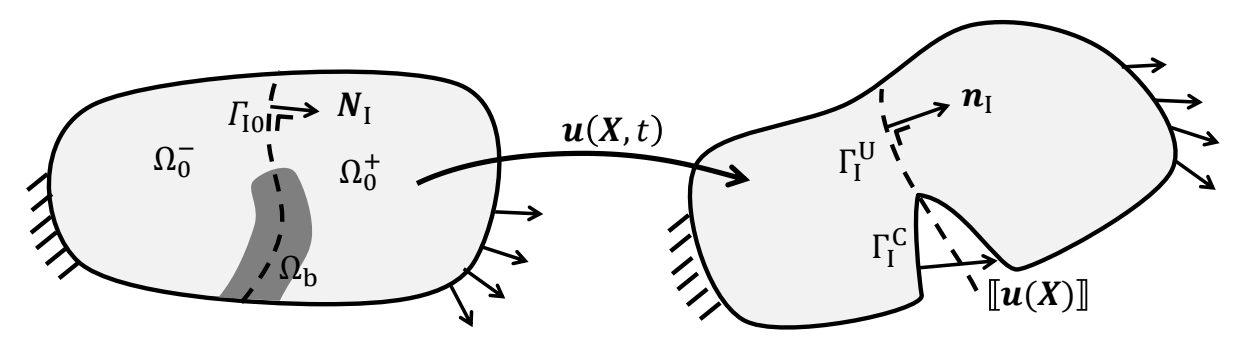

Figure 6: The solid is embedding a discontinuity surface $\Gamma_{\mathrm{I} 0}$, encompassed in the fictitious cohesive band $\Omega_{\mathrm{b} 0}$. The discontinuity separates the volume into two parts $\Omega_{0}^{+}$and $\Omega_{0}^{-}$as depicted in the reference configuration (left) and in the current configuration (right).

\section{The diffuse damage to crack transition}

Crack surfaces are introduced in the mechanical problem through a cohesive band model. For this purpose, the classical cohesive model is taken as a starting point and extended into a cohesive band formulation in the spirit of the work of Leclerc et al. (2018) achieved in the context of elastic damage. The crack insertion criterion and the calibration of the cohesive band thickness are explained next.

\subsection{Cohesive band model}

In the present formulation, multiple crack surfaces can be introduced in the material body $\Omega$. Without any loss of generality, let us assume that one discontinuity surface $\Gamma_{\text {I0 }}$ follows a given trajectory inside the reference configuration. At a given time $t$, the discontinuity path is divided into an already cracked part $\Gamma_{\mathrm{I} 0}^{\mathrm{C}}$ and an uncracked one $\Gamma_{\mathrm{I} 0}^{\mathrm{U}}$, satisfying $\Gamma_{\mathrm{I} 0}^{\mathrm{U}} \cap \Gamma_{\mathrm{I} 0}^{\mathrm{C}}=0$ and $\Gamma_{\mathrm{I} 0}^{\mathrm{U}} \cup \Gamma_{\mathrm{I} 0}^{\mathrm{C}}=\Gamma_{\mathrm{I} 0}$. The volume $\Omega_{0}$ is now separated into two parts $\Omega_{0}^{+}$and $\Omega_{0}^{-}$, as illustrated on Fig. 6 . The unit normal $\boldsymbol{N}_{\mathrm{I}}$ to $\Gamma_{\text {I } 0}$ is oriented towards $\Omega_{0}^{+}$. The counterpart of $\boldsymbol{N}_{\mathrm{I}}$ in the current configuration is $\boldsymbol{n}_{\mathrm{I}}$. In $3 \mathrm{D}$, two unit tangential vectors $\boldsymbol{T}_{\mathrm{I}}$ and $\boldsymbol{S}_{\mathrm{I}}$ form an orthonormal basis with $\boldsymbol{N}_{\mathrm{I}}$.

In the following equations, two operators are introduced at the interface to link variables $\bullet+$ and $\bullet-$ from both sides of the crack $\Gamma_{\mathrm{I} 0}$ :

$$
\begin{aligned}
& \text { the jump operator: } \quad \llbracket \bullet=\left[\bullet^{+}-\bullet^{-}\right], \quad \text { and } \\
& \text { the mean operator: } \quad\langle\bullet\rangle=\frac{1}{2}\left[\bullet^{+}+\bullet^{-}\right] .
\end{aligned}
$$

The displacement field is not continuous across the cracked surfaces $\Gamma_{\mathrm{I} 0}^{\mathrm{C}}$ anymore: two initially neighbouring points at the same location $\boldsymbol{X}$ on $\Gamma_{\text {I0 }}$ are now separated by $\llbracket \boldsymbol{u}(\boldsymbol{X}) \rrbracket$. To satisfy internal equilibrium, the surface traction forces in the current configuration per unit of surface in the reference configuration, $\boldsymbol{t}_{\mathrm{b} 0}$, have to be continuous across the surface:

$$
\llbracket \boldsymbol{t}_{\mathrm{b} 0} \rrbracket=\mathbf{0} \text { on } \Gamma_{\mathrm{I} 0}^{\mathrm{C}},
$$

while this internal boundary surface is treated as a free boundary surface from a non-local point of view (similarly to Eq. (6)):

$$
\left(\mathrm{C}_{l} \cdot \nabla_{0} \dot{\tilde{Z}}_{i}\right) \cdot \boldsymbol{N}_{\mathrm{I}}=0 \text { on } \Gamma_{\mathrm{I} 0}^{\mathrm{C}} .
$$


The evolution of the traction force $\boldsymbol{t}_{\mathrm{b} 0}$ is defined by the cohesive model. Pioneered by Dugdale (1960) and Barenblatt (1962), this representation of the material response locally dissipates energy by crack surface creation. As the crack gradually opens and the jump $\llbracket \boldsymbol{u} \rrbracket$ increases, the bounding traction forces $\boldsymbol{t}_{\mathrm{b} 0}$ between crack lips irreversibly decrease and dissipate an amount of energy per unit of created surface in the reference configuration, $G_{\mathrm{c}}$ (so-called, critical energy release rate)

$$
G_{\mathrm{c}}=\int_{0}^{\infty} \boldsymbol{t}_{\mathrm{b} 0}(\llbracket \boldsymbol{u} \rrbracket) \cdot d \llbracket \boldsymbol{u} \rrbracket .
$$

The cohesive law simply defines the relationship $\boldsymbol{t}_{\mathrm{b} 0}(\llbracket \boldsymbol{u} \rrbracket)$ between the traction force and the crack opening.

As already discussed by Leclerc et al. (2018) in the context of elastic damage, the original cohesive zone approach is intrinsically surfacic. By contrast, the damage model presented earlier is volumic and depends on the 3D stress state. Moreover, near failure, all the damage growth tends to concentrate within a band around the future crack surface. This band, where coalescence typically occurs, is very thin, about a few micro-voids thick (Pineau et al., 2016), but still of finite thickness. These physical facts can be included by replacing the classical CZM by the cohesive band model (Remmers et al., 2013; Huespe et al., 2012). While respecting a traction-separation model formalism, this latter mimics the behaviour of a uniform thin band around the crack surface. A stress tensor is computed by using the underlying damage model with an enhanced strain tensor at the interface.

As the displacement field $\boldsymbol{u}(\boldsymbol{X})$ is discontinuous at the interface, the deformation gradient cannot be directly defined at the discontinuous surface $\Gamma_{\mathrm{I} 0}^{\mathrm{C}}$. The field is thus divided into a continuous component $\boldsymbol{u}_{\mathrm{c}}(\boldsymbol{X})$ and a discontinuous one $\llbracket \boldsymbol{u}(\boldsymbol{X}) \rrbracket$ in the neighbourhood of $\Gamma_{\mathrm{I} 0}^{\mathrm{C}}$ :

$$
\boldsymbol{u}(\boldsymbol{X})=\boldsymbol{u}_{\mathrm{c}}(\boldsymbol{X})+\mathcal{H}_{\mathrm{D}}(\boldsymbol{X}) \llbracket \boldsymbol{u}(\boldsymbol{X}) \rrbracket,
$$

introducing $\mathcal{H}_{\mathrm{D}}$, the Heavyside's function related to the crack surface

$$
\mathcal{H}_{\mathrm{D}}(\boldsymbol{X})= \begin{cases}0 & \text { if } \boldsymbol{X} \in \Omega_{0}^{-} \\ 1 & \text { if } \boldsymbol{X} \in \Omega_{0}^{+}\end{cases}
$$

In the context of a finite thickness band, the discontinuous contribution is assumed to be smeared and smoothed through the thickness $h_{\mathrm{b}}$ of the cohesive band $\Omega_{\mathrm{b} 0}$ encompassing $\Gamma_{\mathrm{I} 0}^{\mathrm{C}}$. If we assume $\Gamma_{\mathrm{I} 0}^{\mathrm{C}}$ to be locally planar $^{3}$, the displacement field can be rewritten as

$$
\boldsymbol{u}(\boldsymbol{X})=\boldsymbol{u}_{\mathrm{c}}(\boldsymbol{X})+\mathcal{H}_{\mathrm{B}}(\boldsymbol{X}) \llbracket \boldsymbol{u}(\boldsymbol{X}) \rrbracket,
$$

where the continuous function $\mathcal{H}_{\mathrm{B}}(\boldsymbol{X})$ has replaced the discontinuous one $\mathcal{H}_{\mathrm{D}}$. It corresponds to a piece-wise linear-step:

$$
\mathcal{H}_{\mathrm{B}}(\boldsymbol{X})= \begin{cases}0 & \text { if } \boldsymbol{X} \in \Omega_{0}^{-} \backslash \Omega_{\mathrm{b} 0} \\ \frac{\left(\boldsymbol{X}-\boldsymbol{X}_{\mathrm{I}}\right) \cdot \boldsymbol{N}_{\mathrm{I}}}{h_{\mathrm{b}}}+\frac{1}{2} & \text { if } \boldsymbol{X} \in \Omega_{\mathrm{b} 0} \\ 1 & \text { if } \boldsymbol{X} \in \Omega_{0}^{+} \backslash \Omega_{\mathrm{b} 0}\end{cases}
$$

\footnotetext{
${ }^{3}$ i.e. on a distance $\geqslant h_{\mathrm{b}}$
} 
where $\boldsymbol{X}_{\mathrm{I}}$ is a point on the mid plane of the band, on the crack surface. By considering this smoothing of the jump along the fictitious thickness $h_{\mathrm{b}}$, a strain band deformation gradient $\mathbf{F}_{\mathrm{b}}$ is recovered by differentiation of Eq. (58):

$$
\mathbf{F}_{\mathrm{b}}=\mathbf{I}+\frac{\partial \boldsymbol{u}_{\mathrm{c}}(\boldsymbol{X})}{\partial \boldsymbol{X}}+\frac{1}{h_{\mathrm{b}}} \llbracket \boldsymbol{u}(\boldsymbol{X}) \rrbracket \otimes \boldsymbol{N}_{\mathrm{I}}+\left(\frac{\left(\boldsymbol{X}-\boldsymbol{X}_{\mathrm{I}}\right) \cdot \boldsymbol{N}_{\mathrm{I}}}{h_{\mathrm{b}}}+\frac{1}{2}\right) \frac{\partial \llbracket \boldsymbol{u}(\boldsymbol{X}) \rrbracket}{\partial \boldsymbol{X}} .
$$

The jump contribution of the deformation gradient is assumed to be uniform along the band thickness $h_{\mathrm{b}}$, considered very small compared to its transverse dimensions. Therefore, the continuous displacement part in Eq. (60) can be approximated on both sides $\boldsymbol{X}_{\mathrm{I}}^{ \pm}$of the crack surface, according with the chosen DG-discretisation (see Section 4). The same approximation is done for the last term. The band deformation gradient is thus equal to

$$
\mathbf{F}_{\mathrm{b}}^{ \pm}=\mathbf{F}^{ \pm}+\frac{1}{h_{\mathrm{b}}} \llbracket \boldsymbol{u} \rrbracket \otimes \boldsymbol{N}_{\mathrm{I}}+\frac{1}{2} \frac{\partial \llbracket \boldsymbol{u} \rrbracket}{\partial \boldsymbol{X}},
$$

with $\mathbf{F}^{ \pm}=\mathbf{F}\left(\boldsymbol{X}_{\mathrm{I}}{ }^{ \pm}\right)$evaluated on both sides of the band. In this last equation, the displacement discontinuity $\llbracket \boldsymbol{u}(\boldsymbol{X}) \rrbracket$ is assumed constant across the thickness, i.e

$$
\frac{\partial \llbracket \boldsymbol{u}(\boldsymbol{X}) \rrbracket}{\partial \boldsymbol{X}} \cdot \boldsymbol{N}_{\mathbf{I}}=\mathbf{0} .
$$

The stress tensor $\mathbf{P}_{\mathrm{b}}^{ \pm}$on both sides of the interface is computed using the constitutive bulk law in its local form

$$
\mathbf{P}_{\mathrm{b}}^{ \pm}(t)=\mathbf{P}_{\mathrm{b}}^{ \pm}\left(\mathbf{F}_{\mathrm{b}}^{ \pm}(t) ; \breve{Z}^{ \pm}(t), \boldsymbol{Z}^{ \pm}\left(t^{\prime}\right), t^{\prime} \in[0, t]\right),
$$

where $\mathbf{F}_{\mathrm{b}}$ replaces the classical deformation gradient, and where the effective internal variables depend henceforth only on the local evolution after crack insertion, i.e. $\dot{\breve{Z}}=\dot{Z}$, see Section 2.4.4. This relation guarantees a continuous traction force on the interface during the transition. The cohesive traction forces are finally obtained by:

$$
\boldsymbol{t}_{\mathrm{b} 0}=\mathbf{P}_{\mathrm{b}} \cdot \mathbf{N}_{\mathrm{I}}
$$

Thereby, the cohesive forces are naturally defined in terms of the underlying material behaviour and the in-plane stretch components. Stress triaxiality effects are therefore included inside the cohesive law within a formalism similar to a classical cohesive zone model. The whole procedure is summarised in Fig. 7. In the context of a hybrid DG/CZM framework, only a few changes are required to integrate this model, as the necessary Gauss points are already present at the interface and as a stress tensor is already computed at the interface before crack propagation, see Section 4.

\subsection{Crack insertion criterion}

A crack insertion criterion is now needed to decide how and when cracks should propagate on the uncracked surface $\Gamma_{\mathrm{I} 0}^{\mathrm{U}}$. A suitable condition has (i) to ensure a softening behaviour after insertion and an elastic unloading in the neighbouring elements, (ii) to predict the propagation direction. In this framework, two possibilities are selected. 


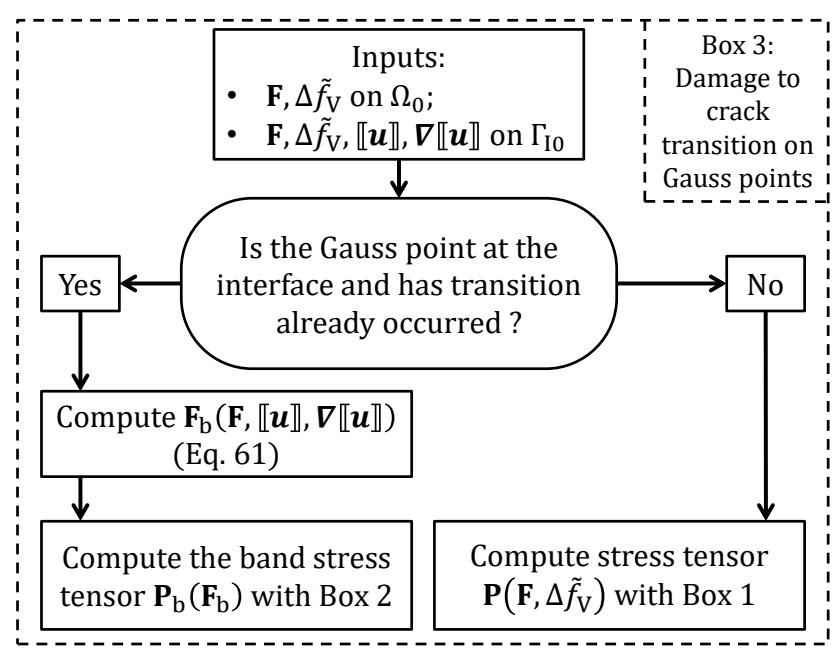

Figure 7: The non-local diffuse damage to crack transition algorithm, including the cohesive band model, in the context of the porous-plastic model.

\subsubsection{Loss of ellipticity for local models}

In the context of a local model, a requirement to keep solution uniqueness is to introduce the CBM at the loss of strong ellipticity as detected using the acoustic tensor. This criterion will allow the comparison of the results with the simulation performed by Huespe et al. (2012) in the context of local models. Following Huespe et al. (2012), the strong ellipticity is lost at a given point once

$$
\operatorname{det}\left[\boldsymbol{N} \cdot 2 \frac{\partial \mathbf{P}}{\partial \mathbf{F}} \cdot \boldsymbol{N}\right] \leqslant 0
$$

for any direction vector $\boldsymbol{N}$. The crack insertion is constrained along an interface perpendicular to the interface normal $\boldsymbol{N}_{\mathrm{I}}$, i.e.

$$
\operatorname{det}\left[\boldsymbol{N}_{\mathrm{I}} \cdot 2 \frac{\partial \mathbf{P}}{\partial \mathbf{F}} \cdot \boldsymbol{N}_{\mathrm{I}}\right] \leqslant 0
$$

or, similarly

$$
-\min \left(\operatorname{eig}\left[\boldsymbol{N}_{\mathrm{I}} \cdot \frac{\partial \mathbf{P}}{\partial \mathbf{F}} \cdot \boldsymbol{N}_{\mathrm{I}}\right]\right) \geqslant \epsilon \geqslant 0
$$

where the operator ".2" stands for $\left[\mathbf{A} \cdot{ }^{2} \mathcal{B}\right]_{i j k l}=A_{j m} \mathcal{B}_{i m k l}$, the operator "eig" computes the eigenvalues of a tensor, and where a thresehold $\epsilon$ (equal to a fraction of the Young's modulus) has been introduced.

\subsubsection{Onset of coalescence for non-local models}

The second criterion is based on the onset of coalescence in order to adjust the physical damage localisation with the numerical one. Thereby, the fast damage growth related to the coalescence phase induces the localisation of damage process inside a band and initiates the cracking process. The use of a non-local model allows the insertion of the cohesive 
band after the onset of softening has been detected, as an approximation, by substituting the derivatives $\frac{\partial \mathbf{P}}{\partial \mathbf{F}}$ in the criterion (67) by $\frac{\partial \mathbf{P}}{\partial \mathbf{F}}+\frac{\partial \mathbf{P}}{\partial \breve{f}_{\mathrm{V}}} \frac{\partial f_{\mathrm{V}}}{\partial \mathbf{F}}$. The condition needs also to carry a directional component. The coalescence condition (35) is thus rewritten in terms of the interface orientation $\boldsymbol{n}_{\mathrm{I}}$ and the Kirchhoff stress tensor (since both are defined in the current configuration, and since $\boldsymbol{\kappa}$ shares the same invariants as $\boldsymbol{\tau}$ ) as

$$
\boldsymbol{n}_{\mathrm{I}} \cdot \boldsymbol{\kappa} \cdot \boldsymbol{n}_{\mathrm{I}}-C_{\mathrm{T}}^{\phi} \tau_{\mathrm{Y}}>0
$$

\subsection{Effects of the cohesive band thickness}

The band thickness directly impacts the energy dissipated during crack propagation. Indeed, at this point, the damage accumulation concentrates inside the band while the surrounding bulk material is elastically unloading. As the main contribution results from the jump opening, the dissipated energy is proportional to $h_{\mathrm{b}}$.

The thickness $h_{\mathrm{b}}$ can be calibrated by comparison of the dissipated energy or $G_{\mathrm{c}}$ with a reference case (Leclerc et al., 2018). This reference could be the underlying non-local model, based on another approach as a phase field model or experimental data. The band thickness can also be linked to a microstructure characteristic length: the coalescence process leads to the concentration of the damage process in a few voids thick layer. This effect will be studied in Section 5.

\section{Numerical implementation}

The implementation of the damage to crack transition inside a discontinuous Galerkin framework is explained in this section. The weak form and its discretised version are derived from the differential partial equations and constitutive models defined in Sections 2 and 3. The implementation and the time integration algorithm for the finite element discretisation and the constitutive models are also described.

\subsection{Discontinuous Galerkin framework}

The discontinuous Galerkin method is similar to other continuous Galerkin finite element methods. The main difference lies in the continuity between elements: the continuity of the approximation is weakly ensured in the former instead of being strongly enforced by the support of the nodal shape functions in the latter. The framework is obtained by following classical derivations for non-linear elliptic equations. The material body is divided into polynomial elements wherein the support of the nodal shape functions is limited to the element they belong to. The interface elements are inserted between those bulk elements at the beginning. The continuity across them is enforced before failure by penalty, symmetrisation and consistency terms. At the transition point, the cohesive term replaces the DG interface terms. Cracks are therefore easily inserted by taking advantage of the dormant discontinuities between elements and do not hurt the natural scalability of the DG method. Furthermore, even though the cohesive band model can be included in any others crack insertions framework, the present DG method already includes integration points at the interface, avoiding any variables interpolation at the crack insertion. 
The formulations presented by Noels and Radovitzky (2006); Wu et al. (2014); Leclerc et al. (2018) are taken as guidelines to develop the CDM/CBM framework to large displacements and plasticity. The result obtained in the following lines is implemented in parallel using the face-based ghost element implementation, following the work of $\mathrm{Wu}$ et al. (2013), inside Gmsh (Geuzaine and Remacle, 2009).

\subsubsection{Strong form of the equations}

The time evolution of the material volume $\Omega$ corresponds to the knowledge of the field variables $(\boldsymbol{u}(\boldsymbol{X}, t), \tilde{Z}(\boldsymbol{X}, t))$. In particular, this includes the location of discontinuity surfaces through time.

The exact solution of the problem $\left(\boldsymbol{u}^{\text {exact }}, \tilde{Z}^{\text {exact }} ; \mathbf{P}^{\text {exact }}, \nabla_{0} \tilde{Z}^{\text {exact }}\right)$ satisfies the system of partial differential equations (2) and (5) and their boundary conditions (3) and (6) over the body $\Omega_{0} \backslash \Gamma_{\mathrm{I} 0}$ described in Section 2. Besides, on the uncracked surfaces $\Gamma_{\mathrm{I} 0}^{\mathrm{U}}$, the solution has to be continuous, i.e.

$$
\llbracket \boldsymbol{u}^{\text {exact }} \rrbracket=\mathbf{0}, \llbracket \mathbf{P}^{\text {exact }} \rrbracket=\mathbf{0}, \llbracket \tilde{Z}^{\text {exact }} \rrbracket=0, \llbracket \mathbf{C}_{1} \cdot \nabla_{0} \dot{\tilde{Z}}^{\text {exact }} \rrbracket=\mathbf{0} \text { on } \Gamma_{\mathrm{I} 0}^{\mathrm{U}} .
$$

On the cracked surfaces $\Gamma_{\text {I0 }}^{\mathrm{C}}$, the relationships (53)-(54) govern the field evolution across the discontinuity. The traction forces are obtained via the cohesive band model described in Section 3. The switch from uncracked to cracked interfaces occurs wherever either the criterion (67) -in the context of a local model- or (68) -in the context of a non-local modelis fulfilled.

\subsubsection{Weak form of the equations}

The initial body $\Omega_{0}$, presented in Sections 2 and 3, is now approximated by a collection of finite volume elements $B^{e}$ with a boundary $S^{e}$ such as $\cup_{e} B^{e} \simeq \Omega_{0}$. In the discontinuous Galerkin framework, the sought approximation for $(\boldsymbol{u}, \dot{\tilde{Z}})$ is element-wise continuous and discontinuous across them, i.e. $(\boldsymbol{u}, \dot{\tilde{Z}}) \in \mathcal{H}^{1}\left(B^{e}\right)$ and $(\boldsymbol{u}, \dot{\tilde{Z}}) \in \mathcal{L}^{2}\left(\Omega_{0}\right)$. The test functions $\left(\boldsymbol{w}_{\boldsymbol{u}}, w_{\tilde{Z}}\right)$ share the same properties. The potential crack surface locations $\Gamma_{\text {I0 }}$ are therefore no longer totally arbitrary but have to follow internal element boundaries, i.e. $\Gamma_{\mathrm{I} 0}=\cup_{e} S^{e} \backslash \Gamma_{0}$. An interface element $S^{s}$ is inserted on the common boundary surface $S^{s}=S^{e+} \cap S^{e-}$ between each pair of elements (arbitrary called $B^{e+}$ and $B^{e-}$ ).

In order to determine the weak form, a similar procedure as in the continuous Galerkin approach is applied. The differential system of the strong form is satisfied in a weightedaverage sense by multiplying it with the test functions and integrating by parts. However, the integration is operated not on the whole domain, but, due to the discontinuities, element by element. One thus has

$$
\begin{aligned}
& \sum_{e} \int_{B^{e}} \rho_{0} \boldsymbol{w}_{\boldsymbol{u}} \cdot \ddot{\boldsymbol{u}} d V_{0}+\sum_{e} \int_{B^{e}} \mathbf{P}: \boldsymbol{\nabla}_{0} \boldsymbol{w}_{\boldsymbol{u}} d V_{0}+\sum_{s} \int_{S^{s} \subset \Gamma_{\mathrm{I} 0}^{\mathrm{C}}} \llbracket \boldsymbol{w}_{\boldsymbol{u}} \rrbracket \cdot\left\langle\mathbf{P}_{\mathrm{b}}\right\rangle \cdot \boldsymbol{N}_{\mathrm{I}}^{-} d S_{0} \\
+ & \sum_{s} \int_{S^{s} \subset \Gamma_{\mathrm{I} 0}^{\mathrm{U}}} \llbracket \boldsymbol{w}_{\boldsymbol{u}} \rrbracket \cdot\langle\mathbf{P}\rangle \cdot \boldsymbol{N}_{\mathrm{I}}^{-} d S_{0}=\sum_{e} \int_{B^{e}} \rho_{0} \boldsymbol{w}_{\boldsymbol{u}} \cdot \boldsymbol{b}_{0} d V_{0}+\sum_{s} \int_{S^{s} \subset \Gamma_{\mathrm{N} 0}} \overline{\boldsymbol{t}_{0}} \cdot \boldsymbol{w}_{\boldsymbol{u}} d S_{0} ;
\end{aligned}
$$




$$
\begin{gathered}
\sum_{e} \int_{B^{e}} \dot{\tilde{Z}} w_{\tilde{Z}} d V_{0}+\sum_{e} \int_{B^{e}} \nabla_{0} w_{\tilde{Z}} \cdot \mathbf{C}_{l} \cdot \nabla_{0} \dot{\tilde{Z}} d V_{0} \\
+\sum_{s} \int_{S^{s} \subset \Gamma_{\mathrm{I} 0}^{\mathrm{U}}} \llbracket w_{\tilde{Z}} \rrbracket\left\langle\mathbf{C}_{l} \cdot \nabla_{0} \dot{\tilde{Z}}\right\rangle \cdot \mathbf{N}_{\mathrm{I}}^{-} d S_{0}=\sum_{e} \int_{B^{e}} \dot{Z} w_{\tilde{Z}} d V_{0} .
\end{gathered}
$$

This element-wise integration introduces a supplementary flux term on the uncracked interfaces, so-called consistency term as it is necessary to obtain a consistent method. As neither the continuity between uncracked elements nor the method stability is enforced, compatibility equations $\boldsymbol{u}^{+}-\boldsymbol{u}^{-}=\mathbf{0}$ and $\dot{\tilde{Z}}^{+}-\dot{\tilde{Z}}^{-}=0$ are weakly added through a penalty term, following the interior penalty method. Furthermore, a symmetrisation term is inserted in the weak form at the uncracked interface to ensure an optimal convergence rate in respect to the mesh size ${ }^{4}$. The weak formulation is finally summarised as finding a solution $(\boldsymbol{u}, \dot{\tilde{Z}})$ such that

$$
\begin{gathered}
\int_{\Omega_{0}} \rho_{0} \boldsymbol{w}_{\boldsymbol{u}} \cdot \ddot{\boldsymbol{u}} d V_{0}+\int_{\Omega_{0}} \mathbf{P}: \boldsymbol{\nabla}_{0} \boldsymbol{w}_{\boldsymbol{u}} d V_{0}+\int_{\Gamma_{\mathrm{I} 0}^{\mathrm{C}}} \llbracket \boldsymbol{w}_{\boldsymbol{u}} \rrbracket \cdot\left\langle\mathbf{P}_{\mathrm{b}}\right\rangle \cdot \boldsymbol{N}_{\mathrm{I}}^{-} d S_{0} \\
\quad+\int_{\Gamma_{\mathrm{I}}^{\mathrm{U}}} \llbracket \boldsymbol{w}_{\boldsymbol{u}} \rrbracket \cdot\langle\mathbf{P}\rangle \cdot \boldsymbol{N}_{\mathrm{I}}^{-} d S_{0}+\int_{\Gamma_{\mathrm{I}}^{\mathrm{U}}} \llbracket \boldsymbol{w}_{\boldsymbol{u}} \rrbracket \otimes \boldsymbol{N}_{\mathrm{I}}^{-}:\left\langle\frac{\beta_{s}}{h_{s}} \mathcal{C}^{\mathrm{e}}\right\rangle: \llbracket \boldsymbol{u} \rrbracket \otimes \boldsymbol{N}_{\mathrm{I}}^{-} d S_{0} \\
\quad+\int_{\Gamma_{\mathrm{I} 0}^{\mathrm{U}}} \llbracket \boldsymbol{u} \rrbracket \cdot\left\langle\mathcal{C}^{\mathrm{e}}: \boldsymbol{\nabla}_{0} \boldsymbol{w}_{\boldsymbol{u}}\right\rangle \cdot \boldsymbol{N}_{\mathrm{I}}^{-} d S_{0}=\int_{\Omega_{0}} \rho_{0} \boldsymbol{w}_{\boldsymbol{u}} \cdot \boldsymbol{b}_{0} d V_{0}+\int_{\Gamma_{\mathrm{N} 0}} \overline{\boldsymbol{t}_{0}} \cdot \boldsymbol{w}_{\boldsymbol{u}} d S_{0} ; \\
\quad \int_{\Omega_{0}}\left(\dot{\tilde{Z}} w_{\tilde{Z}}+\nabla_{0} w_{\tilde{Z}} \cdot \mathbf{C}_{\mathrm{l}} \cdot \boldsymbol{\nabla}_{0} \dot{\tilde{Z}}\right) d V_{0}+\int_{\Gamma_{\mathrm{I} 0}^{\mathrm{U}}} \llbracket w_{\tilde{Z}} \rrbracket\left\langle\mathbf{C}_{\mathrm{l}} \cdot \boldsymbol{\nabla}_{0} \dot{\tilde{Z}}\right\rangle \cdot \boldsymbol{N}_{\mathrm{I}}^{-} d S_{0} \\
+\int_{\Gamma_{\mathrm{I} 0}^{\mathrm{U}}} \llbracket w_{\tilde{Z}} \rrbracket \boldsymbol{N}_{\mathrm{I}}^{-} \cdot\left\langle\frac{\beta_{s}}{h_{s}} \mathbf{C}_{\mathrm{l}}\right\rangle \cdot \boldsymbol{N}_{\mathrm{I}}^{-} \llbracket \dot{\tilde{Z}} \rrbracket d S_{0}+\int_{\Gamma_{\mathrm{I} 0}^{\mathrm{U}}} \llbracket\left\langle\mathbf{C}_{l} \cdot \nabla_{0} w_{\tilde{Z}}\right\rangle \cdot \boldsymbol{N}_{\mathrm{I}}^{-} d S_{0} \\
=\int_{\Omega_{0}} \dot{Z} w_{\tilde{Z}} d V_{0},
\end{gathered}
$$

for $\forall\left(\boldsymbol{w}_{\boldsymbol{u}}, w_{\tilde{Z}}\right)$ kinematically admissible. It introduces $\mathcal{C}^{\mathrm{e}}=\left.\frac{\partial \mathbf{P}}{\partial \mathbf{F}}\right|_{\mathbf{F}=\mathbf{I}}$ the constant elastic operator of the virgin material, $\beta_{s}>1$, a sufficiently high penalty parameter for solution stabilisation, and $h_{s}$, a characteristic mesh size parameter dependent on the element size and the involved polynomial approximation.

\subsubsection{Finite element discretisation}

The weak form (72)-(73) developed in the previous section is the starting point of the finite element discretisation. Inside each element, a nodal interpolation approximates the fields $(\boldsymbol{u}, \dot{\tilde{Z}})$ and their respective test functions $\left(\boldsymbol{w}_{\boldsymbol{u}}, w_{\tilde{Z}}\right)$. At each node $a$ is associated one polynomial shape function $N^{a}(\boldsymbol{X})$, identical for each field and test function. The fields (and

\footnotetext{
${ }^{4}$ Optimal convergence rate can only be demonstrated in the linear range for history-dependent materials.
} 
the test functions) are therefore estimated inside an element following:

$$
\begin{array}{ll}
\boldsymbol{u}(\boldsymbol{X})=\sum_{a=1}^{N_{\mathrm{n}}} N^{a}(\boldsymbol{X}) \boldsymbol{u}^{a}, & \Delta \tilde{Z}(\boldsymbol{X})=\sum_{a=1}^{N_{\mathrm{n}}} N^{a}(\boldsymbol{X}) \Delta \tilde{Z}^{a}, \quad \text { and } \\
\boldsymbol{w}_{\boldsymbol{u}}(\boldsymbol{X})=\sum_{a=1}^{N_{\mathrm{n}}} N^{a}(\boldsymbol{X}) \delta \boldsymbol{u}^{a}, & w_{\tilde{Z}}(\boldsymbol{X})=\sum_{a=1}^{N_{\mathrm{n}}} N^{a}(\boldsymbol{X}) \delta \tilde{Z}^{a},
\end{array}
$$

from the $N_{\mathrm{n}}$ nodal values $\left(\boldsymbol{u}^{a}, \tilde{Z}^{a}\right)$ (and $\left(\delta \boldsymbol{u}^{a}, \delta \tilde{Z}^{a}\right)$ ) associated with each of the $N_{\mathrm{n}}$ nodes of the element. The degrees of freedom (DoF) are brought together in a nodal unknowns vector $\boldsymbol{q}^{a}=\left[\left(\boldsymbol{u}^{a}\right)^{\mathrm{T}} \Delta \tilde{Z}^{a}\right]^{\mathrm{T}}$ for a node $a$ and in a global vector $\boldsymbol{q}=\left[\boldsymbol{u}^{\mathrm{T}} \Delta \tilde{\boldsymbol{Z}}^{\mathrm{T}}\right]^{\mathrm{T}}$ gathering all the DoF of the mesh. Applying to the weak form (72)-(73) the discrete approximation (74) of the different fields, the following set of differential equations is obtained by taking into account the inter-element discontinuities

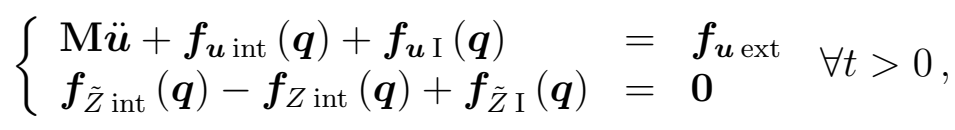

see Appendix D for details and expressions. In the force equilibrium equations $(75), \boldsymbol{f}_{\boldsymbol{u} \text { int }}$, $\boldsymbol{f}_{\boldsymbol{u} \text { I }}$ and $\boldsymbol{f}_{\boldsymbol{u} \text { ext }}$ respectively stand for the internal, interface and external force vectors related to the displacement field while $\mathbf{M}$ is the mass matrix. $\boldsymbol{f}_{\tilde{Z} \text { int }}, \boldsymbol{f}_{Z \text { int }}$ and $\boldsymbol{f}_{\tilde{Z} \text { I }}$ are respectively the non-local internal, local internal and interface non-local force vectors.

\subsection{Numerical time integration}

The set of equations (75) is integrated through time using the $\alpha$-generalised method developed by Chung and Hulbert (1993), see the Figure 8. Despite the fact that the problem is highly non-linear, an implicit monolithic scheme has been chosen at the level of the global fields in combination to the implicit integration scheme at the level of the constitutive material law, presented in Section 2.4. This combination has been chosen to keep the residues, or the error, under control. Moreover, the scheme is unconditionally stable, allowing large time steps and mitigating the large CPU cost of the material law integration. Besides, the scheme has a supra-linear convergence (almost two near the solution) as the material tensor operators and stiffness matrices are evaluated analytically. However, soon after crack insertion, the convergence rate tends to decrease. This effect is mitigated by using a dynamic implicit scheme instead of a more simple quasi-static scheme since the dynamic effects have a stabilising effect on the solution: they ease, for instance, to overcome local snap backs.

Practically, the unknowns at time $t^{n+1}$ and their time derivatives are approximated following

$$
\begin{aligned}
\boldsymbol{q}\left(t^{n+1}\right) & =\boldsymbol{q}\left(t^{n}\right)+\Delta t \dot{\boldsymbol{q}}\left(t^{n}\right)+\left[\frac{1}{2}-\beta\right] \Delta t^{2} \ddot{\boldsymbol{q}}\left(t^{n}\right)+\beta \Delta t \ddot{\boldsymbol{q}}\left(t^{n+1}\right) \\
\dot{\boldsymbol{q}}\left(t^{n+1}\right) & =\dot{\boldsymbol{q}}\left(t^{n}\right)+[1-\gamma] \Delta t \ddot{\boldsymbol{q}}\left(t^{n}\right)+\gamma \Delta t \ddot{\boldsymbol{q}}\left(t^{n+1}\right)
\end{aligned}
$$

introducing $\beta$ and $\gamma$ as Newmark integration parameters and $\Delta t=t^{n+1}-t^{n}$ as the time step. From the force balance system of Eq. (75) weighted at time $t^{n}$ and $t^{n+1}$, a residual form $\boldsymbol{r}\left(\boldsymbol{q}^{(k)}\left(t^{n+1}\right)\right)$ is obtained as 


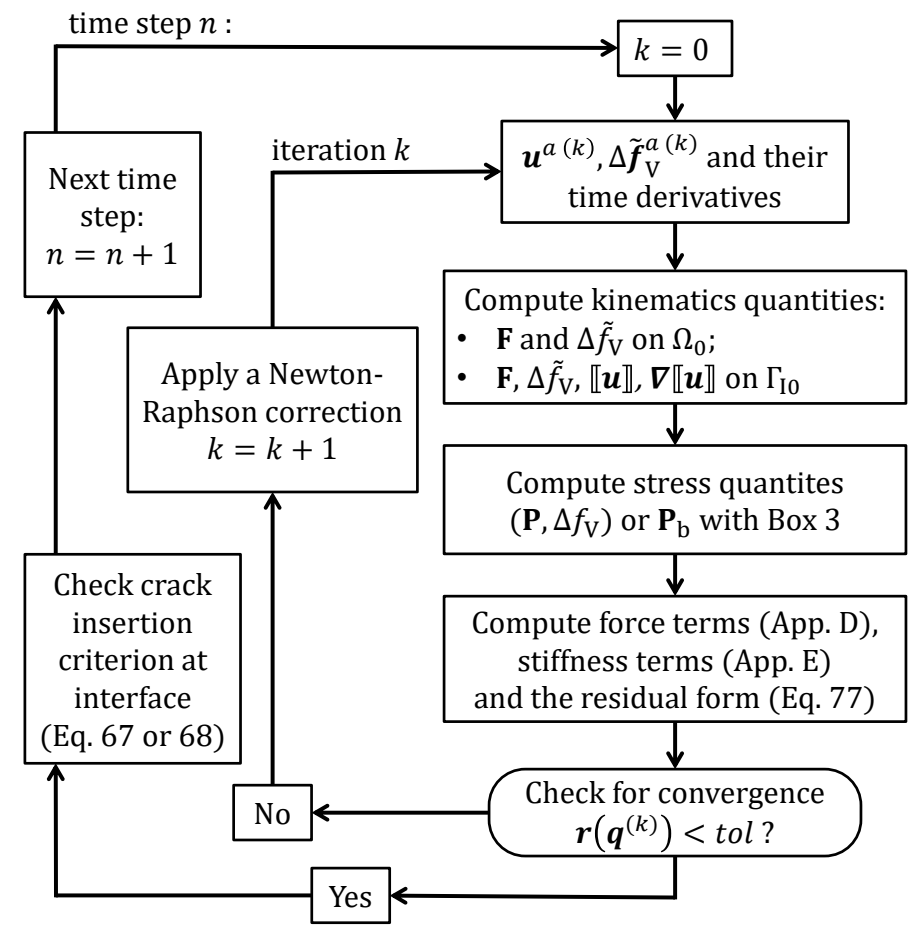

Figure 8: The implicit monolithic scheme structure using the $\alpha$-generalised method.

$$
\begin{aligned}
\boldsymbol{r}\left(\boldsymbol{q}^{(k)}\left(t^{n+1}\right), t^{n+1}\right) & =\left[1-\alpha_{\mathrm{M}}\right]\left[\begin{array}{cc}
\mathbf{M} & \mathbf{0} \\
\mathbf{0} & \mathbf{0}
\end{array}\right] \ddot{\boldsymbol{q}}^{(k)}\left(t^{n+1}\right)+\alpha_{\mathrm{M}}\left[\begin{array}{cc}
\mathbf{M} & \mathbf{0} \\
\mathbf{0} & \mathbf{0}
\end{array}\right] \ddot{\boldsymbol{q}}\left(t^{n}\right), \\
& +\left[1-\alpha_{\mathrm{F}}\right] \boldsymbol{f}_{\boldsymbol{q}}\left(\boldsymbol{q}^{(k)}\left(t^{n+1}\right)\right)+\alpha_{\mathrm{F}} \boldsymbol{f}_{\boldsymbol{q}}\left(\boldsymbol{q}\left(t^{n}\right)\right)
\end{aligned}
$$

where $\boldsymbol{q}^{(k)}\left(t^{n}\right)$ is the nodal values vector of the field at iteration $k$ and at time step $t^{n}$. The parameter $\alpha_{\mathrm{M}}$ weights the inertia forces expressed at time $t^{n+1}$ and $t^{n}$ while $\alpha_{\mathrm{F}}$ scales the sum of the internal, external and interface force terms gathered in

$$
\boldsymbol{f}_{\boldsymbol{q}}(\boldsymbol{q}(t))=\left[\begin{array}{c}
\boldsymbol{f}_{\boldsymbol{u} \text { int }}(\boldsymbol{q}(t))+\boldsymbol{f}_{\boldsymbol{u} \mathrm{I}}(\boldsymbol{q}(t))-\boldsymbol{f}_{\boldsymbol{u} \text { ext }}(t) \\
\boldsymbol{f}_{\tilde{Z} \text { int }}(\boldsymbol{q}(t))-\boldsymbol{f}_{Z \text { int }}(\boldsymbol{q}(t))+\boldsymbol{f}_{\tilde{Z} \mathrm{I}}(\boldsymbol{q}(t))
\end{array}\right] .
$$

The correction is computed by solving the system

$$
\mathbf{S}\left(\boldsymbol{q}^{(k)}\left(t^{n+1}\right), t^{n+1}\right) \Delta \boldsymbol{q}^{(k+1)}\left(t^{n+1}\right)=-\boldsymbol{r}\left(\boldsymbol{q}^{(k)}\left(t^{n+1}\right), t^{n+1}\right),
$$

until convergence is reached. In Eq. (79), the tangent matrix $\mathbf{S}$ reads

$$
\mathbf{S}=\frac{\partial \boldsymbol{r}}{\partial \boldsymbol{q}}=\left[1-\alpha_{\mathrm{M}}\right] \frac{1}{\beta \Delta t^{2}}\left[\begin{array}{cc}
\mathbf{M} & \mathbf{0} \\
\mathbf{0} & \mathbf{0}
\end{array}\right]+\left[1-\alpha_{\mathrm{F}}\right] \mathbf{K}
$$

where the stiffness matrix $\mathbf{K}$ is detailed in Appendix E. The values of the four integration parameters are determined in terms of the (infinite) spectral radius $\rho_{\infty}$ in the work of Chung and Hulbert (1993). 


\subsection{Specific details of the algorithm}

This section gathers some practical details of the numerical algorithm, contributing to the framework robustness.

- A crack surface is introduced at a whole interface element when the criterion is detected at least at one of its Gauss points. Void evolution is also blocked on the neighbouring volume elements (but not the plastic flow), in order to favor elastic unloading instead of further volume softening.

- In order to avoid inter-element penetration during crack opening, a (linear) penalty force is added. However, no friction relation is taken into account. Furthermore, the normal part contribution of the jump to $\mathbf{F}_{\mathrm{b}}$ in Eq. (61) is limited in the case of negative values to avoid negative Jacobian value.

- At a crack insertion using the coupled model presented in Section 2.3.5, a small shift exits between the criterion (68) and the yield surface (38). To avoid stress discontinuity, an offset is therefore introduced in Eq. (38).

- The number of simultaneous crack insertions is limited at each sub-step, without inhibiting crack branching.)

\section{Numerical applications}

The constitutive description of the ductile failure process and its numerical implementation is applied in this section to some numerical examples. In order to verify the formulation, the problem analysed by Huespe et al. (2012), in which a crack is inserted at loss of ellipticity of a local model and in which the crack opening is governed by an adequate calibration of the Gurson model parameters, is first reproduced. The effects of the band thickness and the non-dependence to the mesh size are analysed. Then, the more physical Thomason coalescence criterion is used as crack initiation criterion and the crack opening is governed by the Thomason coalescence model. It is shown that, in this case, although cracks are inserted during the strain softening stage of the process, because of the non-local formulation of the damage model, the solution is not mesh dependent. Furthermore, the model is able to reproduce the slant and cup-cone failure modes in plane-strain specimens and axisymmetric bars, either smooth or notched, respectively. Finally, the effect of the non-local characteristic length on the resulting material ductility is studied.

\subsection{Framework verification with a local Gurson model applied on a plane strain specimen}

The current framework is first degenerated into a local Gurson model ${ }^{5}$ to allow a comparison with the results discussed by Huespe et al. (2012). With the local version of the

\footnotetext{
${ }^{5}$ To recover a local model, the non-local framework is used with a small non-local length compared to the mesh size, here equal to $5[\mu \mathrm{m}]$, i.e. one $15^{\text {th }}$ of the finest element size. Moreover, the derivatives $\frac{\partial \mathbf{P}}{\partial \mathbf{F}}$ in the criterion (67) is substituted by $\frac{\partial \mathbf{P}}{\partial \mathbf{F}}+\frac{\partial \mathbf{P}}{\partial \breve{f}_{\mathrm{V}}} \frac{\partial f_{\mathrm{V}}}{\partial \mathbf{F}}$, since $\breve{f}_{\mathrm{V}} \simeq f_{\mathrm{V}}$ in this case.
} 


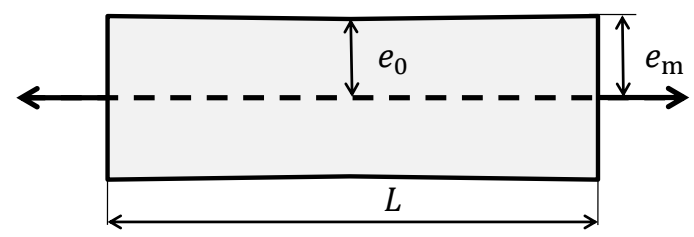

Figure 9: Geometry of the plane strain specimen.

Gurson model, a crack is inserted when the loss of ellipticity is detected by the criterion (67) in order to preserve solution uniqueness. The failure process is governed by the same Gurson model in which coalescence is modelled by adequate parameters. The plane strain specimen consists in a rectangular bar of thickness $2 e_{\mathrm{m}}=5[\mathrm{~mm}]$ and length $L=3.5 e_{\mathrm{m}}$ as drawn on Fig 9. Practically, the problem symmetry is exploited to model only one half of the specimen. To ensure localisation at the centre of the bar, a geometrical defect is introduced. For this purpose, the thickness linearly decreases with a minimum thickness $e_{0}=0.98 e_{\mathrm{m}}$ in the middle of the bar. This smallest cross section is chosen as the reference surface area. The specimen is meshed using 6-node quadratic triangular elements with an aspect ratio between 4 and 5 in the necking zone to ensure a square mesh near failure. Three mesh sizes scaled by a refining factor of 1.5 are used: $l_{\text {mesh }}=75[\mu \mathrm{m}]$ for the finest mesh, $l_{\text {mesh }}=110[\mu \mathrm{m}]$ and $l_{\text {mesh }}=170[\mu \mathrm{m}]$ for the coarsest (and an aspect ratio of 4) corresponding respectively to 65,45 and 30 elements through the thickness, and including respectively 7180, 4224 and 2544 triangles in total. The stability parameter $\beta_{s}$ is fixed to 50 , a sufficiently high value to provide results independent of $\beta_{s}$. As dynamic effects are out of the scope of this work, the spectral radius $\rho_{\infty}$ is set to 0 and the loading time is long enough for the results to become insensitive to them.

The material properties, gathered in Table 1, are similar to the one used by Huespe et al. (2012) and by Besson et al. (2003) ${ }^{6}$. The hardening law consists in a Swift law in terms of the matrix plastic strain

$$
\tau_{\mathrm{Y}}(\hat{p})=\tau_{\mathrm{Y} 0}\left(1+h_{\mathrm{y}} \hat{p}\right)^{h_{\exp }} .
$$

The strain-controlled nucleation term is a Gaussian function (Chu and Needleman, 1980)

$$
A_{\text {nucl }}=\frac{f_{\mathrm{n}}}{s_{\mathrm{n}} \sqrt{2 \pi}} \exp \left[-\frac{1}{2}\left(\frac{\hat{p}-p_{\mathrm{n}}}{s_{\mathrm{n}}}\right)^{2}\right],
$$

where $f_{\mathrm{n}}, p_{\mathrm{n}}$ and $s_{\mathrm{n}}$ are distribution parameters for the nucleated void part identically to Huespe et al. (2012). The shear contribution from $k_{\omega}$ is also ignored. A scatter of $1 \%$ is added to the initial porosity $f_{\mathrm{V}_{0}}=1.5 \times 10^{-4}$ in order to break the symmetry in the problem. The local Gurson model parameters are $q_{1}=q_{3}=1.5 ; q_{2}$ is equal to 1.15 to simulate the coalescence similarly to Besson et al. (2003). The crack is introduced when the criterion (67), based on the loss of ellipticity, is satisfied. The band thickness is at first fixed to

\footnotetext{
${ }^{6}$ We refer to so-called "G-model" considered in this last reference
} 
Table 1: Material properties for the local Gurson model, adapted from Huespe et al. (2012).

Elasto-plastic properties, Eqs. (21), (81):

\begin{tabular}{lcl} 
Density & $\rho_{0}$ & $7600\left[\mathrm{~kg} / \mathrm{m}^{3}\right]$ \\
Young's Modulus & $E$ & $210[\mathrm{GPa}]$ \\
Poisson ratio & $\nu$ & 0.3 \\
Initial yield stress & $\tau_{\mathrm{Y} 0}$ & $377[\mathrm{MPa}]$ \\
Strain-hardening modulus & $h_{\mathrm{y}}$ & 555 \\
Strain-hardening exponent & $h_{\exp }$ & 0.12 \\
\hline Porosity parameters, Eqs.(26), (82): & & \\
Initial porosity & $f_{\mathrm{V}_{0}}$ & $1.5 \times 10^{-4}$ \\
Nucleation parameters & $f_{\mathrm{n}}$ & $4.0 \times 10^{-6}$ \\
& $p_{\mathrm{n}}$ & 0.3 \\
& $s_{\mathrm{n}}$ & 0.1 \\
Shear-induced parameter & $k_{\omega}$ & 0 \\
\hline Gurson model parameters, Eq.(32): & & \\
Gurson yield parameters & $q_{1}, q_{3}$ & 1.5 \\
& $q_{2}$ & 1.15 \\
\hline Cohesive band model: & & \\
Cohesive band thickness & $h_{\mathrm{b}}$ & $12[\mu \mathrm{m}]$
\end{tabular}

$12[\mu \mathrm{m}]$, slightly higher than Huespe et al. (2012) since these authors have considered an exponential smoothing of the porosity evolution contrarily to the present analysis.

The force evolution is represented in Fig. 10 as a function of the thickness reduction $\Delta e / e_{0}$ for the three mesh sizes. At first, on Fig. 10(a), the results are shown for the local model without crack insertion. In this case, the results are mesh-dependent as expected. However, on Fig. 10(b), solutions involving the insertion of the cohesive band model (continuous lines) at loss of ellipticity are mesh independent during the entire deformation process. Except for the coarsest mesh, characterised by too large elements to correctly model the problem, only small disparities are observed during crack propagation, essentially due to different crack paths that are constrained along the interface elements. Moreover, the results are globally in agreement with the ones obtained by Huespe et al. (2012).

The corresponding plastic strain and the porosity distributions (on a logarithmic scale) of the local Gurson model coupled with the CBM are presented in Figs. 11 and 12 after the first crack insertion, during crack propagation, and at complete failure for the finest mesh. Two shear bands oriented at around $45^{\circ}$, noticeable by a higher plastic strain or a higher porosity value, intersect at the centre of the specimen. The crack initiates at the centre and spreads toward the surface, following one of those shear bands. While the coarsest mesh is again not refined enough to correctly model the problem, resulting in a crack orientation more vertical than expected, the crack orientation is around $45^{\circ}$ for the other two meshes. We however note that the crack eventually bifurcates towards the secondary shear band direction as both localisation directions are possible. This behaviour was prevented in the 


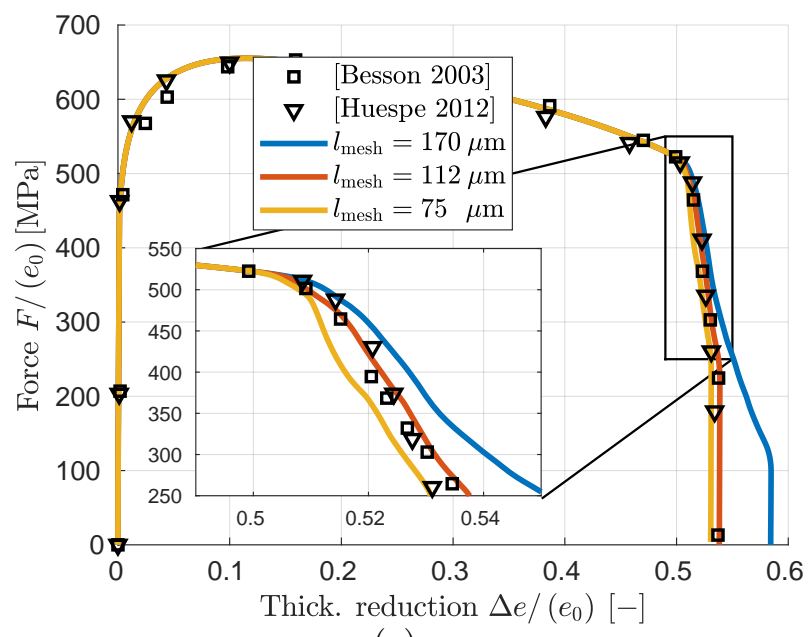

(a)

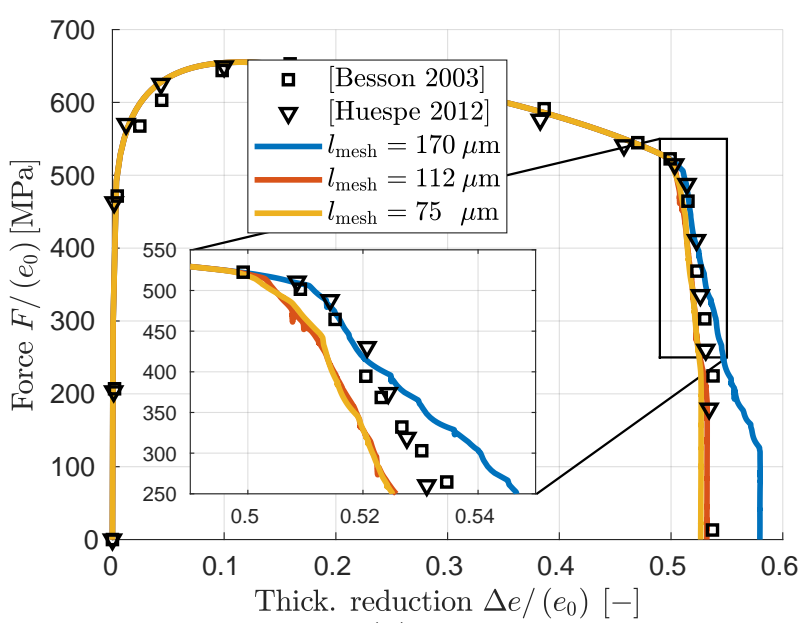

(b)

Figure 10: Variation of the applied load as a function of minimum cross-section thickness reduction of the plane strain specimen (a) for the local Gurson model without crack insertion, and (b) for the local Gurson model coupled with the CBM (continuous lines) for the three mesh sizes, and comparison with the results of Besson et al. (2003) and Huespe et al. (2012) (square and triangle makers).

work of Huespe et al. (2012) by systematically favouring one direction. We will further show that when considering a non-local model coupled with the Thomason coalescence criterion this is naturally avoided.

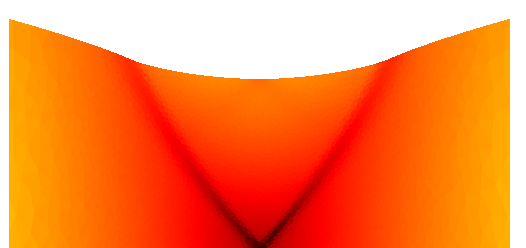

(a)

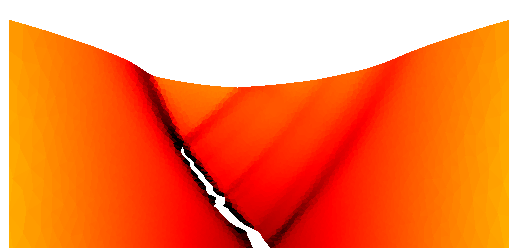

(b)

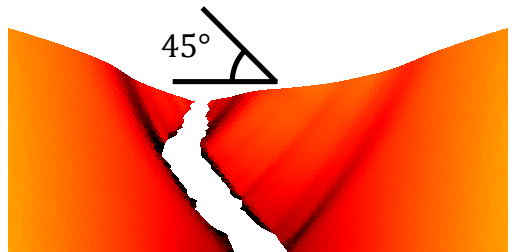

(c)

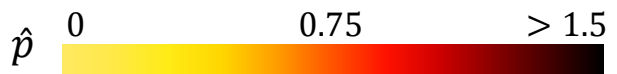

Figure 11: Matrix plastic strain distribution of the plane strain specimen model by introducing a crack at the loss of ellipticity of the local model: (a) at failure initiation, (b) during crack propagation and (c) at total failure for the fine mesh.

The response in terms of the band thickness is now examined in Fig. 13 using the medium mesh $(110[\mu \mathrm{m}])$. If no clear trend is observed on the whole curve, the band thickness clearly influences the response right after the onset of cracking as seen in the zoom: the behaviour is more ductile with a thicker band. However, quickly after the onset of cracking, crack paths slightly differ from one another, resulting in no comparable curves. A proper comparison could be performed by enforcing a pre-defined path. It is worth mentioning that a vanishing value of $h_{\mathrm{b}}$, closer to the expected brittle fracture behaviour, results in a straight crack 


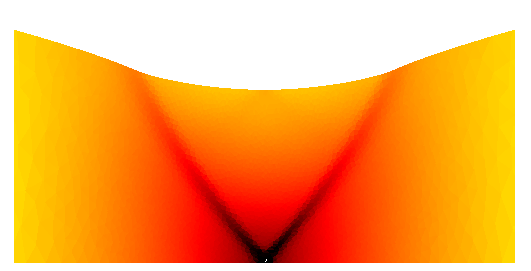

(a)

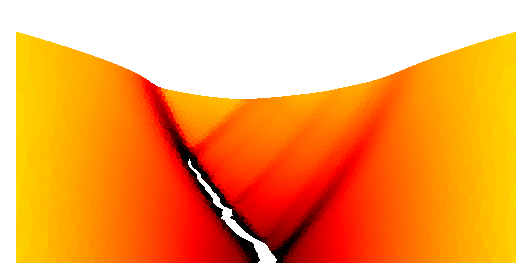

(b)

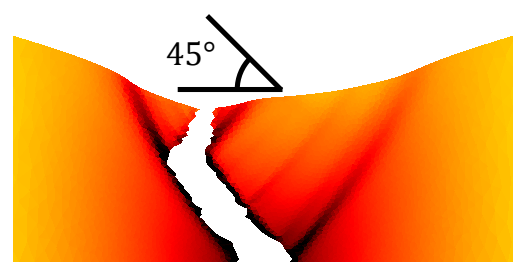

(c)

$$
f_{V} \quad 0.015 \% \quad 0.3 \% \quad>6 \%
$$

Figure 12: Porosity distribution inside the plane strain specimen model by introducing a crack at the loss of ellipticity of the local model: (a) at failure initiation, (b) during crack propagation and (c) at total failure for the fine mesh.

path. Indeed, the plastic strain distribution is compared on Fig. 14 for two other values of $h_{\mathrm{b}}$. In this case, increasing $h_{\mathrm{b}}$ does not change the crack orientation while decreasing it results in a straight crack. Surface energy dissipation, although small compared to the diffuse plastic dissipation, is therefore required to avoid this inconsistency with respect to physical evidences.

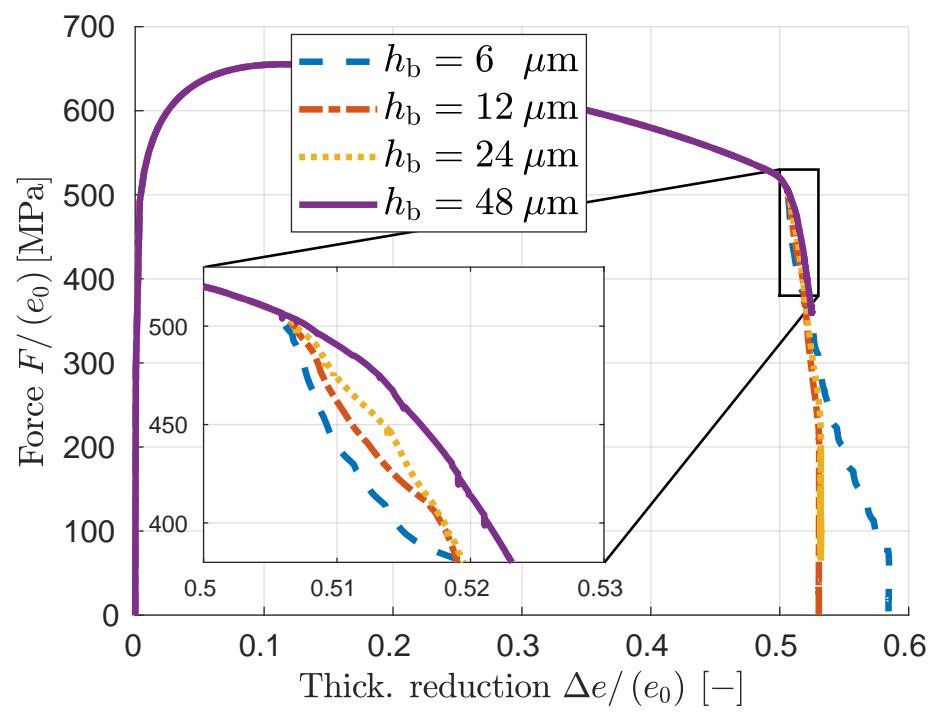

Figure 13: Variation of the applied force as a function of the minimum cross-section thickness reduction of the plane strain specimen for the local Gurson model coupled with the CBM and for different values of the band thickness. A zoom is provided for the response just at and after cracking initiation.

\subsection{Non-local model and crack insertion based on Thomason coalescence model}

The insertion criterion (67) is now replaced by the coalescence criterion (68) inspired from the Thomason coalescence model. This last one has the advantage to rely more on the void geometry evolution. As we will illustrate later, this condition is generally fulfilled 


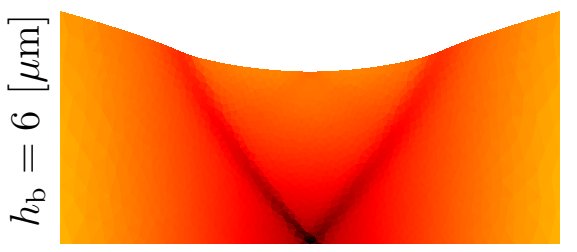

(a)

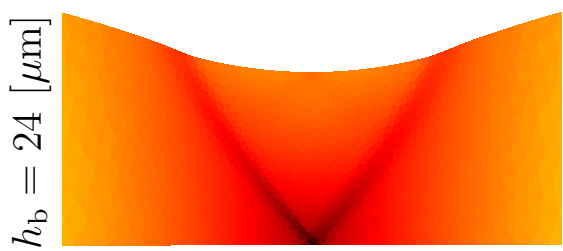

(d)

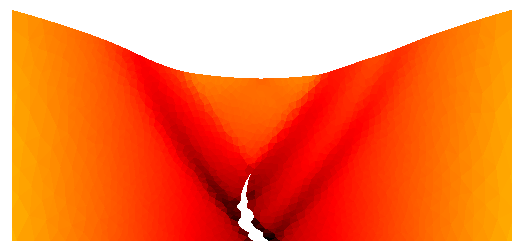

(b)

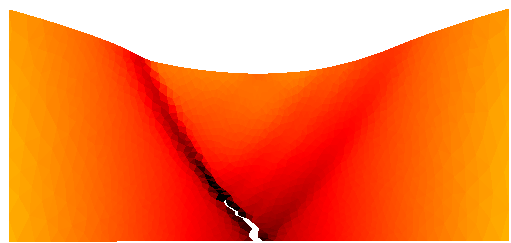

(e)

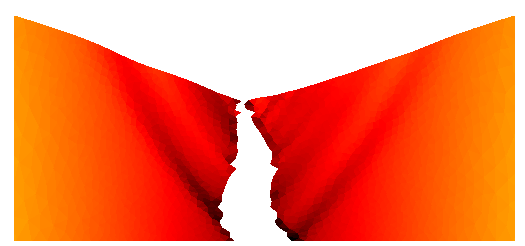

(c)

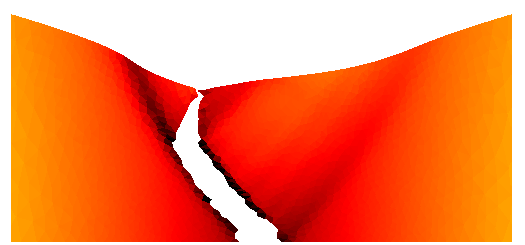

(f)

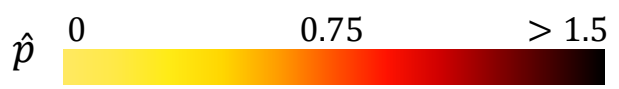

Figure 14: Matrix plastic strain distribution within the plane strain specimen model by introducing a crack at loss of ellipticity of the local model for two values of $h_{\mathrm{b}}$ : (left) at failure initiation, (center) during crack propagation and (right) at total failure for the medium refined mesh.

beyond the softening point, hence the necessity of using the non-local model. After crack insertion, another coalescence model is used to govern the failure process: the coupled Gurson-Thomason model presented in Section 2.3.4. Since the model is different, in this section, the material properties of the Gurson-Thomason combined model are first identified in order to recover the results reported by Besson et al. (2003).

\subsubsection{Parameters calibration}

The elasto-plastic material parameters of Section 5.1 are used again, see Table 1. The other materials parameters are gathered in Table 2. The Gurson parameters are now $q_{1}=$ $q_{3}=1.5$ and $q_{2}=1$, which are more micromechanically sound (see Faleskog et al. (1998)) than the values used in Section 5.1. Two non-local lengths are investigated: 50 and $75[\mu \mathrm{m}]$, which are reasonable values with respect to literature but which are of course microstructure dependent, and will vary from one material to another (e.g. Zhang et al. (2018)). The band thickness is still equal to $h_{\mathrm{b}}=12[\mu \mathrm{m}]$. The ligament ratio $\chi$ is computed using $\lambda_{0}=0.5$ and $\kappa=0.4$, see Eq. (31). In addition, the interpolation exponent $n$ of the yield surface $\phi_{\mathrm{T}}$ is equal to 10 .

The nucleation term $A_{\mathrm{n}}$ and the shear contribution $k_{\omega}$ are then calibrated in terms of the non-local length $l_{\mathrm{c}}=50[\mu \mathrm{m}]$ (resp. $\left.l_{\mathrm{c}}=75[\mu \mathrm{m}]\right)$ to predict the fracture strain value of the smooth round bar and of the plane strain specimen reported by Besson et al. (2003). As $k_{\omega}$ has no effect on failure initiation on the axisymmetric tests, as it will be shown, a significant nucleation term with $f_{\mathrm{n}}=2.0 \times 10^{-3}\left(\right.$ resp. $\left.3.5 \times 10^{-3}\right), p_{\mathrm{n}}=0.1$ and $s_{\mathrm{n}}=0.3$ is added to capture the correct initiation point of this test. Then, an approximate shear contribution, $k_{\omega}=1$ (resp. $k_{\omega}=0.5$ ) in agreement with literature (Xue et al., 2010, e.g.), 
Table 2: Material properties for the non-local Gurson-Thomason model.

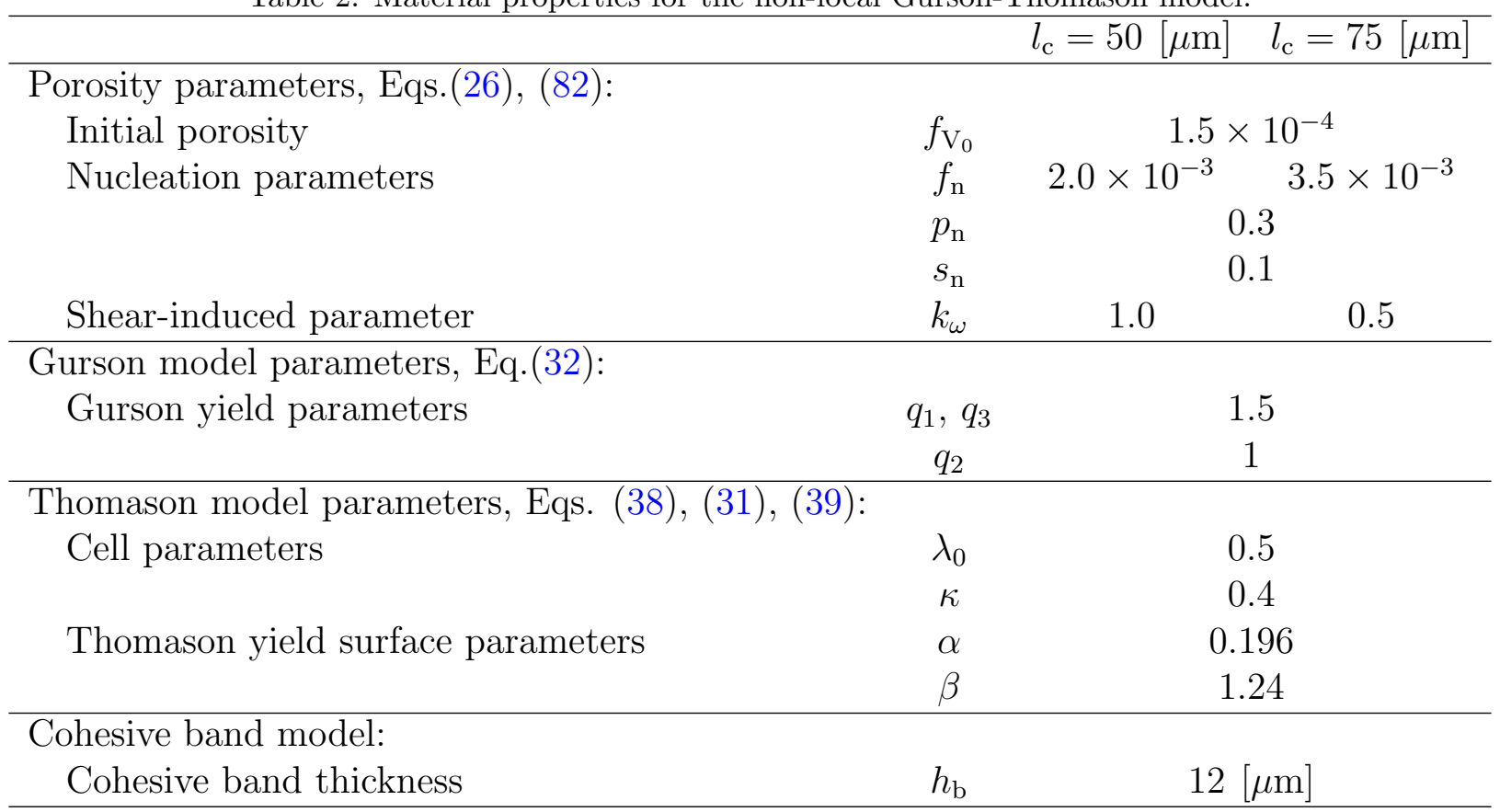

is chosen to fit the fracture initiation of the plane strain specimen.

Unless otherwise stated, the simulations are performed with $l_{\mathrm{c}}=50[\mu \mathrm{m}]$.

\subsubsection{Slant fracture in a plane strain specimens}

The framework is first applied to the plane strain specimen, previously presented in Section 5.1 with $l_{\mathrm{c}}=50[\mu \mathrm{m}]$. Figure $15(\mathrm{a})$ shows the force evolution predicted with the three meshes used earlier. Since the elements are quadratic, the distance $l_{\mathrm{c}}=50 \quad[\mu \mathrm{m}]$ actually covers several integration points for the finest meshes. As observed previously, the simulations with the coarsest mesh do not represent correctly the failure process. Regarding other meshes, the expected necking process is recovered. Moreover, no mesh-dependency is observed in the force-thickness reduction curves.

The plastic strain distribution is represented in Fig. 16 for the medium refined mesh. Before crack insertion, two shear bands are developing at around $45^{\circ}$ with respect to the loading direction at future crack propagation sites. Cracks are then introduced by following one of these localisation bands until total failure. We note that we do not have introduced a direction criterion to select the band direction. This insertion occurs in this case beyond the onset of softening shown by the coloured elements on Fig. 17. The process zone spreads over several elements and precedes the crack tip. While the process zone is almost symmetric at failure initiation, it is afterwards inclined at $45^{\circ}$. 


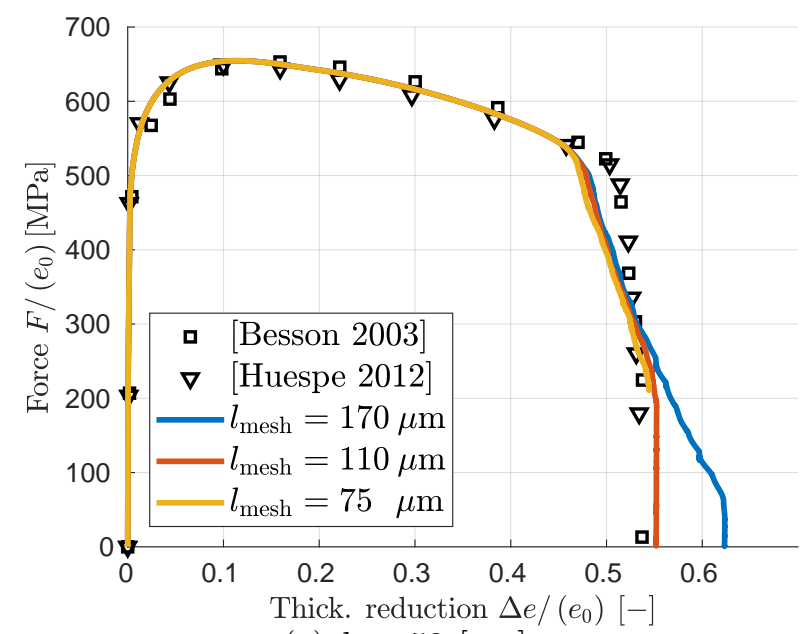

(a) $l_{\mathrm{c}}=50[\mu \mathrm{m}]$

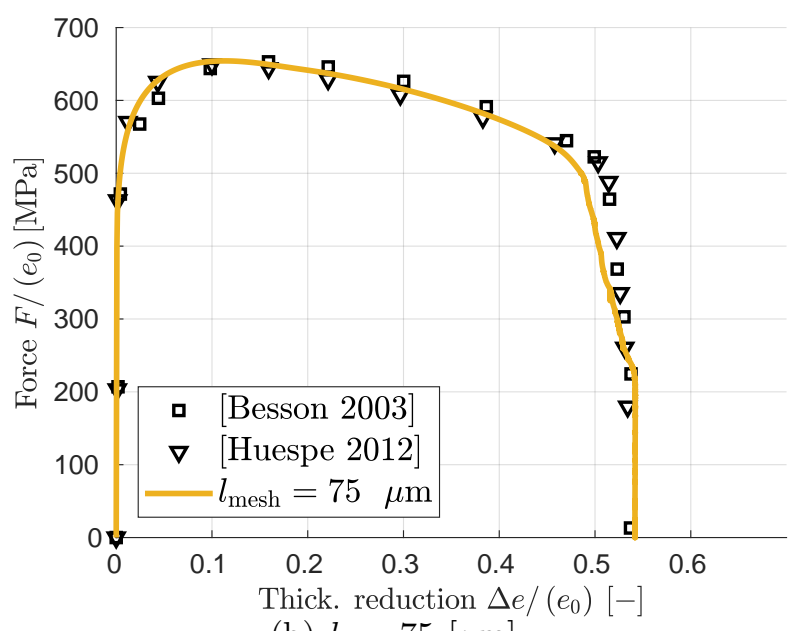

(b) $l_{\mathrm{c}}=75[\mu \mathrm{m}]$

Figure 15: Variation of the applied force as a function of the minimum cross-section thickness reduction of the plane strain specimen for the non-local Gurson-Thomason model coupled with the CBM (continuous lines) for two values of $l_{\mathrm{c}}$, and comparison with the results of Besson et al. (2003) and Huespe et al. (2012) (respectively square and triangle markers).

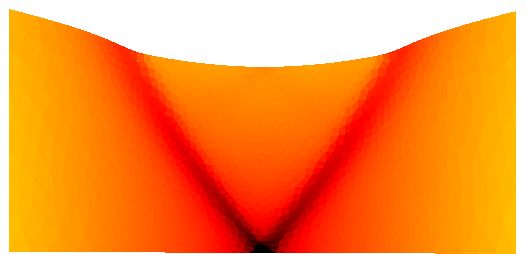

(a)

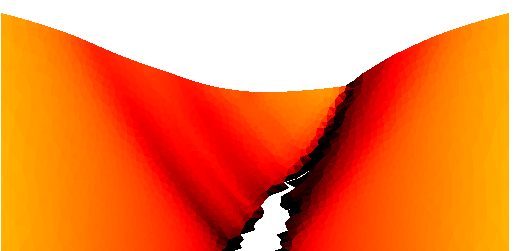

(b)

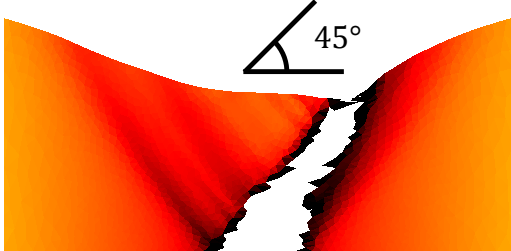

(c)

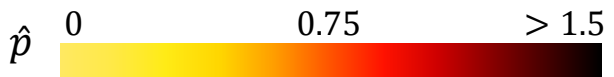

Figure 16: Matrix plastic strain distribution of the plane strain specimen using the non-local model with $l_{\mathrm{c}}=50[\mu \mathrm{m}]$ and introducing a crack following Thomason coalescence criterion and for the medium refined mesh: (a) at failure initiation, (b) during crack propagation and (c) at total failure.

\subsubsection{Cup-cone fracture in round bar specimens}

The framework is now applied to a different stress state by considering axisymmetric smooth and notched round bars, represented respectively in Figs. 18(a) and 18(b). The round specimens have a radius $r_{\mathrm{e}}=5[\mathrm{~mm}]$ and a length $L=3.5 r_{\mathrm{e}}$. The considered notch radius of the notched bars is equal to $r_{\mathrm{n}}=4[\mathrm{~mm}]$ with an external radius $r_{\mathrm{e}}=9[\mathrm{~mm}]$. For the smooth round bar (i.e. $r_{\mathrm{n}}=\infty$ ), a geometrical imperfection is introduced. It consists in a diameter reduction around the central section as applied by Huespe et al. (2012). The reduction follows a circular profile, extending on one seventh of the bar length from both sides of the maximum reduction $r_{0}=0.98 r_{\mathrm{e}}$ section. The minimum cross-section is used as reference surface $S_{0}$. The meshes for the different notch radii $r_{\mathrm{n}}=\infty$ and 4 [mm] consist 


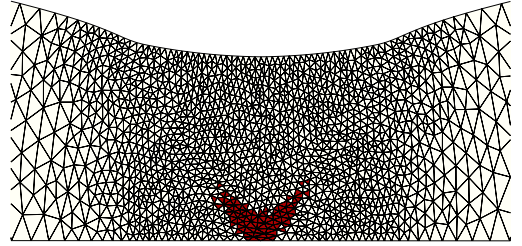

(a)

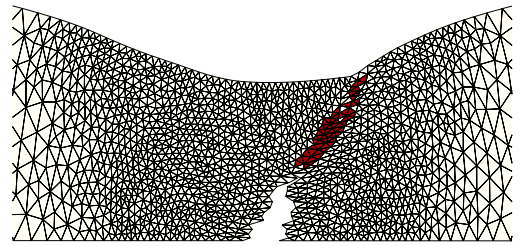

(b)

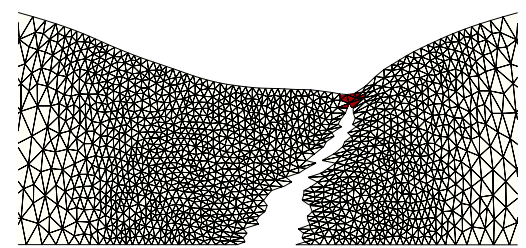

(c)

Figure 17: Mapping of the elements which entered strain softening stage in the plane strain specimen using the non-local model with $l_{\mathrm{c}}=50[\mu \mathrm{m}]$ and the introduction of a crack following Thomason coalescence criterion (medium refined mesh) (a) at the failure initiation, (b) at a point during the failure process and (c) near the total failure.

respectively in 16598 and 16116 6-node triangular elements of $75[\mu \mathrm{m}]$ size with an aspect ratio around to 7 and 5. Moreover, the DG-stability parameter is increased (here the value $\beta_{s}=175$ is considered) because of the use of different elements (axisymmetric and not 2D ones). Besides, the term $\frac{1}{2} \frac{\partial \llbracket \boldsymbol{u} \rrbracket}{\partial \boldsymbol{X}}$ in Eq. (61) has been neglected. The material model and parameters are identical, as defined in the Section 5.2.1 and $l_{\mathrm{c}}=50[\mu \mathrm{m}]$ is considered.

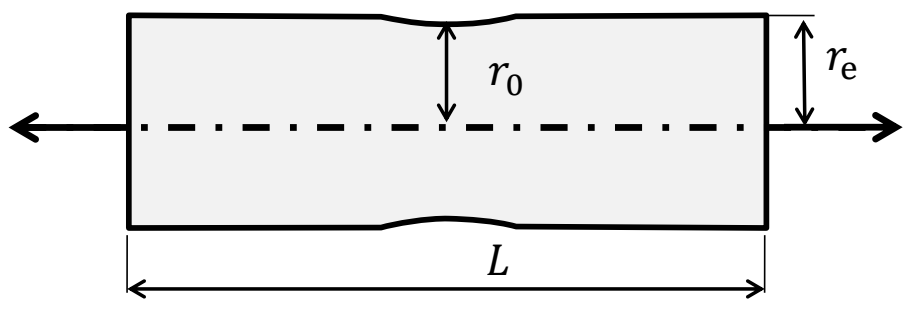

(a)

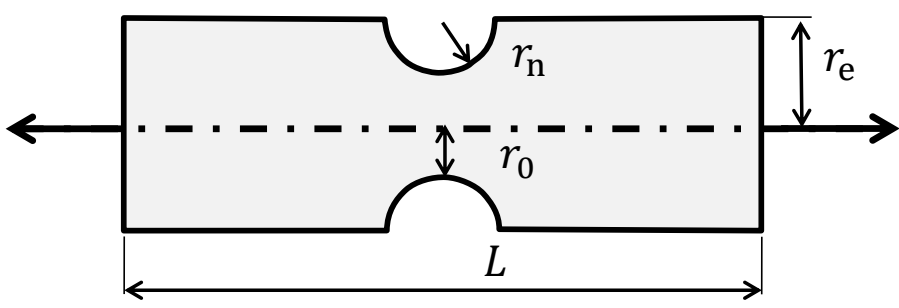

(b)

Figure 18: Geometries (a) of the round bar and (b) of the notched bar.

The variation of the tensile force applied on the smooth round bar is represented in Fig. 19 in terms of the radius reduction for $l_{\mathrm{c}}=50[\mu \mathrm{m}]$. A good correlation is obtained with both results presented by both Besson et al. (2001) and Huespe et al. (2012). Besides, the force evolution is also shown in Fig. 19 for different values of $k_{\omega}$. As announced in Section 5.2.1, one can notice that $k_{\omega}$ has no influence on the crack initiation as the Lode term is vanishing near the bar center. However, once the crack is propagating, the stress state changes and 


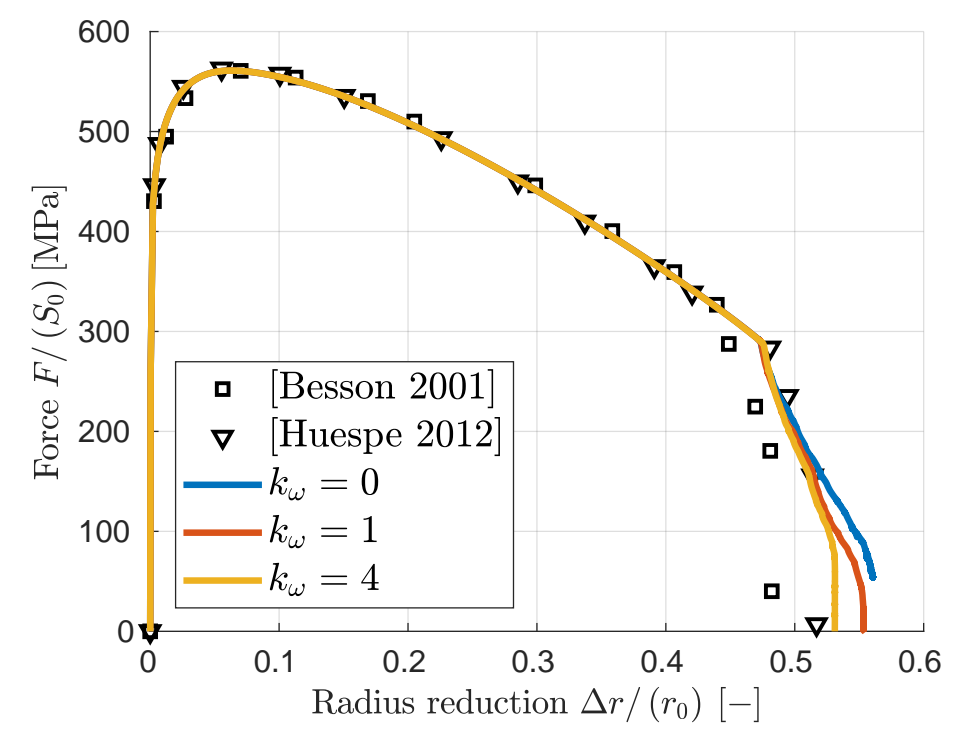

Figure 19: Variation of the applied force as a function of the central radius reduction of the smooth round bar for the non-local Gurson-Thomason model with $l_{\mathrm{c}}=50[\mu \mathrm{m}]$ and the CBM, and comparison with the results of Besson et al. (2001) and Huespe et al. (2012) (respectively square and triangle markers).

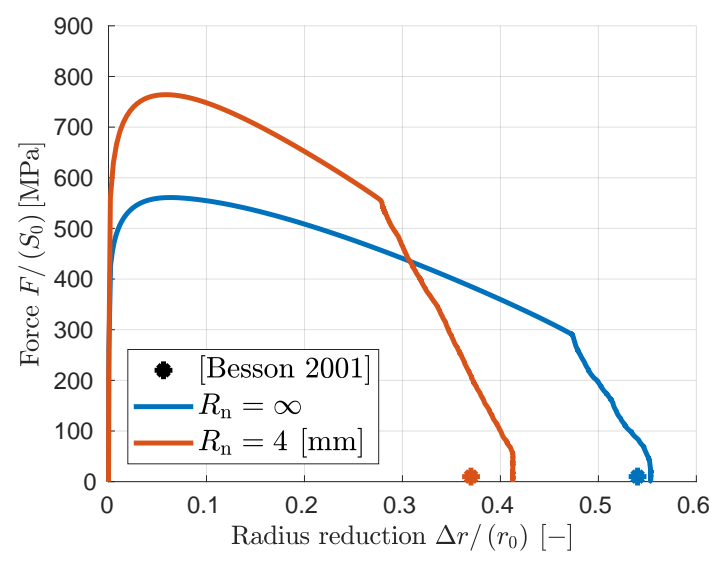

(a) $l_{\mathrm{c}}=50[\mu \mathrm{m}]$

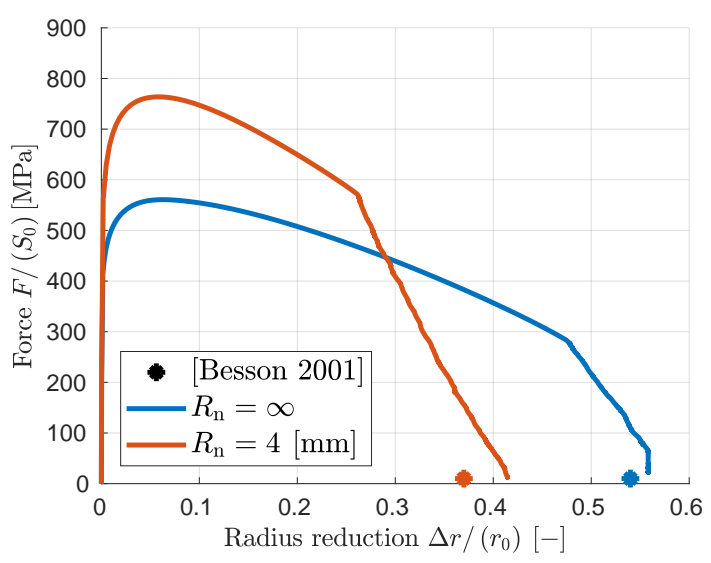

(b) $l_{\mathrm{c}}=75[\mu \mathrm{m}]$

Figure 20: Variation of the applied force as a function of the minimum cross-section radius reduction of the smooth and notched round bars for the non-local Gurson-Thomason model with the CBM for two values of $l_{\mathrm{c}}$, and comparison with the experimental failure strain given by Besson et al. (2001) (star markers).

the $k_{\omega}$ effect becomes noticeable. The force evolutions for the two notch radii are compared in Fig. 20(a). A good correlation is obtained between the failure strain measured by Besson et al. (2001) despite the fact that the numerical results slightly overestimate the real failure strain.

Figs. 21(a) to 21(c) represent the deformed state of the smooth round bar at different points of the process as the plastic strain distribution for $k_{\omega}=1$. At a necking factor of 0.5 , 
a tensile crack initiates at the center. It radially propagates while shear bands are starting to develop aside of the crack (see Fig. 21(b)). At some point, the crack changes its direction, following those shear bands until it reaches the surface. A cup-cone failure is thus obtained as experimentally observed by Besson et al. (2001).

The plastic strain distributions in the deformed state of the notched bar are presented in Figs. 21(d) to 21(f). The crack also starts from the center of the specimen but at a different plastic strain value. Indeed, the increase of the stress triaxiality, caused by a smaller notch radius, leads to an earlier failure initiation. At some point, the crack also bifurcates by following a shear band, resulting in a cup-cone failure mode as observed experimentally by Besson et al. (2001).

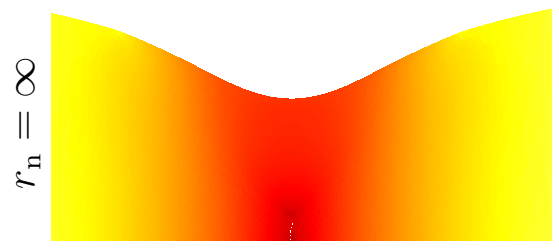

(a)

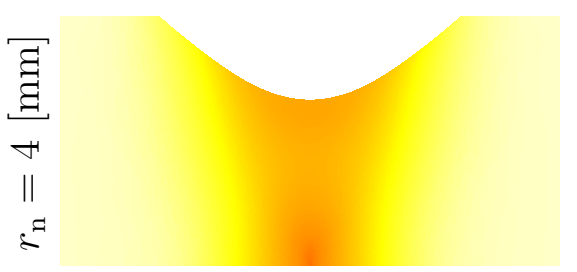

(d)

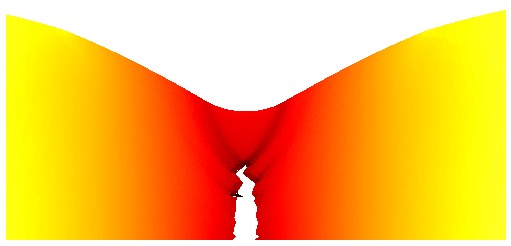

(b)

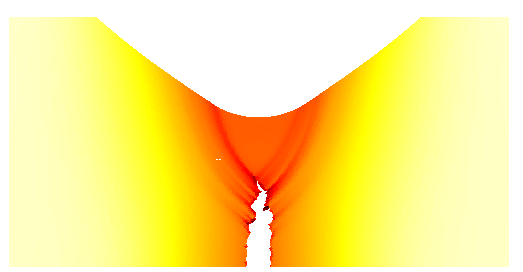

(e)

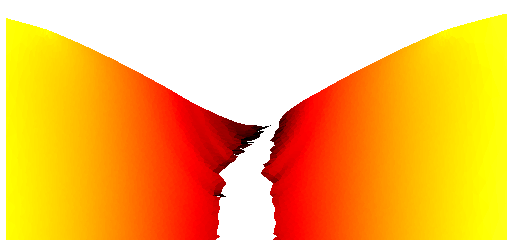

(c)

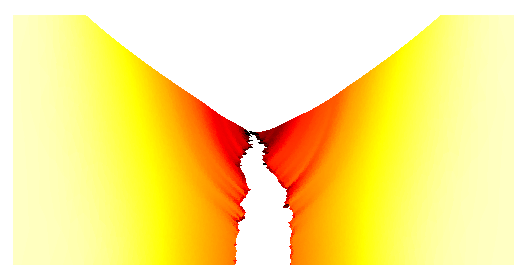

(f)

$\hat{p} \quad 0.75 \quad>1.5$

Figure 21: Matrix plastic strain distribution of the smooth $\left(r_{\mathrm{n}}=\infty\right)$ and the notched $\left(r_{\mathrm{n}}=4[\mathrm{~mm}]\right)$ round bar specimen using the non-local model with $l_{\mathrm{c}}=50[\mu \mathrm{m}]$ and introducing a crack following Thomason coalescence criterion: (a) at failure initiation, (b) before crack tilting, and (c) at total failure.

\subsubsection{Influence of the non-local length}

Results presented in Sections 5.2.2 and 5.2.3 are now compared with the results obtained with the second set of material parameters calibrated in Section 5.2 .1 for $l_{\mathrm{c}}=75[\mu \mathrm{m}]$. Similar results are obtained, either in terms of force evolution or fracture surface. As an illustration, the force evolution in terms of, respectively, the thickness and the diameter reductions are compared in Figs. 15(b) and 20(b), showing that the model can still predict the correct trends and similar conclusions can be drawn about the results. The model is effectively rich enough to represent the slant and cup-cone fracture, characteristic of the ductile failure process. However, the simple procedure based on the information available to calibrate the model is unable to determine a unique set of material parameters. As several parameters combinations can reproduce the expected behaviour, a more complete strategy 
needs to be adopted, based on physical arguments involving for instance the determination of the tearing resistance and fracture energy in problems with stable crack propagation.

\section{Conclusions}

We have developed a damage to crack transition numerical framework in order to predict the failure of porous ductile materials. This framework couples an implicit non-local damage model, which captures the initial diffuse damage stage, with a cohesive band model, which captures the void coalescence and crack extension opening stage. The cohesive band model allows considering complex 3D stress state effects during the final failure process. The transition has been numerically implemented within a discontinuous Galerkin formulation.

The Gurson-Tvergaard-Needleman (GTN) model was selected to simulate the void growth before the coalescence phase. First, in order to compare our results with the literature, a local model was considered and the cracks were inserted in a local model at the loss of ellipticity. Then, in order to illustrate the versatility of the non-local framework, we have considered the Thomason coalescence criterion as crack initiation indicator, and the Thomason coalescence model as crack opening governing law. In particular, it was shown that the Thomason coalescence criterion can be met during the strain softening stage, motivating the use of a non-local damage formulation.

The framework was then applied to reproduce the slant and cup-cone failure modes of plane-strain and axisymmetric specimens, respectively. In particular, it was shown that the numerical predictions converge with the mesh refinement, and that the cohesive band thickness governs the fracture energy released during the coalescence stage, and possibly the failure mode when vanishing values are considered.

The material model could be enhanced with more complex coalescence models: in this paper, slant and cup-cone failures are captured because of the creation of the free surface. Nguyen et al. (Submitted) have recently introduced a non-local multi-surface approach accounting for void growth, internal necking and shearing mechanisms capturing these patterns without introducing a discontinuity. This non-local porous plasticity model will improve the quality of the predictions at low stress triaxiality.

Another step should be focus on the material parameters calibration. A proper procedure should be developed, followed by a suitable experimental campaign. In particular, crack propagation tests (for instance, compact tension specimen) could help to determine the values of the characteristic lengths involved in this model.

\section{Acknowledgement}

The research has been funded by the Walloon Region under the agreement no.7581MRIPF in the context of the 16th MECATECH call.

\section{Appendix A. Formulation of the plastic corrector}

The details on the determination of the plastic corrector omitted in Section 2.4 are presented here. 


\section{Appendix A.1. Plastic flow and corrector stress}

In case of plastic flow, the first step is to re-expressed the plastic correction $\Delta \gamma \mathbf{N}^{\mathrm{p}}$ and the stress $\boldsymbol{\tau}$ in terms of the predictor state and of the unknown plastic increments. If plastic flow occurs, the plastic deformation gradient is updated following Eq. (44). The corresponding corrected elastic strain tensors are

$$
\begin{aligned}
& \mathbf{F}^{\mathrm{e}}=\mathbf{F} \cdot \mathbf{F}_{\mathrm{pr}}^{\mathrm{p}-1} \cdot\left[\exp \left(\Delta \gamma \mathbf{N}^{\mathrm{p}}\right)\right]^{-1}, \text { and } \\
& \mathbf{E}^{\mathrm{e}}=\mathbf{E}_{\mathrm{pr}}^{\mathrm{e}}-\Delta \gamma \mathbf{N}^{\mathrm{p}} .
\end{aligned}
$$

From these relations, the corotational Kirchhoff stress components are computed thanks to Eqs. (21) and (43), using the flow decomposition (45),

$$
\begin{cases}\boldsymbol{\tau}^{\mathrm{dev}} & =\left(\boldsymbol{\tau}_{\mathrm{pr}}\right)^{\mathrm{dev}}-3 G \Delta \hat{d} \frac{\boldsymbol{\tau}^{\mathrm{dev}}}{\tau^{\mathrm{eq}}} \\ p^{\prime} & =p_{\mathrm{pr}}^{\prime}-K \Delta \hat{q}\end{cases}
$$

This relation directly gives the relationship between the corrected pressure $p^{\prime}$ in terms of the predictor stress and the volumetric plastic increment $\Delta \hat{q}$. The deviatoric counterpart is obtained by exploiting the chosen form of the decomposition (45). Indeed, the predictor and corrected deviatoric stresses are coaxial as shown by rewriting Eq. (A.2) as

$$
\left[\frac{\tau^{\mathrm{eq}}+3 G \Delta \hat{d}}{\tau^{\mathrm{eq}}}\right] \boldsymbol{\tau}^{\mathrm{dev}}=\left(\boldsymbol{\tau}_{\mathrm{pr}}\right)^{\mathrm{dev}}
$$

One has also, from the definition of the equivalent stress for both tensors,

$$
\left(\tau^{\mathrm{eq}}\right)^{2}=\frac{3}{2}(\boldsymbol{\tau})^{\mathrm{dev}}:(\boldsymbol{\tau})^{\mathrm{dev}}=\frac{3}{2}\left[\frac{\tau^{\mathrm{eq}}}{\tau^{\mathrm{eq}}+3 G \Delta \hat{d}}\right]^{2}\left(\boldsymbol{\tau}_{\mathrm{pr}}\right)^{\mathrm{dev}}:\left(\boldsymbol{\tau}_{\mathrm{pr}}\right)^{\mathrm{dev}}=\left[\frac{\tau^{\mathrm{eq}}}{\tau^{\mathrm{eq}}+3 G \Delta \hat{d}}\right]^{2}\left(\tau_{\mathrm{pr}}^{\mathrm{eq}}\right)^{2}
$$

which finally gives, after some manipulations, the sought relation

$$
\tau^{\mathrm{eq}}=\tau_{\mathrm{pr}}^{\mathrm{eq}}-3 G \Delta \hat{d} \text { and } \tau^{\mathrm{dev}}=\frac{\tau_{\mathrm{pr}}^{\mathrm{eq}}-3 G \Delta \hat{d}}{\tau_{\mathrm{pr}}^{\mathrm{eq}}}\left(\boldsymbol{\tau}_{\mathrm{pr}}\right)^{\mathrm{dev}}
$$

Furthermore, the deviatoric plastic correction can be simplified by this last equation:

$$
\Delta \gamma \mathbf{N}^{\mathrm{pdev}}=\Delta \hat{d} \frac{3 \boldsymbol{\tau}^{\mathrm{dev}}}{2 \tau^{\mathrm{eq}}}=\Delta \hat{d} \frac{3\left(\boldsymbol{\tau}_{\mathrm{pr}}\right)^{\mathrm{dev}}}{2 \tau_{\mathrm{pr}}^{\mathrm{eq}}}
$$

which shows the advantage of this formulation in which the deviatoric part of the normal is constant during the plastic correction. 


\section{Appendix A.2. Residual form}

Now, based on previous results, the residual form $\boldsymbol{r}_{\mathrm{p}}(\boldsymbol{v})$ from Eq. (47) is developed. The first equation $r_{\mathrm{p}_{1}}$ corresponds to the consistency condition associated with the yield surface after plastic correction:

$$
r_{\mathrm{p} 1}=\phi\left(\tau^{\mathrm{eq}}, p^{\prime}, \tau_{\mathrm{Y}}, \breve{Z}\right)
$$

involving $\tau^{\mathrm{eq}}=\tau_{\mathrm{pr}}^{\mathrm{eq}}-3 G \Delta \hat{d}$ and $p^{\prime}=p_{\mathrm{pr}}^{\prime}-K \Delta \hat{q}$. The second equation links the volumetric and the deviatoric plastic increments through the normality rule. From Eqs. (24) and (45), one has

$$
\Delta \gamma=\frac{\Delta \hat{d}}{N^{\mathrm{d}}}=\frac{\Delta \hat{q}}{N^{\mathrm{q}}}
$$

or again,

$$
r_{\mathrm{p} 2}=\Delta \hat{d} N^{\mathrm{q}}-\Delta \hat{q} N^{\mathrm{d}}
$$

The third equation results from the integration of Eq. (29),

$$
\left(1-f_{\mathrm{V}_{0}}\right) \tau_{\mathrm{Y}} \Delta \hat{p}=\boldsymbol{\tau}: \Delta \gamma \mathbf{N}^{\mathrm{p}}
$$

Using again Eq. (45), one has

$$
r_{\mathrm{p} 3}=\left(1-f_{\mathrm{V}_{0}}\right) \tau_{\mathrm{Y}} \Delta \hat{p}-\tau^{\mathrm{eq}} \Delta \hat{d}-p^{\prime} \Delta \hat{q}
$$

To this set of equations is added the internal variables evolution laws as constraints. For given values of the set $\boldsymbol{v}$, the hardening law $\tau_{\mathrm{Y}}=\tau_{\mathrm{Y}}\left(\hat{p}^{n}+\Delta \hat{p}\right)$ gives the expression of the yield stress in terms of the matrix plastic increment. The porosity growth equation (26) is rewritten by including the different growth term expressions and the flow description (24) as

$$
\dot{f_{\mathrm{V}}}=A_{\mathrm{n}}(\hat{p}) \dot{\hat{p}}+\left(1-f_{\mathrm{V}}\right) \dot{\hat{q}}+B_{\mathrm{n}} f_{\mathrm{V}} \dot{\hat{d}} .
$$

After using backward Euler integration, the porosity increment is obtained by

$$
\Delta f_{\mathrm{V}}=\frac{\left(1-f_{\mathrm{V}}^{n}\right) \Delta \hat{q}+A_{\mathrm{n}}(\hat{p}) \Delta \hat{p}+B_{\mathrm{n}}\left(\zeta_{\mathrm{pr}}\right) f_{\mathrm{V}}^{n} \Delta \hat{d}}{1+\Delta \hat{q}-B_{\mathrm{n}}\left(\zeta_{\mathrm{pr}}\right) \Delta \hat{d}}
$$

In Eq. (A.13), the Lode variable at the predictor $\zeta_{\mathrm{pr}}$ is used as the plastic flow leaves it unchanged. Then, the current ligament ratio (31) is computed using

$$
\chi=\chi^{n}\left(\frac{\breve{f}_{\mathrm{V}}}{\breve{f}_{\mathrm{V}}^{n}}\right)^{\frac{1}{3}} \exp \left(\frac{1}{3} \kappa \Delta \hat{p}\right)
$$

and the current cell aspect ratio given by

$$
\lambda=\lambda^{n} \exp (\kappa \Delta \hat{p}) .
$$


Exponential regulation is applied to smoothly avoid out-of range values on the porosity and the ligament ratio. Equations are also all dimensionless.

The problem is solved using a Newton-Raphson scheme from an initial guess $\boldsymbol{v}^{(0)}$ and the subsequent solutions $\boldsymbol{v}^{(i+1)}=\boldsymbol{v}^{(i)}+\Delta \boldsymbol{v}$ obtained with

$$
\mathbf{J}_{\boldsymbol{v}}\left(\boldsymbol{v}^{(i)}\right) \Delta \boldsymbol{v}=-\boldsymbol{r}_{\mathrm{p}}\left(\boldsymbol{v}^{(i)}\right)
$$

introducing $\mathbf{J}_{\boldsymbol{v}}=\frac{\partial \boldsymbol{r}_{\mathrm{p}}}{\partial \boldsymbol{v}}$, the Jacobian matrix. Its components are

$$
\begin{aligned}
& J_{v_{1, \Delta \hat{d}}}=-3 G N^{\mathrm{d}}, \\
& J_{v 1, \Delta \hat{q}}=-K N^{\mathrm{q}} \text {, } \\
& J_{v 1, \Delta \hat{p}}=\frac{\partial \phi}{\partial \tau_{\mathrm{Y}}} h+\frac{\partial \phi}{\partial \chi} \frac{\partial \chi}{\partial \Delta \hat{p}}, \\
& J_{v_{2, \Delta \hat{d}}}=N^{\mathrm{q}}-3 \Delta \hat{d} \frac{\partial N^{\mathrm{q}}}{\partial \tau^{\mathrm{eq}}} G+3 \Delta \hat{q} \frac{\partial N^{\mathrm{d}}}{\partial \tau^{\mathrm{eq}}} G, \\
& J_{v 2, \Delta \hat{q}}=-\Delta \hat{d} \frac{\partial N^{\mathrm{q}}}{\partial p^{\prime}} K-N^{\mathrm{d}}+\Delta \hat{q} \frac{\partial N^{\mathrm{d}}}{\partial p^{\prime}} K, \\
& J_{v 2, \Delta \hat{p}}=\Delta \hat{d} \frac{\partial N^{\mathrm{q}}}{\partial \tau_{\mathrm{Y}}} h-\Delta \hat{q} \frac{\partial N^{\mathrm{d}}}{\partial \tau_{\mathrm{Y}}} h, \\
& J_{v_{3, \Delta \hat{d}}}=-\tau^{\mathrm{eq}}+3 G \Delta \hat{d}, \\
& J_{v 3, \Delta \hat{q}}=-p^{\prime}+K \Delta \hat{q}, \\
& J_{v 3, \Delta \hat{p}}=\left(1-f_{\mathrm{V}_{0}}\right)\left(\tau_{\mathrm{Y}}+h \Delta \hat{p}\right),
\end{aligned}
$$

where $h$ corresponds to $\frac{\partial \tau_{\mathrm{Y}}}{\partial \hat{p}}$. One has to specify $\phi, N^{\mathrm{d}}, N^{\mathrm{q}}$, and their derivatives with respect to $\tau^{\mathrm{eq}}, p^{\prime}, \tau_{\mathrm{Y}}$ and other internal variables for the involved strain mechanisms in order to complete the procedure.

\section{Appendix A.2.1. Gurson residual form}

When the Gurson model is used, the residues involve the yield surface (32) and its derivatives. The derivatives of $\phi_{\mathrm{G}}$ are given by

$$
N_{\mathrm{G}}^{\mathrm{d}}=2 \frac{\tau^{\mathrm{eq}}}{\tau_{\mathrm{Y}}^{2}} \text { and } N_{\mathrm{G}}^{\mathrm{q}}=\frac{3 q_{1} q_{2} \breve{f}_{\mathrm{V}}}{\tau_{\mathrm{Y}}} \sinh \left(\frac{3 q_{2} p^{\prime}}{2 \tau_{\mathrm{Y}}}\right)
$$

and

$$
\frac{\partial \phi_{\mathrm{G}}}{\partial \tau_{\mathrm{Y}}}=-2 \frac{\tau^{\mathrm{eq} 2}}{\tau_{\mathrm{Y}}^{3}}-\frac{3 q_{1} q_{2} p^{\prime} \breve{f}_{\mathrm{V}}}{\tau_{\mathrm{Y}}^{2}} \sinh \left(\frac{3 q_{2} p^{\prime}}{2 \tau_{\mathrm{Y}}}\right) \text { and } \frac{\partial \phi_{\mathrm{G}}}{\partial \chi}=0
$$

while the derivatives of the normal are

$$
\begin{gathered}
\frac{\partial N_{\mathrm{G}}^{\mathrm{d}}}{\partial \tau^{\mathrm{eq}}}=\frac{2}{\tau_{\mathrm{Y}}^{2}} \text { and } \frac{\partial N_{\mathrm{G}}^{\mathrm{q}}}{\partial \tau^{\mathrm{eq}}}=0, \\
\frac{\partial N_{\mathrm{G}}^{\mathrm{d}}}{\partial p^{\prime}}=0 \text { and } \frac{\partial N_{\mathrm{G}}^{\mathrm{q}}}{\partial p^{\prime}}=\frac{9 q_{1} q_{2}^{2} \breve{f}_{\mathrm{V}}}{2 \tau_{\mathrm{Y}}^{2}} \cosh \left(\frac{3 q_{2} p^{\prime}}{2 \tau_{\mathrm{Y}}}\right),
\end{gathered}
$$

and

$$
\frac{\partial N_{\mathrm{G}}^{\mathrm{d}}}{\partial \tau_{\mathrm{Y}}}=-4 \frac{\tau^{\mathrm{eq}}}{\tau_{\mathrm{Y}}^{3}} \text { and } \frac{\partial N_{\mathrm{G}}^{\mathrm{q}}}{\partial \tau_{\mathrm{Y}}}=-\frac{9 q_{1} q_{2}^{2} \breve{f}_{\mathrm{V}} p^{\prime}}{2 \tau_{\mathrm{Y}}^{3}} \cosh \left(\frac{3 q_{2} p^{\prime}}{2 \tau_{\mathrm{Y}}}\right)-\frac{3 q_{1} q_{2} \breve{f}_{\mathrm{V}}}{\tau_{\mathrm{Y}}^{2}} \sinh \left(\frac{3 q_{2} p^{\prime}}{2 \tau_{\mathrm{Y}}}\right) .
$$




\section{Appendix A.2.2. Thomason residual form}

When Thomason model is used, the yield surface (38) steps in the residues $\boldsymbol{r}_{\mathrm{p}}(\boldsymbol{v})$. The derivatives of $\phi_{\mathrm{T}}$ are

$$
\frac{\partial \phi_{\mathrm{T}}}{\partial \tau_{\mathrm{Y}}}=-C_{\mathrm{T}}^{\phi} \text { and } \frac{\partial \phi_{\mathrm{T}}}{\partial \chi}=-\tau_{\mathrm{Y}} \frac{\partial C_{\mathrm{T}}^{\phi}}{\partial \chi}
$$

with

$$
\begin{aligned}
\frac{\partial C_{\mathrm{T}}^{\phi}}{\partial \chi}=-(2 \chi) & {\left[\alpha(1 / \chi-1)^{2}+\beta \sqrt{1 / \chi}\right] } \\
- & \left(1-\chi^{2}\right)\left[2 \alpha(1 / \chi-1)\left(1 / \chi^{2}\right)+\frac{1}{2} \beta \chi^{-3 / 2}\right] .
\end{aligned}
$$

The normal components are

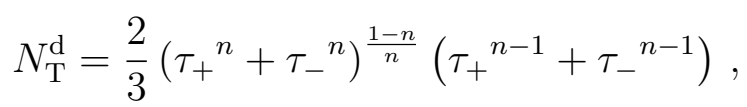

and

$$
N_{\mathrm{T}}^{\mathrm{q}}=\left(\tau_{+}{ }^{n}+\tau_{-}{ }^{n}\right)^{\frac{1-n}{n}}\left(\tau_{+}{ }^{n-1}-\tau_{-}{ }^{n-1}\right) .
$$

The derivatives of the normal components are

$$
\begin{aligned}
& \frac{\partial N_{\mathrm{T}}^{\mathrm{d}}}{\partial \tau^{\mathrm{eq}}}=\frac{4}{9}(n-1)\left[\left({\tau_{+}}^{n}+\tau_{-}{ }^{n}\right)^{\frac{1}{n}-1}\left({\tau_{+}}^{n-2}+\tau_{-}{ }^{n-2}\right)\right. \\
& \left.-\left(\tau_{+}{ }^{n}+\tau_{-}{ }^{n}\right)^{\frac{1}{n}-2}\left(\tau_{+}{ }^{n-1}+\tau_{-}{ }^{n-1}\right)^{2}\right], \\
& \frac{\partial N_{\mathrm{T}}^{\mathrm{q}}}{\partial \tau^{\mathrm{eq}}}=\frac{2}{3}(n-1)\left[\left({\tau_{+}}^{n}+\tau_{+}{ }^{n}\right)^{\frac{1}{n}-1}\left(\tau_{-}{ }^{n-2}-\tau_{-}{ }^{n-2}\right)\right. \\
& \left.-\left(\tau_{+}{ }^{n}+\tau_{-}{ }^{n}\right)^{\frac{1}{n}-2}\left(\tau_{+}{ }^{2 n-2}-{\tau_{-}}^{2 n-2}\right)\right], \\
& \frac{\partial N_{\mathrm{T}}^{\mathrm{d}}}{\partial p^{\prime}}=\frac{2}{3}(n-1)\left[\left({\tau_{+}}^{n}+\tau_{-}{ }^{n}\right)^{\frac{1}{n}-1}\left(\tau_{+}{ }^{n-2}-\tau_{-}{ }^{n-2}\right)\right. \\
& \left.-\left(\tau_{+}{ }^{n}+\tau_{-}{ }^{n}\right)^{\frac{1}{n}-2}\left(\tau_{+}{ }^{2 n-2}-{\tau_{-}}^{2 n-2}\right)\right], \\
& \frac{\partial N_{\mathrm{T}}^{\mathrm{q}}}{\partial p^{\prime}}=(n-1)\left[\left(\tau_{+}{ }^{n}+\tau_{-}{ }^{n}\right)^{\frac{1}{n}-1}\left(\tau_{+}{ }^{n-2}+\tau_{-}{ }^{n-2}\right)\right. \\
& \left.-\left(\tau_{+}{ }^{n}+\tau_{-}{ }^{n}\right)^{\frac{1}{n}-2}\left({\tau_{+}}^{n-1}-\tau_{-}{ }^{n-1}\right)^{2}\right], \\
& \frac{\partial N^{\mathrm{d}}}{\partial \tau_{\mathrm{Y}}}=0 \quad \text { and } \quad \frac{\partial N^{\mathrm{q}}}{\partial \tau_{\mathrm{Y}}}=0 .
\end{aligned}
$$




\section{Appendix B. Formulation of the material operators}

The material operator is obtained by linearising the output of the bulk constitutive law in terms of the input. Four terms are therefore required: $\frac{\partial \mathbf{P}}{\partial \mathbf{F}}, \frac{\partial f_{\mathrm{V}}}{\partial \mathbf{F}}, \frac{\partial \mathbf{P}}{\partial \tilde{f}_{\mathrm{V}}}, \frac{\partial f_{\mathrm{V}}}{\partial \tilde{f}_{\mathrm{V}}}$.

\section{Appendix B.1. Stress tensor derivatives}

From its definition (48), the derivative of the first Piola-Kirchhoff tensor reads

$$
\begin{aligned}
\frac{\partial \mathbf{P}}{\partial \mathbf{F}}= & \frac{\partial \mathbf{F}^{\mathrm{e}}}{\partial \mathbf{F}} \cdot\left(\boldsymbol{\tau}: \mathcal{L}^{\mathrm{e}}\right) \cdot \mathbf{F}^{\mathrm{p}-T}+\mathbf{F}^{\mathrm{e}} \cdot 3\left(\frac{\partial \boldsymbol{\tau}}{\partial \mathbf{F}}{ }^{1,2}: \mathcal{L}^{\mathrm{e}}\right){ }^{4} \cdot \mathbf{F}^{\mathrm{p}-T} \\
& +\mathbf{F}^{\mathrm{e}} \cdot\left(\boldsymbol{\tau}: \frac{\partial \mathcal{L}^{\mathrm{e}}}{\partial \mathbf{F}}\right) \cdot \mathbf{F}^{\mathrm{p}-T}+\mathbf{F}^{\mathrm{e}} \cdot\left(\boldsymbol{\tau}: \mathcal{L}^{\mathrm{e}}\right) \cdot \frac{\partial \mathbf{F}^{\mathrm{p}-T}}{\partial \mathbf{F}}, \\
\frac{\partial \mathbf{P}}{\partial \tilde{f}_{\mathrm{V}}}= & \frac{\partial \mathbf{F}^{\mathrm{e}}}{\partial \tilde{f}_{\mathrm{V}}} \cdot\left(\mathcal{L}^{\mathrm{e}}: \boldsymbol{\tau}\right) \cdot \mathbf{F}^{\mathrm{p}-\mathrm{T}}+\mathbf{F}^{\mathrm{e}} \cdot\left(\frac{\partial \boldsymbol{\tau}}{\partial \tilde{f}_{\mathrm{V}}}: \mathcal{L}^{\mathrm{e}}\right) \cdot \mathbf{F}^{\mathrm{p}-T} \\
& +\mathbf{F}^{\mathrm{e}} \cdot\left(\boldsymbol{\tau}: \frac{\partial \mathcal{L}^{\mathrm{e}}}{\partial \tilde{f}_{\mathrm{V}}}\right) \cdot \mathbf{F}^{\mathrm{p}-T}+\mathbf{F}^{\mathrm{e}} \cdot\left(\boldsymbol{\tau}: \mathcal{L}^{\mathrm{e}}\right) \cdot \frac{\partial \mathbf{F}^{\mathrm{p}-T}}{\partial \tilde{f}_{\mathrm{V}}},
\end{aligned}
$$

where $\left[\mathbf{A} \cdot{ }^{3} \mathcal{B}\right]_{i j k l}=A_{k m} \mathcal{B}_{i j m l}$.

In Eqs. (B.1) and (B.2), the derivative of each factor has still to be developed. For this purpose, one has for:

- The inverse of the plastic deformation mapping $\mathbf{F}^{\mathrm{p}-1}$ :

$$
\left\{\begin{array}{l}
\frac{\partial F^{\mathrm{p}}-1}{\partial F_{k L}}=-F_{I M}^{\mathrm{p}-1} \frac{\partial F_{M N}^{\mathrm{p}}}{\partial F_{k L}} F_{N J}^{\mathrm{p}-1} \\
\frac{\partial F^{\mathrm{p}}-1}{\partial \tilde{f}_{\mathrm{V}}}=-F_{I M}^{\mathrm{p}}-1 \frac{\partial F_{M N}^{\mathrm{p}}}{\partial \tilde{f}_{\mathrm{V}}} F_{N J}^{\mathrm{p}}-1
\end{array}\right.
$$

- The elastic deformation gradient $\mathbf{F}^{\mathrm{e}}$ :

$$
\left\{\begin{array}{l}
\frac{\partial F_{i J}^{\mathrm{e}}}{\partial F_{k L}}=\delta_{i k} F^{\mathrm{p}-1}+F_{i M} \frac{\partial F_{M J}^{\mathrm{p}-1}}{\partial F_{k L}} \\
\frac{\partial F_{i J}^{\mathrm{e}}}{\partial \tilde{f}_{\mathrm{V}}}=F_{i M} \frac{\partial F^{\mathrm{p}}-1}{\partial \tilde{f}_{\mathrm{V}}}
\end{array}\right.
$$

- The approximation of the strain measure derivatives $\mathcal{L}^{\mathrm{e}}$ :

$$
\frac{\partial \mathcal{L}^{\mathrm{e}}}{\partial \mathbf{F}}=\frac{\partial \mathcal{L}^{\mathrm{e}}}{\partial \mathbf{C}^{\mathrm{e}}}: \frac{\partial \mathbf{C}^{\mathrm{e}}}{\partial \mathbf{F}^{\mathrm{e}}}: \frac{\partial \mathbf{F}^{\mathrm{e}}}{\partial \mathbf{F}} \quad \text { and } \quad \frac{\partial \mathcal{L}^{\mathrm{e}}}{\partial \tilde{f}_{\mathrm{V}}}=\frac{\partial \mathcal{L}^{\mathrm{e}}}{\partial \mathbf{C}^{\mathrm{e}}}: \frac{\partial \mathbf{C}^{\mathrm{e}}}{\partial \mathbf{F}^{\mathrm{e}}}: \frac{\partial \mathbf{F}^{\mathrm{e}}}{\partial \tilde{f}_{\mathrm{V}}} .
$$

Combining Eqs. (B.3), (B.4) and (B.5), the derivatives (B.1) - (B.2) can be expressed in terms of the derivatives of $\mathbf{F}^{\mathrm{p}}$ and $\boldsymbol{\tau}$ only. The derivative with respect to $\mathbf{F}$ in the previous equations can be substituted by a derivative with respect to $\mathbf{E}_{\mathrm{pr}}^{\mathrm{e}}$ by using the chain rule and the following relation

$$
\frac{\partial E_{\mathrm{pr} I J}^{\mathrm{e}}}{\partial F_{k L}}=\frac{1}{2} \mathcal{L}_{\mathrm{pr}_{I J M N}}^{\mathrm{e}}\left(F_{\mathrm{pr}_{k N}}^{\mathrm{e}} F_{\mathrm{pr} L M}^{\mathrm{p}-1}+F_{\mathrm{pr}_{k M}}^{\mathrm{e}} F_{\mathrm{pr} L N}^{\mathrm{p}-1}\right),
$$


obtained from the Eqs. (41), (42) and the definition of $\mathcal{L}_{\mathrm{pr}}^{\mathrm{e}}=\frac{\partial \ln \mathbf{C}_{\mathrm{pr}}^{\mathrm{e}}}{\partial \mathbf{C}_{\mathrm{pr}}^{\mathrm{e}}}$.

In the case of an elastic step, no plastic flow occurs and it follows that the only non-trivial derivative is

$$
\frac{\partial \boldsymbol{\tau}}{\partial \mathbf{E}_{\mathrm{pr}}^{\mathrm{e}}}=2 G \mathcal{I}^{\mathrm{dev}}+K \mathbf{I} \otimes \mathbf{I}
$$

obtained from Eq. (21), with $\mathcal{I}^{\text {dev }}=\mathcal{I}-\frac{1}{3} \mathbf{I} \otimes \mathbf{I}$ and $\mathcal{I}$ the fourth-order symmetric unit tensor.

Otherwise, in case of plasticity, the derivatives of the plastic deformation mapping are obtained from its definition (44), leading to

$$
\begin{aligned}
& \frac{\partial \mathbf{F}^{\mathrm{p} T}}{\partial \mathbf{E}_{\mathrm{pr}}^{\mathrm{e}}}=\mathbf{F}_{\mathrm{pr}}^{\mathrm{p} \mathrm{T}} \cdot \frac{\partial \exp \left(\Delta \gamma \mathbf{N}^{\mathrm{p}}\right)}{\partial \mathbf{E}_{\mathrm{pr}}^{\mathrm{e}}}=\mathbf{F}_{\mathrm{pr}}^{\mathrm{p} \mathrm{T}} \cdot \mathcal{E}: \frac{\partial\left(\Delta \gamma \mathbf{N}^{\mathrm{p}}\right)}{\partial \mathbf{E}_{\mathrm{pr}}^{\mathrm{e}}}, \\
& \frac{\partial \mathbf{F}^{\mathrm{p} T}}{\partial \tilde{f}_{\mathrm{V}}}=\mathbf{F}_{\mathrm{pr}}^{\mathrm{p} T} \cdot \frac{\partial \exp \left(\Delta \gamma \mathbf{N}^{\mathrm{p}}\right)}{\partial \tilde{f}_{\mathrm{V}}}=\mathbf{F}_{\mathrm{pr}}^{\mathrm{p}} \cdot \mathcal{E}: \frac{\partial\left(\Delta \gamma \mathbf{N}^{\mathrm{p}}\right)}{\partial \tilde{\mathrm{V}}_{\mathrm{V}}},
\end{aligned}
$$

where $\mathcal{E}=\frac{\partial \exp \left(\Delta \gamma \mathbf{N}^{\mathrm{p}}\right)}{\partial \Delta \gamma \mathbf{N}^{\mathrm{p}}}$ depends on the approximation used to compute the tensor exponential. The plastic corrections appearing here above, computed by Eq. (45) and (A.6), are also derived

$$
\begin{aligned}
\frac{\partial \Delta \gamma \mathbf{N}^{\mathrm{p}}}{\partial \mathbf{E}_{\mathrm{pr}}^{\mathrm{e}}} & =\frac{3\left(\boldsymbol{\tau}_{\mathrm{pr}}\right)^{\text {dev }}}{2 \tau_{\mathrm{pr}}^{\mathrm{eq}}} \otimes \frac{\partial \Delta \hat{d}}{\partial \mathbf{E}_{\mathrm{pr}}^{\mathrm{e}}}+\frac{3 G \Delta \hat{d}}{\tau_{\mathrm{pr}}^{\mathrm{eq}}}\left(\mathcal{I}^{\mathrm{dev}}-\frac{3\left(\boldsymbol{\tau}_{\mathrm{pr}}\right)^{\operatorname{dev}} \otimes\left(\boldsymbol{\tau}_{\mathrm{pr}}\right)^{\operatorname{dev}}}{\tau_{\mathrm{pr}}^{\mathrm{eq}}}\right)+\frac{1}{3} \mathbf{I} \otimes \frac{\partial \Delta \hat{q}}{\partial \mathbf{E}_{\mathrm{pr}}^{\mathrm{e}}}, \\
\frac{\partial \Delta \gamma \mathbf{N}^{\mathrm{p}}}{\partial \tilde{f}_{\mathrm{V}}} & =\frac{\partial \Delta \hat{d}}{\partial \tilde{f}_{\mathrm{V}}} \frac{3\left(\boldsymbol{\tau}_{\mathrm{pr}}\right)^{\mathrm{dev}}}{2 \tau_{\mathrm{pr}}^{\mathrm{eq}}}+\frac{1}{3} \frac{\partial \Delta \hat{q}}{\partial \tilde{f}_{\mathrm{V}}} \mathbf{I} .
\end{aligned}
$$

The Kirchhoff stress is also linearised from Eqs. (A.2)-(A.5) and one finally has

$$
\begin{aligned}
\frac{\partial \boldsymbol{\tau}}{\partial \mathbf{E}_{\mathrm{pr}}^{\mathrm{er}}=} & \left(1-\frac{3 G \Delta \hat{d}}{\tau_{\mathrm{pr}}^{\mathrm{eq}}}\right) 2 G \mathcal{I}^{\mathrm{dev}}-\frac{3 G}{\tau_{\mathrm{pr}}^{\mathrm{eq}}}\left(\boldsymbol{\tau}_{\mathrm{pr}}\right)^{\operatorname{dev}} \otimes\left[\frac{\partial \Delta \hat{d}}{\partial \mathbf{E}_{\mathrm{pr}}^{\mathrm{e}}}-\frac{3 G \Delta \hat{d}\left(\boldsymbol{\tau}_{\mathrm{pr}}\right)^{\operatorname{dev}}}{2 \tau_{\mathrm{pr}}^{\mathrm{eq}} 2}\right] \\
& +K \mathbf{I} \otimes\left(\mathbf{I}-\frac{\partial \Delta \hat{q}}{\partial \mathbf{E}_{\mathrm{pr}}^{\mathrm{e}}}\right) \\
\frac{\partial \boldsymbol{\tau}}{\partial \tilde{f}_{\mathrm{V}}}= & -\frac{3 G}{\tau_{\mathrm{pr}}^{\mathrm{eq}}} \frac{\partial \Delta \hat{d}}{\partial \hat{f}_{\mathrm{V}}}\left(\boldsymbol{\tau}_{\mathrm{pr}}\right)^{\operatorname{dev}}-K \frac{\partial \Delta \hat{q}}{\partial \hat{f}_{\mathrm{V}}} \mathbf{I} .
\end{aligned}
$$

The last missing steps are the derivatives of the plastic increments, computed through Eq. (50) and the results of Appendix B.3.

\section{Appendix B.2. Porosity derivatives}

The derivatives of the local porosity is obtained by derivation of Eq. (A.13)

$$
\begin{aligned}
& \frac{\partial f_{\mathrm{V}}}{\partial \mathbf{E}_{\mathrm{pr}}^{\mathrm{e}}}=\frac{\partial f_{\mathrm{V}}}{\partial B_{\mathrm{n}}} \frac{\partial B_{\mathrm{n}}}{\partial \mathbf{E}_{\mathrm{pr}}^{\mathrm{e}}}+\frac{\partial f_{\mathrm{V}}}{\partial \Delta \hat{d}} \frac{\partial \Delta \hat{d}}{\partial \mathbf{E}_{\mathrm{pr}}^{\mathrm{e}}}+\frac{\partial f_{\mathrm{V}}}{\partial \Delta \hat{q}} \frac{\partial \Delta \hat{q}}{\partial \mathbf{E}_{\mathrm{pr}}^{e}}+\frac{\partial f_{\mathrm{V}}}{\partial \Delta \hat{p}} \frac{\partial \Delta \hat{p}}{\partial \mathbf{E}_{\mathrm{pr}}^{\mathrm{e}}}, \\
& \frac{\partial f_{\mathrm{V}}}{\partial \tilde{f}_{\mathrm{V}}}=\frac{\partial f_{\mathrm{V}}}{\partial \Delta \hat{d}} \frac{\partial \Delta \hat{d}}{\partial \tilde{\mathrm{f}}_{\mathrm{V}}}+\frac{\partial f_{\mathrm{V}}}{\partial \Delta \hat{q}} \frac{\partial \Delta \hat{q}}{\partial \tilde{f}_{\mathrm{V}}}+\frac{\partial f_{\mathrm{V}}}{\partial \Delta \hat{p}} \frac{\partial \Delta \hat{p}}{\partial \tilde{f}_{\mathrm{V}}}
\end{aligned}
$$

combined with Eq. (B.6) to obtain the derivatives in terms of the deformation gradient. In addition, one has

$$
\frac{\partial B_{\mathrm{n}}}{\partial \mathbf{E}_{\mathrm{pr}}^{\mathrm{e}}}=-4 G k_{\omega} \zeta_{\mathrm{pr}}^{2}\left[\left(\boldsymbol{\tau}_{\mathrm{pr}}\right)^{\mathrm{dev}-\mathrm{T}}-\frac{9}{2} \frac{\left(\boldsymbol{\tau}_{\mathrm{pr}}\right)^{\mathrm{dev}}}{\tau_{\mathrm{pr}}^{\mathrm{eq}}}\right]: \mathcal{I}^{\mathrm{dev}}
$$


and

$$
\begin{aligned}
\frac{\partial f_{\mathrm{V}}}{\partial \Delta \hat{d}} & =\frac{B_{\mathrm{n}}}{1+\Delta \hat{q}-B_{\mathrm{n}} \Delta \hat{d}} f_{\mathrm{V}} \\
\frac{\partial f_{\mathrm{V}}}{\partial \Delta \hat{q}} & =\frac{1}{1+\Delta \hat{q}-B_{\mathrm{n}} \Delta \hat{d}}\left(1-f_{\mathrm{V}}\right) \\
\frac{\partial f_{\mathrm{V}}}{\partial \Delta \hat{p}} & =\frac{A_{\mathrm{n}}+\frac{\partial A_{\mathrm{n}}}{\partial \hat{p}} \Delta \hat{p}}{1+\Delta \hat{q}-B_{\mathrm{n}} \Delta \hat{d}}
\end{aligned}
$$

Appendix B.3. Internal variables derivatives

In order to complete the tangent operator formulation, the derivatives of the plastic increment in terms of the predictor strain, appearing in Eq. (49), are obtained thanks to

$$
\frac{\partial r_{\mathrm{p}_{i}}}{\partial \mathbf{E}_{\mathrm{pr}}^{\mathrm{e}}}=\frac{\partial r_{\mathrm{p}_{i}}}{\partial \tau_{\mathrm{pr}}^{\mathrm{eq}}} \frac{\partial \tau_{\mathrm{pr}}^{\mathrm{eq}}}{\partial \mathbf{E}_{\mathrm{pr}}^{\mathrm{e}}}+\frac{\partial r_{\mathrm{p}_{i}}}{\partial p_{\mathrm{pr}}^{\prime}} \frac{\partial p_{\mathrm{pr}}^{\prime}}{\partial \mathbf{E}_{\mathrm{pr}}^{\mathrm{e}}}=\frac{\partial r_{\mathrm{p}_{i}}}{\partial \tau_{\mathrm{pr}}^{\mathrm{eq}}} \frac{3 G\left(\boldsymbol{\tau}_{\mathrm{pr}}\right)^{\mathrm{dev}}}{\tau_{\mathrm{pr}}^{\mathrm{eq}}}+\frac{\partial r_{\mathrm{p}_{i}}}{\partial p_{\mathrm{pr}}^{\prime}} K \mathbf{I},
$$

with

$$
\begin{array}{ll}
\frac{\partial r_{\mathrm{p} 1}}{\partial \tau_{\mathrm{pr}}^{\mathrm{eq}}}=N^{\mathrm{d}}, & \frac{\partial r_{\mathrm{p} 2}}{\partial \tau_{\mathrm{pr}}^{\mathrm{eq}}}=\Delta \hat{d} \frac{\partial N^{\mathrm{q}}}{\partial \tau^{\mathrm{eq}}}-\Delta \hat{q} \frac{\partial N^{\mathrm{d}}}{\partial \tau^{\mathrm{eq}}}, \quad \frac{\partial r_{\mathrm{p} 3}}{\partial \tau_{\mathrm{pr}}^{\mathrm{eq}}}=-\Delta \hat{d}, \text { and } \\
\frac{\partial r_{\mathrm{p} 1}}{\partial p_{\mathrm{pr}}^{\prime}}=N^{\mathrm{q}}, & \frac{\partial r_{\mathrm{p} 2}}{\partial p_{\mathrm{pr}}^{\prime}}=\Delta \hat{d} \frac{\partial N^{\mathrm{q}}}{\partial p^{\prime}}-\Delta \hat{q} \frac{\partial N^{\mathrm{d}}}{\partial p^{\prime}}, \quad \frac{\partial r_{\mathrm{p} 3}}{\partial p_{\mathrm{pr}}^{\prime}}=-\Delta \hat{q} .
\end{array}
$$

The derivatives with respect to the non-local porosity are given by:

$$
\frac{\partial r_{\mathrm{p} 1}}{\partial \tilde{f}_{\mathrm{V}}}=\frac{\partial \phi}{\partial \breve{f}_{\mathrm{V}}}, \quad \frac{\partial r_{\mathrm{p} 2}}{\partial \tilde{f}_{\mathrm{V}}}=\Delta \hat{d} \frac{\partial N^{\mathrm{q}}}{\partial \breve{f}_{\mathrm{V}}}-\Delta \hat{q} \frac{\partial N^{\mathrm{d}}}{\partial \breve{f}_{\mathrm{V}}} \quad \text { and } \quad \frac{\partial r_{\mathrm{p} 3}}{\partial \tilde{f}_{\mathrm{V}}}=0
$$

where the derivatives of $\phi$ and $\mathrm{N}^{\mathrm{p}}$ depend on the considered strain mechanisms.

\section{Appendix B.3.1. Gurson model}

If the Gurson model is used, the derivatives of the yield surface are

$$
\frac{\partial \phi_{\mathrm{G}}}{\partial \breve{f}_{\mathrm{V}}}=2 q_{1} \cosh \left(\frac{3 q_{2} p^{\prime}}{2 \tau_{\mathrm{Y}}}\right)-2 q_{3}^{2} \breve{f}_{\mathrm{V}}
$$

while those of the plastic normal read

$$
\frac{\partial N_{\mathrm{G}}^{\mathrm{d}}}{\partial \breve{f}_{\mathrm{V}}}=0 \quad \text { and } \quad \frac{\partial N_{\mathrm{G}}^{\mathrm{q}}}{\partial \breve{f}_{\mathrm{V}}}=\frac{3 q_{1} q_{2}}{\tau_{\mathrm{Y}}} \sinh \left(\frac{3 q_{2} p^{\prime}}{2 \tau_{\mathrm{Y}}}\right)
$$

Appendix B.3.2. Thomason model

The derivatives of $\phi$ and $\mathbf{N}^{\mathrm{p}}$ in terms of the effective porosity are

$$
\frac{\partial \phi_{\mathrm{T}}}{\partial \breve{f}_{\mathrm{V}}}=-\tau_{\mathrm{Y}} \frac{\partial C_{\mathrm{T}}^{\phi}}{\partial \chi} \frac{\partial \chi}{\partial \breve{f}_{\mathrm{V}}}
$$

and

$$
\frac{\partial N_{\mathrm{G}}^{\mathrm{d}}}{\partial \breve{f}_{\mathrm{V}}}=0 \quad \text { and } \quad \frac{\partial N_{\mathrm{G}}^{\mathrm{q}}}{\partial \breve{f}_{\mathrm{V}}}=0
$$




\section{Appendix C. Formulation in the local form}

This section presents the modifications required to bring the constitutive law under its local form.

\section{Appendix C.1. Local residual form}

The local residual form (51) is computed in a similar way as the non-local one (47). However, the effective porosity is no longer fixed at the predictor state by the non-local increment and Eq. (A.13) is no longer aside but is coupled within the Newton-Raphson scheme. The Jacobian matrix (A.16) of the system is consequently modified:

$$
\begin{gathered}
J_{v_{1, \Delta \hat{d}}}=-3 G N^{\mathrm{d}}+\left(\frac{\partial \phi}{\partial \breve{f}_{\mathrm{V}}}+\frac{\partial \phi}{\partial \chi} \frac{\partial \chi}{\partial \breve{f}_{\mathrm{V}}}\right) \frac{\partial f_{\mathrm{V}}}{\partial \Delta \hat{d}}, \\
J_{v 1, \Delta \hat{q}}=-K N^{\mathrm{q}}+\left(\frac{\partial \phi}{\partial \breve{f}_{\mathrm{V}}}+\frac{\partial \phi}{\partial \chi} \frac{\partial \chi}{\partial \breve{f}_{\mathrm{V}}}\right) \frac{\partial f_{\mathrm{V}}}{\partial \Delta \hat{q}}, \\
J_{v 1, \Delta \hat{p}}=\frac{\partial \phi}{\partial \tau_{\mathrm{Y}}} h+\left(\frac{\partial \phi}{\partial \check{f}_{\mathrm{V}}}+\frac{\partial \phi}{\partial \chi} \frac{\partial \chi}{\partial \breve{f}_{\mathrm{V}}}\right) \frac{\partial f_{\mathrm{V}}}{\partial \Delta \hat{p}}+\frac{\partial \phi}{\partial \chi} \frac{\partial \chi}{\partial \Delta \hat{p}}, \\
J_{v_{2, \Delta \hat{d}}=} N^{\mathrm{q}}+\Delta \hat{d}\left(\frac{\partial N^{\mathrm{q}}}{\partial \breve{f}_{\mathrm{V}}} \frac{\partial f_{\mathrm{V}}}{\partial \Delta \hat{d}}-3 G \frac{\partial N^{\mathrm{q}}}{\partial \tau^{\mathrm{eq}}}\right)-\Delta \hat{q}\left(\frac{\partial N^{\mathrm{d}}}{\partial \breve{f}_{\mathrm{V}}} \frac{\partial f_{\mathrm{V}}}{\partial \Delta \hat{d}}-3 G \frac{\partial N^{\mathrm{d}}}{\partial \tau^{\mathrm{eq}}}\right), \\
J_{v 2, \Delta \hat{q}}=\Delta \hat{d}\left(\frac{\partial N^{\mathrm{q}}}{\partial \breve{f}_{\mathrm{V}}} \frac{\partial f_{\mathrm{V}}}{\partial \Delta \hat{q}}-K \frac{\partial N^{\mathrm{q}}}{\partial p^{\prime}}\right)-N^{\mathrm{d}}-\Delta \hat{q}\left(\frac{\partial N^{\mathrm{d}}}{\partial \breve{f}_{\mathrm{V}}} \frac{\partial f_{\mathrm{V}}}{\partial \Delta \hat{q}}-K \frac{\partial N^{\mathrm{d}}}{\partial p^{\prime}}\right), \\
J_{v 2, \Delta \hat{p}}=\Delta \hat{d}\left(\frac{\partial N^{\mathrm{q}}}{\partial \breve{f}_{\mathrm{V}}} \frac{\partial f_{\mathrm{V}}}{\partial \Delta \hat{p}}+\frac{\partial N^{\mathrm{q}}}{\partial \tau_{\mathrm{Y}}} h\right)-\Delta \hat{q}\left(\frac{\partial N^{\mathrm{d}}}{\partial \breve{f}_{\mathrm{V}}} \frac{\partial f_{\mathrm{V}}}{\partial \Delta \hat{p}}+\frac{\partial N^{\mathrm{d}}}{\partial \tau_{\mathrm{Y}}} h\right),
\end{gathered}
$$

and $J_{v 3, \boldsymbol{v}}$ is left unchanged. The derivatives appearing in the previous expressions can be found in Appendix A and Appendix B.

\section{Appendix C.2. Formulation of the local material operators}

The procedure is similar to the method explained in Section 2.4.3 and Appendix B. Equations (B.1) to (B.10) are still applicable to express $\frac{\partial \mathbf{P}}{\partial \mathbf{F}}$ in terms of the plastic increment $\boldsymbol{v}$. These derivatives are extracted using Eq. (50) and the corresponding modified version of Eq. (B.14) for the residues derivatives reads

$$
\frac{\partial r_{\mathrm{p}_{i}}}{\partial \mathbf{E}_{\mathrm{pr}}^{\mathrm{e}}}=\frac{\partial r_{\mathrm{p}_{i}}}{\partial \tau_{\mathrm{pr}}^{\mathrm{eq}}} \frac{3 G\left(\boldsymbol{\tau}_{\mathrm{pr}}\right)^{\text {dev }}}{\tau_{\mathrm{pr}}^{\mathrm{eq}}}+\frac{\partial r_{\mathrm{p}_{i}}}{\partial p_{\mathrm{pr}}^{\prime}} K \mathbf{I}+\frac{\partial r_{\mathrm{p}_{i}}}{\partial \breve{f}_{\mathrm{V}}} \frac{\partial f_{\mathrm{V}}}{\partial \mathbf{E}_{\mathrm{pr}}^{\mathrm{e}}} .
$$

\section{Appendix D. Formulation of the finite element forces}

The expressions of the finite element forces appearing in Eq. (75) are presented here. The weak form (72)-(73) developed in Section 4.1.2 is the starting point of the finite element discretisation. By taking into account the discretisation (74) and the discontinuities between elements, one has the following elementary terms:

- Inertial forces $\mathbf{M}^{e a b} \cdot \ddot{\boldsymbol{u}}^{b}$ with:

$$
M_{i j}^{e a b}=\int_{B^{e}} \rho_{0} N^{a} N^{b} \delta_{i j} d V_{0}
$$


- Internal forces $\boldsymbol{f}_{u \text { int }}^{e}{ }^{a}$ :

$$
f_{u \text { int } i}^{e}=\int_{B^{e}} P_{i K}\left(\nabla_{0} N^{a}\right)_{K} d V_{0}
$$

- External forces $\boldsymbol{f}_{u \text { ext }}^{e}$ :

$$
f_{u \operatorname{ext} i}^{e}=\int_{B^{e}} \rho_{0} b_{0 i} N^{a} d V_{0}+\int_{S_{\mathrm{N}}^{s}} \bar{t}_{0 i} N^{a} d S_{0} ;
$$

- Interface forces $\boldsymbol{f}_{u \mathrm{I}}^{s} a^{ \pm}$:

$$
\begin{aligned}
f_{u \mathrm{I} i}^{s} a^{ \pm}= & \int_{S_{\mathrm{IC}}^{s}}\left( \pm N^{a^{ \pm}}\right)\left\langle P_{\mathrm{b} i K}\right\rangle N_{\mathrm{I} K}^{-} d S_{0}+\int_{S_{\mathrm{IU}}^{s}}\left( \pm N^{a^{ \pm}}\right)\left\langle P_{i K}\right\rangle N_{\mathrm{I} K}^{-} d S_{0} \\
& +\int_{S_{\mathrm{IU}}^{s}}\left( \pm N^{a^{ \pm}}\right) N_{\mathrm{I} K}^{-}\left\langle\frac{\beta_{s}}{h_{s}} \mathcal{C}_{i K m N}^{\mathrm{e}}\right\rangle \llbracket u_{m} \rrbracket N_{\mathrm{I}_{N}}^{-} d S_{0} \\
& +\frac{1}{2} \int_{S_{\mathrm{IU}}^{s}} \llbracket u_{k} \rrbracket \mathcal{C}^{\mathrm{e}}{ }_{k M i N}^{ \pm}\left(\nabla_{0} N^{a^{ \pm}}\right)_{N} N_{\mathrm{I}_{M}^{-}}^{-} d S_{0} .
\end{aligned}
$$

- Internal non-local forces $f_{\tilde{Z} \text { int }}^{e}{ }^{a}$ :

$$
f_{\tilde{Z} \text { int }}^{e}{ }^{a}=\int_{B^{e}}\left(\Delta \tilde{Z} N^{a}+\left(\nabla_{0} N^{a}\right)_{K} C_{1 K L}\left(\nabla_{0} \Delta \tilde{Z}\right)_{L}\right) d V_{0} ;
$$

- Internal local forces $f_{Z \text { int }}^{e}{ }^{a}$ :

$$
f_{Z \text { int }}^{e}=\int_{B^{e}} \Delta Z N^{a} d V_{0} ;
$$

- Interface non-local forces $f_{\tilde{Z} \mathrm{I}}^{s} a^{a^{ \pm}}$:

$$
\begin{aligned}
f_{\tilde{Z} \mathrm{I}}^{s a^{ \pm}}= & \int_{S_{\mathrm{IU}}^{s}}\left( \pm N^{a^{ \pm}}\right)\left\langle C_{1 K L}\left(\nabla_{0} \Delta \tilde{Z}\right)_{L}\right\rangle N_{\mathrm{I}_{K}}^{-} d S_{0} \\
& +\int_{S_{\mathrm{IU}}^{s}}\left( \pm N^{a^{ \pm}}\right) N_{\mathrm{I} K}^{-}\left\langle\frac{\beta_{s}}{h_{s}} C_{1 K L}\right\rangle \llbracket \Delta \tilde{Z} \rrbracket N_{\mathrm{I}_{L}}^{-} d S_{0} \\
& +\frac{1}{2} \int_{S_{\mathrm{IU}}^{s}} \llbracket \Delta \tilde{Z} \rrbracket N_{\mathrm{I}_{K}^{-}} C_{\mathrm{I}_{K L}}^{ \pm}\left(\nabla_{0} N^{a^{ \pm}}\right)_{L} d S_{0} .
\end{aligned}
$$

In those expressions, the superscripts $a, b, \ldots$ stand for the node $a$ which belongs to a volume element $B^{e}$ or to a interface one $S^{s}$. The sign + or - in the case of interface elements differentiates if the node (i.e. $a^{+}$or $a^{-}$) belongs to $B^{e^{+}}$or $B^{e^{-}}$. Practically, only the degrees of freedom are duplicated at the interfaces and not the node itself. The computation of the volume element force involves the shape functions of the volume elements. All nodes of both volume elements are therefore contributing in the interface term thanks to the symmetrisation term. Only the gradient of the jump displacement uses interface shape functions to naturally respect condition (62). The quadratic volume elements are integrated using a reduced integration while the surface element are fully-integrated to avoid spurious penetration modes. 


\section{Appendix E. Formulation of the finite element stiffness matrices}

The expressions of the contributions to finite element stiffness matrix $\mathbf{K}$ appearing in Eq. (80) are detailed here. The stiffness matrix regroups all the stiffness components resulting from the linearisation of the elementary forces in equations (D.1) to (D.7). One obtains

$$
\mathbf{K}=\left[\begin{array}{cc}
\mathbf{K}_{\boldsymbol{u} u \text { int }}+\mathbf{K}_{\boldsymbol{u} \boldsymbol{u} \mathrm{I}} & \mathbf{K}_{\boldsymbol{u} \tilde{Z} \text { int }}+\mathbf{K}_{\boldsymbol{u} \tilde{Z} \mathrm{I}} \\
\mathbf{K}_{\tilde{Z} \boldsymbol{u} \text { int }}-\mathbf{K}_{Z \boldsymbol{u} \text { int }}+\mathbf{K}_{\tilde{Z} \boldsymbol{u} \mathrm{I}} & \mathbf{K}_{\tilde{Z} \tilde{Z} \text { int }}-\mathbf{K}_{Z \tilde{Z} \text { int }}+\mathbf{K}_{\tilde{Z} \tilde{Z} \mathrm{I}}
\end{array}\right],
$$

with

- Internal force stiffness terms $\mathbf{K}_{\boldsymbol{u} \boldsymbol{u} \text { int }}^{e}{ }^{a b}$ and $\mathbf{K}_{\boldsymbol{u} \tilde{Z} \text { int }}^{e}{ }^{a b}$ :

$$
\begin{gathered}
K_{\boldsymbol{u} \boldsymbol{u} \text { int } i j}^{e}=\frac{\partial f_{\boldsymbol{u} \text { int } i}^{e}}{\partial u_{j}^{b}}=\int_{B^{e}} \mathcal{C}_{\boldsymbol{u} \mathbf{F} i K j L}\left(\nabla_{0} N^{a}\right)_{K}\left(\nabla_{0} N^{b}\right)_{L} d V_{0} ; \\
K_{\boldsymbol{u} \tilde{Z} \text { int } i}^{e b}=\frac{\partial f_{\boldsymbol{u} \text { int } i}^{e}}{\partial \tilde{Z}^{b}}=\int_{B^{e}} C_{\boldsymbol{u} \tilde{Z}_{i K}}\left(\nabla_{0} N^{a}\right)_{K} N^{b} d V_{0} ;
\end{gathered}
$$

- Interface force stiffness terms $\mathbf{K}_{\boldsymbol{u} \boldsymbol{u} \mathrm{I}}^{s} a^{ \pm} b^{ \pm}$and $\mathbf{K}_{\boldsymbol{u} \tilde{Z} \mathrm{I}}^{s} a^{a^{ \pm} b^{ \pm}}$:

$$
\begin{aligned}
& K_{\boldsymbol{u} \boldsymbol{u} i j}^{s a^{ \pm} b^{ \pm}}=\frac{\partial f_{\boldsymbol{u} \mathrm{I} i}^{s} a^{ \pm}}{\partial u_{j}^{b^{ \pm}}}=+\frac{1}{2} \int_{S_{\mathrm{IC}}^{s}}\left( \pm N^{a^{ \pm}}\right) \mathcal{C}_{\boldsymbol{u} \mathbf{F} i K j L}^{\mathrm{b} \stackrel{ \pm}{ \pm}}\left(\nabla_{0} N^{b^{ \pm}}\right)_{L} N_{\mathrm{I} K}^{-} d S_{0} \\
& +\int_{S_{\mathrm{IC}}^{s}}\left( \pm N^{a^{ \pm}}\right)\left\langle\mathfrak{C}_{\boldsymbol{u} \llbracket \boldsymbol{u} \rrbracket_{i K j}}^{\mathrm{b}}\right\rangle\left( \pm N^{b^{ \pm}}\right) N_{\mathrm{I} K}^{-} d S_{0} \\
& +\int_{S_{\mathrm{IC}}^{s}}^{\mathrm{C}}\left( \pm N^{a^{ \pm}}\right)\left\langle\mathcal{C}_{u \nabla \llbracket \boldsymbol{b} \rrbracket_{i K j L}}^{\mathrm{b}}\right\rangle\left( \pm \nabla_{0} N^{b^{ \pm}}\right)_{L} N_{\mathrm{I} K}^{-} d S_{0} \\
& +\frac{1}{2} \int_{S_{\mathrm{IU}}^{s}}\left( \pm N^{a^{ \pm}}\right) \mathcal{C}_{u \mathbf{F}_{i K j L}}^{ \pm}\left(\nabla_{0} N^{b^{ \pm}}\right)_{L} N_{\mathrm{I} K}^{-} d S_{0} \\
& +\int_{S_{\mathrm{IU}}^{S}}\left( \pm N^{a \pm}\right) N_{\mathrm{I} K}^{-}\left\langle\frac{\beta_{s}}{h_{s}} \mathcal{C}_{i K j N}^{\mathrm{e}}\right\rangle\left( \pm N^{b^{ \pm}}\right) N_{\mathrm{I}}^{-} d S_{0} \\
& +\frac{1}{2} \int_{S_{\mathrm{IU}}^{s}}\left( \pm N^{b \pm}\right) \mathcal{C}_{j M i N}^{\mathrm{e}}{ }^{ \pm}\left(\nabla_{0} N^{a \pm}\right)_{N} N_{\mathrm{I}_{M}}^{-} d S_{0} ; \\
& K_{\boldsymbol{u} \tilde{Z} \mathrm{I} i j}^{a^{ \pm} b^{ \pm}}=\frac{\partial f_{\boldsymbol{u} i}^{s} a_{i}^{ \pm}}{\partial \tilde{Z}^{b^{ \pm}}}=\frac{1}{2} \int_{S_{\mathrm{IU}}^{s}}\left( \pm N^{a}\right) C_{\boldsymbol{u} \tilde{Z}_{i K}^{ \pm}}^{ \pm} N^{b^{ \pm}} N_{\mathrm{I} K}^{-}
\end{aligned}
$$

- Internal non-local force stiffness terms $\boldsymbol{K}_{\tilde{Z} \boldsymbol{u} \text { int }}^{e}{ }^{a b}$ and $K_{\tilde{Z} \tilde{Z} \text { int }}^{e}{ }^{a b}$ :

$$
\begin{gathered}
K_{\tilde{Z} \boldsymbol{u} \text { int } j}^{e}=\frac{\partial f_{\tilde{Z} \text { int }}^{e}}{\partial u_{j}^{b}}=0 ; \\
K_{\tilde{Z} \tilde{Z} \text { int }}^{e}{ }^{a b}=\frac{\partial f_{\tilde{Z} \text { int }}^{e}{ }^{a}}{\partial \tilde{Z}^{b}}=\int_{B^{e}}\left[N^{a} N^{b}+\left(\nabla_{0} N^{a}\right)_{K} C_{l K L}\left(\nabla_{0} N^{b}\right)_{L}\right] d V_{0} ;
\end{gathered}
$$


- Internal local force stiffness terms $\boldsymbol{K}_{Z \boldsymbol{u} \text { int }}^{e}{ }^{a b}$ and $K_{Z \tilde{Z} \text { int }}^{e}{ }^{a b}$ :

$$
\begin{gathered}
K_{Z \boldsymbol{u} \text { int } j}^{e}{ }^{a b}=\frac{\partial f_{Z \text { int }}^{e}{ }^{a}}{\partial u_{j}^{b}}=\int_{B^{e}} C_{Z \mathbf{F} j K} N^{a}\left(\nabla_{0} N^{b}\right)_{K} d V_{0} \\
K_{Z \tilde{Z} \text { int }}^{e}{ }^{a b}=\frac{\partial f_{Z \text { int }}^{e}{ }^{a}}{\partial \tilde{Z}^{b}}=\int_{B^{e}} C_{Z \tilde{Z}^{2}} N^{a} N^{b} d V_{0}
\end{gathered}
$$

- Interface non-local force stiffness terms $\boldsymbol{K}_{\tilde{Z} \boldsymbol{u} \mathrm{I}}^{s}{ }^{a^{ \pm} b^{ \pm}}$and $K_{\tilde{Z} \tilde{Z} \mathrm{I}}^{s}{ }^{a^{ \pm} b^{ \pm}}$:

$$
\begin{aligned}
& K_{\tilde{Z} u \mathrm{I} j}^{s} \underset{a^{ \pm} b^{ \pm}}{S}=0 \\
& K_{\tilde{Z} \tilde{Z} \mathrm{I}}^{a^{ \pm} b^{ \pm}}=\frac{\partial f_{\tilde{Z} \mathrm{I}}^{s{ }^{a \pm}}}{\partial \tilde{Z}^{b^{ \pm}}}=\frac{1}{2} \int_{S_{\mathrm{IU}}^{s}}\left( \pm N^{a \pm}\right) C_{1 K L}\left(\nabla_{0} N^{b^{ \pm}}\right)_{L} N_{\mathrm{I} K}^{-} d S_{0} \\
& +\int_{S_{\mathrm{IU}}^{s}}^{\mathrm{U}}\left( \pm N^{a \pm}\right) N_{\mathrm{I} K}^{-}\left\langle\frac{\beta_{s}}{h_{s}} C_{\mathrm{l} K L}\right\rangle\left( \pm N^{b^{ \pm}}\right) N_{\mathrm{I}}^{-}{ }_{L} d S_{0} \\
& +\frac{1}{2} \int_{S_{\mathrm{IU}}^{s}}\left( \pm N^{b^{ \pm}}\right) C_{\mathrm{l}_{K} L}^{ \pm}\left(\nabla_{0} N^{a^{ \pm}}\right)_{L} N_{\mathrm{I} K}^{-} d S_{0} \text {. }
\end{aligned}
$$

The expressions above involve material tangent tensors computed in Appendix B. If the considered point is not located on a cohesive band, the relations are directly obtained

$$
\mathcal{C}_{\boldsymbol{u} \mathbf{F}}=\frac{\partial \mathbf{P}}{\partial \mathbf{F}}, \mathbf{C}_{\boldsymbol{u} \tilde{Z}}=\frac{\partial \mathbf{P}}{\partial \Delta \tilde{f}_{\mathrm{V}}}, \mathbf{C}_{Z \mathbf{F}}=\frac{\partial \Delta f_{\mathrm{V}}}{\partial \mathbf{F}}, C_{Z \tilde{Z}}=\frac{\partial \Delta f_{\mathrm{V}}}{\partial \Delta \tilde{f}_{\mathrm{V}}} .
$$

If the point is located on a cohesive band model, the derivatives of the band deformation gradient $\mathbf{F}_{\mathrm{b}}$ have to be included. They directly derived from the computation of $\mathbf{F}_{\mathrm{b}}(60)$

$$
\delta F_{\mathrm{b}_{i J}}^{ \pm}=\delta F_{i J}^{ \pm}+\frac{N_{\mathrm{IJ}}}{h_{\mathrm{b}}} \delta \llbracket u_{i} \rrbracket+\frac{1}{2} \delta\left(\nabla_{0} \llbracket u_{i} \rrbracket\right)_{J} .
$$

From the previous equation, the tangent stiffness terms are thus:

$$
\begin{gathered}
\mathcal{C}_{\boldsymbol{u} \mathbf{F}}^{\mathrm{b}}=\frac{\partial \mathbf{P}_{\mathrm{b}}}{\partial \mathbf{F}_{\mathrm{b}}}, \\
\mathfrak{C}_{\boldsymbol{u} \llbracket \boldsymbol{u} \rrbracket}^{\mathrm{b}}=\frac{\partial \mathbf{P}_{\mathrm{b}}}{\partial \mathbf{F}_{\mathrm{b}}} \cdot \frac{\boldsymbol{N}_{\mathrm{I}}}{h_{\mathrm{b}}}, \\
\mathcal{C}_{\boldsymbol{u} \boldsymbol{\nabla} \llbracket \boldsymbol{u} \rrbracket}^{\mathrm{b}}=\frac{1}{2} \frac{\partial \mathbf{P}_{\mathrm{b}}}{\partial \mathbf{F}_{\mathrm{b}}} .
\end{gathered}
$$

where the derivative of $\mathbf{P}_{\mathrm{b}}$ is computing using the local model. 
Aduloju, S.C., Truster, T.J., 2019. A variational multiscale discontinuous galerkin formulation for both implicit and explicit dynamic modeling of interfacial fracture. Computer Methods in Applied Mechanics and Engineering 343, 602 - 630. URL: http://www.sciencedirect.com/science/article/pii/ S0045782518304201, doi:https://doi.org/10.1016/j.cma.2018.08.025.

Aldakheel, F., 2017. Micromorphic approach for gradient-extended thermo-elasticplastic solids in the logarithmic strain space. Continuum Mechanics and Thermodynamics 29, 1207-1217. URL: http: //link.springer.com/10.1007/s00161-017-0571-0, doi:10.1007/s00161-017-0571-0.

Aldakheel, F., Wriggers, P., Miehe, C., 2018. A modified gurson-type plasticity model at finite strains: formulation, numerical analysis and phase-field coupling. Computational Mechanics 62, 815-833. URL: https://doi.org/10.1007/s00466-017-1530-0, doi:10.1007/s00466-017-1530-0.

Barenblatt, G., 1962. The mathematical theory of equilibrium cracks in brittle fracture, Elsevier. volume 7 of Advances in Applied Mechanics, pp. 55 - 129. URL: http://www.sciencedirect.com/science/ article/pii/S0065215608701212, doi:https://doi.org/10.1016/S0065-2156(08)70121-2.

Bažant, Z.P., Belytschko, T.B., Chang, T.P., 1984. Continuum theory for strain-softening. Journal of Engineering Mechanics 110, 1666-1692.

Becker, G., Noels, L., 2012. A full-discontinuous galerkin formulation of nonlinear kirchhofflove shells: elasto-plastic finite deformations, parallel computation, and fracture applications. International Journal for Numerical Methods in Engineering 93, 80-117. URL: https://onlinelibrary.wiley.com/doi/abs/10.1002/nme.4381, doi:10.1002/nme.4381, arXiv:https://onlinelibrary.wiley.com/doi/pdf/10.1002/nme.4381.

Benzerga, A., 2002. Micromechanics of coalescence in ductile fracture. Journal of the Mechanics and Physics of Solids 50, 1331 - 1362. URL: http://www.sciencedirect.com/science/article/pii/ S0022509601001259, doi:https://doi .org/10.1016/S0022-5096(01)00125-9.

Benzerga, A., Besson, J., Pineau, A., 2004. Anisotropic ductile fracture: Part ii: theory. Acta Materialia 52, 4639 - 4650. URL: http://www.sciencedirect.com/science/article/pii/S135964540400357X, doi:https://doi.org/10.1016/j.actamat.2004.06.019.

Benzerga, A.A., Leblond, J.B., 2010. Ductile fracture by void growth to coalescence, in: Aref, H., van der Giessen, E. (Eds.), Advances in Applied Mechanics. Elsevier. volume 44 of Advances in Applied Mechanics, pp. 169 - 305. URL: http://www.sciencedirect.com/science/article/pii/S006521561044003X, doi:https : //doi .org/10.1016/S0065-2156(10)44003-X.

Benzerga, A.A., Leblond, J.B., 2014. Effective yield criterion accounting for microvoid coalescence. Journal of Applied Mechanics 81, 031009. doi:10.1115/1.4024908.

Benzerga, A.A., Leblond, J.B., Needleman, A., Tvergaard, V., 2016. Ductile failure modeling. International Journal of Fracture 201, 29-80. URL: https://doi.org/10.1007/s10704-016-0142-6, doi:10.1007/ s10704-016-0142-6.

Besson, J., 2009. Damage of ductile materials deforming under multiple plastic or viscoplastic mechanisms. International Journal of Plasticity 25, 2204 - 2221. URL: http://www.sciencedirect.com/science/ article/pii/S0749641909000357, doi:https://doi.org/10.1016/j.ijplas.2009.03.001.

Besson, J., 2010. Continuum models of ductile fracture: A review. International Journal of Damage Mechanics 19, 3-52. URL: https://doi.org/10.1177/1056789509103482, doi:10.1177/1056789509103482.

Besson, J., Steglich, D., Brocks, W., 2001. Modeling of crack growth in round bars and plane strain specimens. International Journal of Solids and Structures 38, 8259 - 8284. URL: http://www.sciencedirect.com/science/article/pii/S0020768301001676, doi:https://doi.org/ 10.1016/S0020-7683(01)00167-6.

Besson, J., Steglich, D., Brocks, W., 2003. Modeling of plane strain ductile rupture. International Journal of Plasticity 19, 1517 - 1541. URL: http://www.sciencedirect.com/science/article/pii/ S0749641902000220, doi:https://doi .org/10.1016/S0749-6419(02)00022-0.

de Borst, R., Verhoosel, C.V., 2016. Gradient damage vs phase-field approaches for fracture: Similarities and differences. Computer Methods in Applied Mechanics and Engineering 312, 78 - 94. URL: http://www.sciencedirect.com/science/article/pii/S0045782516303796, doi:https://doi.org/ 10.1016/j.cma.2016.05.015. phase Field Approaches to Fracture. 
Bosco, E., Kouznetsova, V.G., Geers, M.G.D., 2015. Multi-scale computational homogenization-localization for propagating discontinuities using $\mathrm{x}$-fem. International Journal for Numerical Methods in Engineering 102, 496-527. URL: https://onlinelibrary.wiley.com/doi/abs/10.1002/nme.4838, doi:10.1002/ nme.4838, arXiv:https://onlinelibrary.wiley.com/doi/pdf/10.1002/nme.4838.

Brocks, W., Sun, D.Z., Hönig, A., 1995. Verification of the transferability of micromechanical parameters by cell model calculations with visco-plastic materials. International Journal of Plasticity 11, 971 989. URL: http://www.sciencedirect.com/science/article/pii/S0749641995000399, doi:https: //doi.org/10.1016/S0749-6419(95)00039-9.

Brünig, M., Gerke, S., Schmidt, M., 2018. Damage and failure at negative stress triaxialities: Experiments, modeling and numerical simulations. International Journal of Plasticity 102, 70 - 82. URL: http://www.sciencedirect.com/science/article/pii/S0749641917305764, doi:https://doi.org/ 10.1016/j.ijplas.2017.12.003.

Camacho, G., Ortiz, M., 1996. Computational modelling of impact damage in brittle materials. International Journal of Solids and Structures 33, 2899 - 2938. URL: http://www.sciencedirect.com/science/ article/pii/0020768395002553, doi:https://doi.org/10.1016/0020-7683(95)00255-3.

Cazes, F., Coret, M., Combescure, A., Gravouil, A., 2009. A thermodynamic method for the construction of a cohesive law from a nonlocal damage model. International Journal of Solids and Structures 46, 1476 1490. URL: http://www.sciencedirect.com/science/article/pii/S0020768308004903, doi:https: //doi.org/10.1016/j.ijsolstr.2008.11.019.

Cazes, F., Simatos, A., Coret, M., Combescure, A., 2010. A cohesive zone model which is energetically equivalent to a gradient-enhanced coupled damage-plasticity model. European Journal of Mechanics - A/Solids 29, 976 - 989. URL: http://www.sciencedirect.com/science/article/pii/S0997753809001338, doi:https://doi.org/10.1016/j.euromechsol.2009.11.003.

Chu, C., Needleman, A., 1980. Void nucleation effects in biaxially stretched sheets. Journal of engineering materials and technology 102, 249-256.

Chung, J., Hulbert, G., 1993. A time integration algorithm for structural dynamics with improved numerical dissipation: the generalized- $\alpha$ method. Journal of applied mechanics 60, 371-375. doi:doi:10.1115/1. 2900803.

Comi, C., Mariani, S., Perego, U., 2006. An extended fe strategy for transition from continuum damage to mode i cohesive crack propagation. International Journal for Numerical and Analytical Methods in Geomechanics 31, 213-238. URL: https://onlinelibrary.wiley.com/doi/abs/10.1002/nag.537, doi:10.1002/nag.537, arXiv:https://onlinelibrary.wiley.com/doi/pdf/10.1002/nag.537.

Cortese, L., Nalli, F., Rossi, M., 2016. A nonlinear model for ductile damage accumulation under multiaxial non-proportional loading conditions. International Journal of Plasticity 85, 77 - 92. URL: http://www.sciencedirect.com/science/article/pii/S0749641916301036, doi:https://doi.org/ 10.1016/j.ijplas.2016.07.003.

Cuitino, A., Ortiz, M., 1992. A material-independent method for extending stress update algorithms from small-strain plasticity to finite plasticity with multiplicative kinematics. Engineering computations 9, 437-451.

Cuvilliez, S., Feyel, F., Lorentz, E., Michel-Ponnelle, S., 2012. A finite element approach coupling a continuous gradient damage model and a cohesive zone model within the framework of quasibrittle failure. Computer Methods in Applied Mechanics and Engineering 237-240, 244 - 259. URL: http://www.sciencedirect.com/science/article/pii/S0045782512001466, doi:https://doi.org/ 10.1016/j.cma.2012.04.019.

Dugdale, D., 1960. Yielding of steel sheets containing slits. Journal of the Mechanics and Physics of Solids 8, 100 - 104. URL: http://www.sciencedirect.com/science/article/pii/0022509660900132, doi:https://doi.org/10.1016/0022-5096(60)90013-2.

Dunand, M., Mohr, D., 2011. Optimized butterfly specimen for the fracture testing of sheet materials under combined normal and shear loading. Engineering Fracture Mechanics 78, 2919 - 2934. URL: http://www.sciencedirect.com/science/article/pii/S0013794411003067, doi:https://doi.org/ 10.1016/j.engfracmech.2011.08.008. 
Esmaeili, A., Steinmann, P., Javili, A., 2017. Non-coherent energetic interfaces accounting for degradation. Computational Mechanics 59, 361-383. URL: https://doi.org/10.1007/s00466-016-1342-7, doi:10. $1007 / \mathrm{s} 00466-016-1342-7$.

Faleskog, J., Gao, X., Shih, C.F., 1998. Cell model for nonlinear fracture analysis - i. micromechanics calibration. International Journal of Fracture 89, 355-373. URL: https ://doi .org/10.1023/A : 1007421420901, doi:10.1023/A: 1007421420901.

Forest, S., 2009. Micromorphic approach for gradient elasticity, viscoplasticity, and damage. Journal of Engineering Mechanics 135, 117-131. URL: http://ascelibrary.org/doi/ 10. 1061/\{\%\}28ASCE $\{\%\} 290733-9399\{\%\} 282009\{\%\} 29135\{\%\} 3 A 3\{\%\} 28117\{\%\} 29, \quad$ doi:10. $1061 /$ (ASCE) 0733-9399(2009) 135:3(117).

Geers, M., de Borst, R., Brekelmans, W., Peerlings, R., 1998. Strain-based transient-gradient damage model for failure analyses. Computer Methods in Applied Mechanics and Engineering 160, 133 153. URL: http://www.sciencedirect.com/science/article/pii/S004578259880011X, doi:https: //doi.org/10.1016/S0045-7825(98)80011-X.

Geuzaine, C., Remacle, J.F., 2009. Gmsh: A 3-d finite element mesh generator with built-in preand post-processing facilities. International Journal for Numerical Methods in Engineering 79, 13091331. URL: https://onlinelibrary.wiley.com/doi/abs/10.1002/nme.2579, doi:10.1002/nme.2579, arXiv:https://onlinelibrary.wiley.com/doi/pdf/10.1002/nme.2579.

Gurson, A.L., 1977. Continuum theory of ductile rupture by void nucleation and growth: Part iyield criteria and flow rules for porous ductile media. Journal of engineering materials and technology 99, 2-15. doi:10.1115/1.3443401.

Guzmán, C.F., Yuan, S., Duchne, L., Flores, E.I.S., Habraken, A.M., 2018. Damage prediction in single point incremental forming using an extended gurson model. International Journal of Solids and Structures 151, 45 - 56. URL: http://www.sciencedirect.com/science/article/pii/S0020768317301646, doi:https://doi.org/10.1016/j.ijsolstr.2017.04.013. special Issue with a Selection of Papers from the NUMISHEET 2016 Conference.

Hillerborg, A., Moder, M., Petersson, P.E., 1976. Analysis of crack formation and crack growth in concrete by means of fracture mechanics and finite elements. Cement and Concrete Research 6, 773 - 781. URL: http://www.sciencedirect.com/science/article/pii/0008884676900077, doi:https: //doi.org/10.1016/0008-8846(76)90007-7.

Huespe, A., Needleman, A., Oliver, J., Sánchez, P., 2009. A finite thickness band method for ductile fracture analysis. International Journal of Plasticity 25, 2349 - 2365. URL: http://www. sciencedirect.com/ science/article/pii/S0749641909000461, doi:https://doi.org/10.1016/j.ijplas.2009.03.005.

Huespe, A., Needleman, A., Oliver, J., Sánchez, P., 2012. A finite strain, finite band method for modeling ductile fracture. International Journal of Plasticity 28, 53 - 69. URL: http://www. sciencedirect.com/ science/article/pii/S0749641911000866, doi:https://doi.org/10.1016/j.ijplas.2011.05.010.

Johnson, G.R., Cook, W.H., 1985. Fracture characteristics of three metals subjected to various strains, strain rates, temperatures and pressures. Engineering Fracture Mechanics 21, 31-48. URL: https:// linkinghub.elsevier.com/retrieve/pii/0013794485900529, doi:10.1016/0013-7944 (85) 90052-9.

Keralavarma, S., Chockalingam, S., 2016. A criterion for void coalescence in anisotropic ductile materials. International Journal of Plasticity 82, 159 - 176. URL: http://www.sciencedirect.com/science/ article/pii/S0749641916300274, doi:https://doi.org/10.1016/j.ijplas.2016.03.003.

Keralavarma, S.M., 2017. A multi-surface plasticity model for ductile fracture simulations. Journal of the Mechanics and Physics of Solids 103, $100-120$. URL: http://www.sciencedirect.com/science/ article/pii/S0022509616307359, doi:https://doi.org/10.1016/j.jmps.2017.03.005.

Koplik, J., Needleman, A., 1988. Void growth and coalescence in porous plastic solids. International Journal of Solids and Structures 24,835-853. URL: http://www.sciencedirect.com/science/article/pii/ 0020768388900510, doi:https://doi .org/10.1016/0020-7683(88)90051-0.

Leblond, J.B., Mottet, G., 2008. A theoretical approach of strain localization within thin planar bands in porous ductile materials. Comptes Rendus Mcanique 336, 176 - 189. URL: http://www.sciencedirect.com/science/article/pii/S1631072107002215, doi:https://doi.org/ 
10.1016/j.crme.2007.11.008. duality, inverse problems and nonlinear problems in solid mechanics.

Leclerc, J., Wu, L., Nguyen, V.D., Noels, L., 2018. A damage to crack transition model accounting for stress triaxiality formulated in a hybrid nonlocal implicit discontinuous galerkin-cohesive band model framework. International Journal for Numerical Methods in Engineering 113, 374-410. URL: http: //dx.doi.org/10.1002/nme.5618, doi:10.1002/nme.5618. nme.5618.

Lemaitre, J., 1986. Local approach of fracture. Engineering Fracture Mechanics 25, 523 - 537. URL: http://www.sciencedirect.com/science/article/pii/0013794486900214, doi:https://doi. org/10.1016/0013-7944(86)90021-4.

Linder, C., Armero, F., 2007. Finite elements with embedded strong discontinuities for the modeling of failure in solids. International Journal for Numerical Methods in Engineering 72, 13911433. URL: https://onlinelibrary.wiley.com/doi/abs/10.1002/nme.2042, doi:10.1002/nme.2042, arXiv:https://onlinelibrary.wiley.com/doi/pdf/10.1002/nme.2042.

Liu, Z., Wong, W., Guo, T., 2016. Void behaviors from low to high triaxialities: Transition from void collapse to void coalescence. International Journal of Plasticity 84, 183 - 202. URL: http: //www. sciencedirect. com/science/article/pii/S0749641916300821, doi:https://doi.org/10.1016/j.ijplas.2016.05. 008.

Mediavilla, J., Peerlings, R.H.J., Geers, M.G.D., 2005. Discrete crack modelling of ductile fracture driven by non-local softening plasticity. International Journal for Numerical Methods in Engineering 66, 661688. URL: https://onlinelibrary.wiley.com/doi/abs/10.1002/nme.1572, doi:10.1002/nme.1572, arXiv:https://onlinelibrary.wiley.com/doi/pdf/10.1002/nme.1572.

Mergheim, J., Kuhl, E., Steinmann, P., 2004. A hybrid discontinuous galerkin/interface method for the computational modelling of failure. Communications in Numerical Methods in Engineering 20, 511519. URL: https://onlinelibrary.wiley.com/doi/abs/10.1002/cnm.689, doi:10.1002/cnm.689, arXiv:https://onlinelibrary.wiley.com/doi/pdf/10.1002/cnm. 689.

Miehe, C., Aldakheel, F., Raina, A., 2016a. Phase field modeling of ductile fracture at finite strains: A variational gradient-extended plasticity-damage theory. International Journal of Plasticity 84, 1 32. URL: http://www.sciencedirect.com/science/article/pii/S0749641916300602, doi:https:// doi.org/10.1016/j.ijplas.2016.04.011.

Miehe, C., Kienle, D., Aldakheel, F., Teichtmeister, S., 2016b. Phase field modeling of fracture in porous plasticity: A variational gradient-extended Eulerian framework for the macroscopic analysis of ductile failure. Computer Methods in Applied Mechanics and Engineering 312, 3-50. URL: https: //linkinghub.elsevier.com/retrieve/pii/S0045782516305412, doi:10.1016/j.cma.2016.09.028.

Moës, N., Dolbow, J., Belytschko, T., 1999. A finite element method for crack growth without remeshing. International Journal for Numerical Methods in Engineering 46, 131-150.

Mota, A., Knap, J., Ortiz, M., 2008. Fracture and fragmentation of simplicial finite element meshes using graphs. International Journal for Numerical Methods in Engineering 73, 1547-1570. URL: http://dx . doi.org/10.1002/nme.2135.

Nahshon, K., Hutchinson, J., 2008. Modification of the gurson model for shear failure. European Journal of Mechanics - A/Solids 27, 1 - 17. URL: http://www.sciencedirect.com/science/article/pii/ S0997753807000721, doi:http://dx.doi.org/10.1016/j.euromechsol .2007.08.002.

Nguyen, G.D., Korsunsky, A.M., Belnoue, J.P.H., 2015. A nonlocal coupled damage-plasticity model for the analysis of ductile failure. International Journal of Plasticity 64, 56 - 75. URL: http://www.sciencedirect.com/science/article/pii/S0749641914001491, doi:https://doi.org/ 10.1016/j.ijplas.2014.08.001.

Nguyen, V.D., Pardoen, T., Noels, L., Submitted. An implicit nonlocal approach of ductile failure incorporating void growth, internal necking coalescence, and shear coalescence mechanisms. Journal of the Mechanics and Physics of Solids .

Nielsen, K.L., Tvergaard, V., 2010. Ductile shear failure or plug failure of spot welds modelled by modified gurson model. Engineering Fracture Mechanics 77, 1031 - $1047 . \quad$ URL: http://www.sciencedirect.com/science/article/pii/S0013794410001128, doi:https://doi.org/ $10.1016 / j$.engfracmech.2010.02.031. 
Noels, L., Radovitzky, R., 2006. A general discontinuous galerkin method for finite hyperelasticity. formulation and numerical applications. International Journal for Numerical Methods in Engineering 68, 6497. URL: https://onlinelibrary.wiley.com/doi/abs/10.1002/nme.1699, doi:10.1002/nme.1699, arXiv:https://onlinelibrary.wiley.com/doi/pdf/10.1002/nme.1699.

Pardoen, T., Hutchinson, J., 2000. An extended model for void growth and coalescence. Journal of the Mechanics and Physics of Solids 48, 2467 - 2512. URL: http://www.sciencedirect.com/science/ article/pii/S0022509600000193, doi:https://doi.org/10.1016/S0022-5096(00)00019-3.

Pardoen, T., Scheyvaerts, F., Simar, A., Tekoğlu, C., Onck, P.R., 2010. Multiscale modeling of ductile failure in metallic alloys. Comptes Rendus Physique 11, $326-345$. URL: http://www.sciencedirect.com/science/article/pii/S1631070510000770, doi:https://doi.org/ 10.1016/j.crhy.2010.07.012. computational metallurgy and scale transitions.

Paulino, G., Celes, W., Espinha, R., Zhang, Z., 2008. A general topology-based framework for adaptive insertion of cohesive elements in finite element meshes. Engineering with Computers 24, 59-78. URL: http://dx.doi.org/10.1007/s00366-007-0069-7. 10.1007/s00366-007-0069-7.

Peerlings, R., Geers, M., de Borst, R., Brekelmans, W., 2001. A critical comparison of nonlocal and gradient-enhanced softening continua. International Journal of Solids and Structures 38, 7723 7746. URL: http://www.sciencedirect.com/science/article/pii/S0020768301000877, doi:https: //doi.org/10.1016/S0020-7683(01)00087-7.

Peerlings, R.H.J., de Borst, R., Brekelmans, W.A.M., De Vree, J.H.P., 1996. Gradient enhanced damage for quasi-brittle materials. International Journal for Numerical Methods in Engineering 39, 3391-3403. doi:10.1002/(SICI) 1097-0207(19961015) 39:19<3391: :AID-NME7>3 . 0 . C0;2-D.

Pineau, A., Benzerga, A., Pardoen, T., 2016. Failure of metals i: Brittle and ductile fracture. Acta Materialia 107, 424 - 483. URL: http://www.sciencedirect.com/science/article/pii/S1359645415301403, doi:https://doi.org/10.1016/j.actamat.2015.12.034.

Poh, L.H., Sun, G., 2016. Localizing gradient damage model with decreasing interactions. International Journal for Numerical Methods in Engineering 110, 503-522. URL: https://onlinelibrary.wiley.com/doi/abs/10.1002/nme.5364, doi:10.1002/nme.5364, arXiv:https://onlinelibrary.wiley.com/doi/pdf/10.1002/nme.5364.

Prechtel, M., Leugering, G., Steinmann, P., Stingl, M., 2011. Towards optimization of crack resistance of composite materials by adjustment of fiber shapes. Engineering Fracture Mechanics 78, $944-$ 960. URL: http://www.sciencedirect.com/science/article/pii/S0013794411000117, doi:https: //doi.org/10.1016/j.engfracmech.2011.01.007.

Radovitzky, R., Seagraves, A., Tupek, M., Noels, L., 2011. A scalable 3d fracture and fragmentation algorithm based on a hybrid, discontinuous galerkin, cohesive element method. Computer Methods in Applied Mechanics and Engineering 200, 326 - 344. URL: http://www.sciencedirect.com/science/ article/pii/S0045782510002471, doi:https://doi.org/10.1016/j.cma.2010.08.014.

Reddi, D., Areej, V., Keralavarma, S., 2019. Ductile failure simulations using a multi-surface coupled damage-plasticity model. International Journal of Plasticity 118, 190 - 214 . URL: http://www.sciencedirect.com/science/article/pii/S0749641918307149, doi:https://doi.org/ 10.1016/j.ijplas.2019.02.007.

Remmers, J.J.C., de Borst, R., Verhoosel, C.V., Needleman, A., 2013. The cohesive band model: a cohesive surface formulation with stress triaxiality. International Journal of Fracture 181, 177-188. URL: https: //doi.org/10.1007/s10704-013-9834-3, doi:10.1007/s10704-013-9834-3.

Reusch, F., Svendsen, B., Klingbeil, D., 2003a. Local and non-local gurson-based ductile damage and failure modelling at large deformation. European Journal of Mechanics - A/Solids 22, 779 792. URL: http://www.sciencedirect.com/science/article/pii/S0997753803000706, doi:https: //doi.org/10.1016/S0997-7538(03)00070-6.

Reusch, F., Svendsen, B., Klingbeil, D., 2003b. A non-local extension of gurson-based ductile damage modeling. Computational Materials Science 26, 219 - 229. URL: http://www.sciencedirect.com/ science/article/pii/S0927025602004020, doi:https://doi.org/10.1016/S0927-0256(02) 00402-0. 11th International Workshop on Computational Mechanics of Materials. 
Roth, C.C., Morgeneyer, T.F., Cheng, Y., Helfen, L., Mohr, D., 2018. Ductile damage mechanism under shear-dominated loading: In-situ tomography experiments on dual phase steel and localization analysis. International Journal of Plasticity 109, 169 - 192. URL: http://www.sciencedirect.com/science/ article/pii/S0749641917306940, doi:https://doi.org/10.1016/j.ijplas.2018.06.003.

Scheyvaerts, F., Pardoen, T., Onck, P., 2010. A new model for void coalescence by internal necking. International Journal of Damage Mechanics 19, 95-126. doi:10.1177/1056789508101918.

Shen, R., Waisman, H., Guo, L., 2019. Fracture of viscoelastic solids modeled with a modified phase field method. Computer Methods in Applied Mechanics and Engineering 346, 862-890. URL: http://www.sciencedirect.com/science/article/pii/S0045782518304699, doi:https://doi.org/ 10.1016/j.cma.2018.09.018.

Siegmund, T., Brocks, W., 2000. A numerical study on the correlation between the work of separation and the dissipation rate in ductile fracture. Engineering Fracture Mechanics 67, 139 154. URL: http://www.sciencedirect.com/science/article/pii/S0013794400000540, doi:https: //doi.org/10.1016/S0013-7944(00)00054-0.

Tekoğlu, C., Hutchinson, J.W., Pardoen, T., 2015. On localization and void coalescence as a precursor to ductile fracture. Philosophical Transactions of the Royal Society A: Mathematical, Physical and Engineering Sciences 373, 20140121. URL: https: //royalsocietypublishing.org/doi/abs/10.1098/rsta.2014.0121, doi:10.1098/rsta.2014.0121, arXiv:https://royalsocietypublishing.org/doi/pdf/10.1098/rsta.2014.0121.

Tekoğlu, C., Leblond, J.B., Pardoen, T., 2012. A criterion for the onset of void coalescence under combined tension and shear. Journal of the Mechanics and Physics of Solids 60, 1363 - 1381. URL: http://www.sciencedirect.com/science/article/pii/S0022509612000373, doi:https://doi.org/ $10.1016 / j \cdot j m p s .2012 .02 .006$.

Thomason, P., 1985a. A three-dimensional model for ductile fracture by the growth and coalescence of microvoids. Acta Metallurgica 33, 1087 - 1095. URL: http://www. sciencedirect.com/science/article/ pii/0001616085902020, doi:https://doi .org/10.1016/0001-6160(85)90202-0.

Thomason, P., 1985b. Three-dimensional models for the plastic limit-loads at incipient failure of the intervoid matrix in ductile porous solids. Acta Metallurgica 33, 1079 - 1085. URL: http:// www.sciencedirect.com/science/article/pii/0001616085902019, doi:https://doi.org/10.1016/ 0001-6160 (85) 90201-9.

Torki, M., Tekoglu, C., Leblond, J.B., Benzerga, A., 2017. Theoretical and numerical analysis of void coalescence in porous ductile solids under arbitrary loadings. International Journal of Plasticity 91, 160 - 181. URL: http://www.sciencedirect.com/science/article/pii/S0749641916302595, doi:https: //doi.org/10.1016/j.ijplas.2017.02.011.

Torki, M.E., 2019. A unified criterion for void growth and coalescence under combined tension and shear. International Journal of Plasticity 119, 57 - 84. URL: http://www.sciencedirect.com/science/ article/pii/S0749641918303152, doi:https://doi.org/10.1016/j.ijplas.2019.02.002.

Tvergaard, V., 1981. Influence of voids on shear band instabilities under plane strain conditions. International Journal of Fracture 17, 389-407. URL: https://doi.org/10.1007/BF00036191, doi:10.1007/ BF00036191.

Tvergaard, V., Needleman, A., 1984. Analysis of the cup-cone fracture in a round tensile bar. Acta Metallurgica 32, 157 - 169. URL: http://www.sciencedirect.com/science/article/pii/000161608490213X, doi:https://doi.org/10.1016/0001-6160(84)90213-X.

Vandoren, B., Simone, A., 2018. Modeling and simulation of quasi-brittle failure with continuous anisotropic stress-based gradient-enhanced damage models. Computer Methods in Applied Mechanics and Engineering 332, 644-685. URL: http://www.sciencedirect.com/science/article/pii/S0045782517307776, doi:https://doi.org/10.1016/j.cma.2017.12.027.

Wang, Y., Waisman, H., 2018. An arc-length method for controlled cohesive crack propagation using high-order xfem and irwins crack closure integral. Engineering Fracture Mechanics 199, 235 256. URL: http://www.sciencedirect.com/science/article/pii/S0013794418301358, doi:https: //doi.org/10.1016/j.engfracmech.2018.05.018. 
Wu, L., Becker, G., Noels, L., 2014. Elastic damage to crack transition in a coupled non-local implicit discontinuous galerkin/extrinsic cohesive law framework. Computer Methods in Applied Mechanics and Engineering 279, 379 - 409. URL: http://www.sciencedirect.com/science/article/pii/ S0045782514002175, doi:https://doi.org/10.1016/j.cma.2014.06.031.

Wu, L., Tjahjanto, D., Becker, G., Makradi, A., Jrusalem, A., Noels, L., 2013. A micromeso-model of intralaminar fracture in fiber-reinforced composites based on a discontinuous galerkin/cohesive zone method. Engineering Fracture Mechanics 104, 162 - 183. URL: http://www.sciencedirect.com/science/ article/pii/S0013794413001252, doi:https://doi.org/10.1016/j. engfracmech.2013.03.018.

Xu, Q., Lu, Z., 2013. An elasticplastic cohesive zone model for metalceramic interfaces at finite deformations. International Journal of Plasticity 41, 147 - 164. URL: http://www.sciencedirect.com/science/ article/pii/S0749641912001386, doi:https://doi.org/10.1016/j.ijplas.2012.09.008.

Xue, Z., Pontin, M., Zok, F., Hutchinson, J., 2010. Calibration procedures for a computational model of ductile fracture. Engineering Fracture Mechanics 77, 492-509. URL: https://linkinghub.elsevier. com/retrieve/pii/S0013794409003233, doi:10.1016/j. engfracmech.2009.10.007.

Zhang, Y., Lorentz, E., Besson, J., 2018. Ductile damage modelling with locking-free regularised gtn model. International Journal for Numerical Methods in Engineering 113, 18711903. URL: https://onlinelibrary.wiley.com/doi/abs/10.1002/nme.5722, doi:10.1002/nme.5722, arXiv:https://onlinelibrary.wiley.com/doi/pdf/10.1002/nme.5722.

Zhang, Z., Thaulow, C., Odegard, J., 2000. A complete gurson model approach for ductile fracture. Engineering Fracture Mechanics 67, 155 - 168. URL: http://www.sciencedirect.com/science/article/ pii/S0013794400000552, doi:https://doi.org/10.1016/S0013-7944(00)00055-2. 